\title{
A quantitative NLTE analysis of visual and ultraviolet spectra of four helium-rich subdwarf $O$ stars
}

\author{
M. Schindewolf ${ }^{1}$, P. Németh ${ }^{1,2,3}$, U. Heber ${ }^{1}$, T. Battich ${ }^{4,5}$, M. M. Miller Bertolami ${ }^{4}$, A. Irrgang ${ }^{1}$, and M. Latour ${ }^{1,6}$ \\ ${ }^{1}$ Dr. Karl Remeis Observatory, University of Erlangen-Nuremberg, Sternwartstr. 7, 96049 Bamberg, Germany \\ e-mail: ulrich.heber@fau.de \\ 2 Astronomical Institute of the Czech Academy of Sciences, 25165 Ondřejov, Czech Republic \\ 3 Astroserver.org, 8533 Malomsok, Hungary \\ 4 Instituto de Astrofísica de La Plata, CONICET-UNLP, Argentina \\ ${ }^{5}$ Facultad de Ciencias Astronómicas y Geofísicas, UNLP, Argentina \\ ${ }^{6}$ Institut für Astrophysik, Georg-August-Universität, Göttingen, Germany
}

Received 20 October 2017 / Accepted 20 August 2018

\begin{abstract}
Aims. Hot subdwarf stars represent a poorly understood late phase of stellar evolution. While binary evolution plays an important role for the formation of B-type subdwarfs ( $\mathrm{sdB}$ ), the origin of the helium dominated subclass of O-type subdwarfs (He-sdO) is still unknown. We search for chemical signatures of their genesis by means of quantitative spectral analyses of high-quality visual and ultraviolet spectra.

Methods. Four prototypical He-sdO stars, one belonging to the nitrogen-rich and three to the C-rich subclass, were selected for which archival far-ultraviolet spectra from the FUSE satellite as well as high-resolution visual and UVA spectra taken with the ESO-UVES/FEROS spectrographs are available. Using TLUSTY200/SynSPEC49 to compute line blanketed- non-local thermodynamic equilibrium (NLTE) model atmospheres and synthetic spectra, atmospheric parameters and the abundances patterns have been derived. The final models included $\mathrm{H}, \mathrm{He}, \mathrm{C}, \mathrm{N}, \mathrm{O}, \mathrm{Ne}, \mathrm{Mg}, \mathrm{Al}, \mathrm{Si}, \mathrm{P}, \mathrm{S}, \mathrm{Fe}$, and $\mathrm{Ni}$ represented by the most detailed model atoms available. Because of the enrichment of either nitrogen or carbon, it turned out, that models including these elements at the appropriate high abundance provide sufficiently accurate approximations to the temperature stratification of full models.

Results. No indications for binarity were found, neither radial velocity variations nor photometric evidence for the presence of a companion could be detected. All stars have helium-dominated atmospheres almost free of hydrogen and temperatures between $42000 \mathrm{~K}$ and $47000 \mathrm{~K}$ while their surface gravities lie between $\log g=5.4$ and 5.7. The abundance pattern of CD-31 4800 displays the signatures of $\mathrm{CNO}$ burning, while heavier elements are subsolar by about 0.4 dex, except for $\mathrm{Ne}$ and $\mathrm{Si}$ which are close to solar. The abundance patterns of the C-rich He-sdOs are more complex. A slightly subsolar metallicity is accompanied by $\mathrm{N}$-enrichment and O-deficiency, less pronounced than in $\mathrm{CD}-31^{\circ} 4800$. Neon is mildly to strongly enriched, up to a factor of ten with respect to the sun in LS IV $+10^{\circ}$ 9. The nickel-to-iron ratio is significantly super-solar. Using spectral energy distributions and Gaia parallaxes the masses of the stars were determined. They are found to scatter around the canonical mass for the core helium flash, although the uncertainties are large.

Conclusions. The abundance pattern observed for CD-31 4800 is consistent with predictions of models for slow (cold) mergers of pairs of equal mass helium WDs except for the low oxygen abundance observed. Models for composite mergers were considered for the C-rich stars, but predict abundance pattern dissimilar to those determined. [CW83] 0904-02, though, may be a candidate for a composite He-WD merger, as it rotates and appears to be more massive than the other program stars. New evolutionary models for the hot flasher scenario predict abundance patterns similar to those determined for the C-rich stars. Hence, C-rich He-sdO may well result from late He flashes with deep-mixing episodes.
\end{abstract}

Key words. stars: abundances - stars: atmospheres - stars: evolution - subdwarfs

\section{Introduction}

Hot subluminous stars $(\mathrm{sdB}$ and $\mathrm{sdO})$ represent late stages of the evolution of low-mass stars and can be found between the main sequence and the white dwarf (WD) sequence in the Hertzsprung-Russell diagram. Most hot subdwarf stars, in particular the sdB stars, are core helium-burning stars with very thin hydrogen envelopes. Hence, the sdB stars populate the extreme horizontal branch (EHB). Unlike normal horizontal branch stars EHB stars are unable to sustain hydrogen shell burning (Heber 2009) and evolve directly to the WD cooling sequence without an excursion to the AGB.

They can be found both in the old disk and the halo of the Galaxy (Ferraro et al. 1997; Napiwotzki 2008) and are sufficiently abundant to dominate the population of faint blue stars at high Galactic latitudes to about eighteens magnitude. Hot subdwarf stars are believed to be the dominating source for the UV upturn phenomenon that can be observed in elliptical galaxies and galaxy bulges (Brown et al. 1997; Han et al. 2007).

While sdB stars form a rather homogeneous class of stars, subluminous $\mathrm{O}$ stars have more diverse stellar and atmospheric properties. Luminosities range from a few tens of solar to some thousands and effective temperatures of sdO stars span a large range from $40000 \mathrm{~K}$ to $100000 \mathrm{~K}$ (Napiwotzki 2008; Heber 2009, 2016). The most luminous ones, however, are believed to be post-AGB stars similar to central stars of planetary nebulae. The atmospheric composition of sdO stars is diverse as well, in particular with respect to the abundances of helium, 
carbon, and nitrogen. In hydrogen dominated sdO atmospheres the helium content may be subsolar while helium is dominating over hydrogen in the He-sdO subclass. Hydrogen is hardly detectable for some of those stars. The origin of these He-sdOs is ambiguous as they are believed to form differently from the hydrogen-dominated sdO stars, which are naturally explained as the progeny of the sdB stars in a post-EHB stage of evolution. The low fraction of close binaries among He-sdO stars (Napiwotzki 2008) suggests that they may originate from the merger of two helium WDs (Webbink 1984). However, this scenario is rivaled by the so called late hot flasher scenario, which does not implicate binary evolution. Instead, the helium, carbon, and/or nitrogen enrichments are attributed to internal flash phenomena occurring when a star has already embarked on the WD cooling sequence (Sweigart 1997). Interest in the hot flasher scenario revived, when the small group of helium rich sdB stars attracted attention (Ahmad \& Jeffery 2003). Naslim et al. (2010) suggested that two subclasses should be distinguished according to their helium content: the intermediate iHe-sdBs with heliumto-hydrogen ratios between solar and $\mathrm{He} / \mathrm{H}=4$ (by number), because He-sdBs with higher helium abundance (extreme HesdBs) have surface compositions distinctively different from the very peculiar composition of iHe-sdBs. The results of the spectral analyses of hot subdwarf stars from the ESO-SPY project (Lisker et al. 2005; Stroeer et al. 2007) suggest that such a distinction may be useful for the He-sdOs as well (see Heber 2016, for a discussion). Therefore, we shall restrict the term He$\mathrm{sdO}$ to those with almost pure helium line spectra (hydrogen Balmer components too weak to significantly contribute to the equivalent widths of the $\mathrm{He} \mathrm{II} / \mathrm{H}$ I blends) throughout this paper. While spectral analyses of many subluminous B stars are available (e.g. Edelmann et al. 2003; Lisker et al. 2005; Németh et al. 2012a; Geier 2013; Fontaine et al. 2014), the sdO stars have not been studied extensively. This is partly caused by the need of model atmospheres in non-Local Thermodynamic Equilibrium (NLTE). When such atmospheres became available, first quantitative analyses were carried out from low (e.g. Dreizler et al. 1990) as well as high-resolution spectra (e.g. Dreizler 1993). Németh et al. (2012b) have presented a NLTE analysis from low resolution optical spectra of a magnitude limited subdwarf sample from the solar neighborhood, including $124 \mathrm{sdB}$ and $42 \mathrm{sdO}$ stars. Detailed abundance analyses, however, remained scarce.

The detailed abundance pattern of He-sdO stars are important to test the formation scenarios. The latest generation of NLTE model atmospheres is now able to treat NLTE and line blanketing effects by heavy elements consistently and allow abundances of low, intermediate and higher mass elements (iron, nickel) to be determined. In recent years several quantitative spectral analyses have been carried out, making use of visual spectra of low (e.g. Németh et al. 2012b) as well as high resolution (Hirsch 2009). Those analyses, however, were restricted to the chemical elements H, He, C \& N (Hirsch 2009) and H, He, C, N \& O (Németh et al. 2012b), only. Accordingly, HesdO stars may be grouped into three subclasses, according to their carbon and/or nitrogen line strength; the C strong-lined, the $\mathrm{N}$ strong-lined and those where both carbon and nitrogen lines being strong (CN, Stroeer et al. 2007). Actually, the $\mathrm{CN}$ subclass is not well defined, because the classification scheme by Stroeer et al. (2007) is based on spectra of different signal-tonoise ratio $(\mathrm{S} / \mathrm{N})$, most being much lower than the spectra presented here. Therefore, we distinguish $\mathrm{N}$ strong-lined (N-type) from $\mathrm{C}$ strong-lined (C-type) stars, only. This calls for more detailed abundances studies.

Visual Echelle spectra of excellent quality (high spectral resolution and $\mathrm{S} / \mathrm{N}$, as well as broad wavelength range) have become available and the FUSE mission has opened up the far-ultraviolet (FUV) spectral window to the Lyman series limit. FUSE spectra of high spectral resolution and $\mathrm{S} / \mathrm{N}$ are now at hand superseding IUE UV spectra previously available for some $\mathrm{He}$-sdOs both in terms of $\mathrm{S} / \mathrm{N}$ and spectral resolution and allowing access to a larger number of elements than from visual spectra. Hot subdwarf stars have been observed in the far UV with the aim to probe the interstellar medium, in particular by making use of the FUSE satellite (e.g. Sonneborn et al. 2002; Friedman et al. 2002; Jenkins 2013). Similarly, high-resolution spectra in the UVA (3200-3900 $\AA$ ) range have been taken to determine elemental abundances in the interstellar medium (e.g. Ti II, Lallement et al. 2008).

Most of the available quantitative spectral analyses of the photospheric FUV spectra have targeted the hydrogen-rich sdB and sdO stars (Ohl et al. 2000; Blanchette et al. 2008; Fontaine et al. 2006; Chayer et al. 2006; Latour et al. 2013, 2018) and three helium-rich sdB stars (Lanz et al. 2004). To our knowledge no He-sdO has been analyzed from FUSE or UVA spectra. Hence, we considered it worthwhile to revisit some of the brightest He-sdO stars, for which data from modern groundbased Echelle spectrographs as well as from the FUSE satellite are at hand. We judged four stars to be the best suited ones, from both subclasses of He-sdOs, CD-31 4800 (N strong-lined), LSS 1274, and [CW83] 0904-02, and LS IV +10 9 (C stronglined). All of them have been analyzed before using an early generation of NLTE model atmospheres. We consider these stars as benchmarks for the abundance patterns of He-sdO stars to test the predictions of different evolutionary scenarios. In addition, they provide a benchmark for NLTE model atmosphere diagnostics. Hence, we also embarked on a detailed investigation of NLTE model atmospheres of different degree of sophistication and a reanalysis of previously analyzed spectra to identify potential systematic differences.

We begin with a description of the available spectra and discuss radial velocity variability in Sect. 2 . In Sect. 3 we present our atmospheric models and spectral synthesis and provide a comparison of models of different degree of sophistication. In Sect. 4 we describe our approach to analyze visual and UVA Echelle and FUSE spectra. Atmospheric parameters are derived from visual spectroscopy in Sect. 5 along with stellar masses from spectral energy distributions and Gaia parallaxes in Sect. 6. The analysis of the metal abundances follows in Sect. 7 and results are discussed in the context of rivalling scenarios in Sect. 8. The paper ends with a brief summary and conclusions in Sect. 9.

\section{The sample and spectral observations}

CD-31 4800 was discovered by Garrison \& Hiltner (1973) to be an extremely helium rich subluminous O star. Walker (1981) found the visual spectrum of LS IV $+10^{\circ} 9$ to be similar to that of CD- $31^{\circ} 4800$ and concluded that it must be of the same spectral type as CD- $31^{\circ} 4800$. Berger \& Fringant (1980) observed low resolution UV spectra from the TD1 satellite and from a comparison with other sdO stars (including CD-31 ${ }^{\circ} 4800$ ) concluded that [CW83] 0904-02 is a sdO star similar to CD-31 ${ }^{\circ} 4800$. LSS 1274 was first described as a sdO star by Drilling \& Heber (1987). The first quantitative spectral analysis of visual spectra of CD$31^{\circ} 4800$ was carried out by Giddings (1981). With the advent of the IUE satellite high resolution ultraviolet spectra became available and early Echelle spectrographs for the visual wavelength range, such as CASPEC at the ESO 3.6m telescope, allowed high resolution spectra of sdO stars to be observed. Such visual and UV spectra were analyzed by Dreizler (1993) using the TMAP 
NLTE code (Werner \& Dreizler 1999) and by Bauer \& Husfeld (1995) using the NLTE code described by Husfeld et al. (1989) who derived atmospheric parameters and the abundance of light elements using NLTE model atmospheres and spectral synthesis. Hirsch (2009) derived H, He, C and N abundances from highresolution visual spectra using TMAP NLTE model atmospheres and spectrum synthesis (Werner \& Dreizler 1999).

FUSE spectra were used to determine the deuterium-tooxygen and the deuterium-to-hydrogen ratios in the interstellar (ISM) medium toward CD-31 4800 and LSS 1274 (Hébrard \& Moos 2003; Wood et al. 2004). Jenkins (2013) used FUSE spectra of CD $-31^{\circ} 4800$ and LS IV $+10^{\circ} 9$ and [CW83] 0904-02 to study the respective line-of-sight warm ISM. Also the hot ISM has been investigated using FUSE spectroscopy of hot subdwarf stars to search for diffuse O vi emission (Dixon et al. 2006; Blair et al. 2009). Hence, for the ISM studies the stars just provided background sources. Here we turn the analysis around and re-use the observations to analyze the stellar photospheric spectra.

\subsection{Spectroscopic observations}

For the four He-sdO stars (CD-31 4800, [CW83] 0904-02, LSS 1274 and LS IV +10 9) under investigation both visual and FUV spectra are available. The space-based FUV spectra from FUSE were obtained from the Mikulski Archive for Space Telescopes (MAST). The FUSE spectrograph covers the far UV wavelength range from 905 to $1185 \AA$ at a spectral resolution of $R=20000$. The design of the instrument and the spectroscopic data products are described by Sahnow et al. (2000), Moos et al. (2000), and Dixon et al. (2007). In addition, visual spectra, obtained with the UVES and FEROS spectrographs, were downloaded from the European Southern Observatory (ESO) Data Archive. The FEROS spectrograph covers the spectral range from 3530 to $9200 \AA$ at a resolution of $R \sim 48000$ (Kaufer et al. 1999), whereas the UVES spectrograph allow spectra with different instrument setups (Dekker et al. 2000) to be taken, resulting in different spectral coverages and resolutions.

Table A.1 gives an overview of our sample and the properties of the available spectral observations. Visual spectra taken with the CASPEC spectrograph at the ESO $3.6 \mathrm{~m}$ telescope were also available (see Dreizler 1993) in reduced and normalized form. Those spectra complement the UVES spectra of LSS 1274 and LS IV $+10^{\circ} 9$ to cover the full visual spectral range. We take the opportunity to reanalyze the CASPEC spectra and compare the results to the published ones in Sect. A.4 in the appendix. The UVES spectrum of CD-31 4800 has been observed with the image slicer to enhance the spectral resolution to about $R=$ 70000 in the blue and $R=100000$ in the red channel, respectively. Because this is a non-standard mode of observing, the archive data showed serious data reduction artifacts. S. Moehler kindly re-reduced those spectra. Despite of her extensive efforts some parts of the spectrum show residual fringing in the red part, which, however, does not compromise the spectral analysis. In addition to the visual and FUV range, we can make use of the extreme blue coverage of UVES in the wavelength range between $3200 \AA$ and about $3800 \AA$ (the UVA spectral range).

\subsection{Line identification and selection}

Line identification was done by consulting the NIST database (Kramida et al. 1989) as well as evaluating synthetic spectra calculated with the SYNSPEC code (Hubeny et al. 1994).
Especially the UVES spectrograph allows for high resolution spectra in the visual region to the atmospheric cut-off (the UVA range), which is important because it covers the limits of the hydrogen Balmer as well of helium line series. Most importantly numerous lines of light metals, in particular from $\mathrm{N}$ III, O III, and Ne II are present. For the abundance analysis we had to select spectral lines in the first place that are not blended with lines of other chemical elements nor with interstellar or telluric lines. Because the metals are added to the models subsequently (see Sect. 4), we are also able to treat line blends when the abundance of the blending element has been determined beforehand.

\subsubsection{The visual spectral range}

Spectral lines in the visual selected for the spectral analysis are listed in Tables A.2 and A.4. Besides hydrogen and helium, lines from two ions of carbon $\left(\mathrm{C}_{\mathrm{III}} \text { and } \mathrm{C} \text { IV }\right)^{1}$, three ions of nitrogen (N II, N III, and N IV) were considered. Two ions of oxygen (O II and $\mathrm{O}_{\mathrm{III}}$ ), silicon (Si III and Si IV), and phosphorus (P IV and $\mathrm{PV}$ line), and two stages of sulfur (S III, S Iv) were also used. In addition spectral lines of $\mathrm{Ne}$ II, $\mathrm{Mg}$ II and $\mathrm{Al}$ III were used as well. No spectral lines from iron group elements were identified.

\subsubsection{The FUV spectral range}

Many atomic and molecular interstellar lines are present in the FUV. Most of the atomic lines arise from neutral atoms, such as $\mathrm{N}_{\mathrm{I}}, \mathrm{O}$ I, and Ar I (see Fig. 1 of Jenkins 2013, for an example, [CW83] 0904-02). We used the table of Morton (2003) to identify the atomic interstellar lines. More difficult are the molecular interstellar lines, most prominently the Lyman and Werner bands of $\mathrm{H}_{2}$. We used the list of Morton \& Dinerstein (1976) for identification. While the Lyman and Werner lines are weak in CD$31^{\circ} 4800$ and [CW83] 0904-02, they provide more severe line confusion for LSS 1274 and LS IV $+10^{\circ} 9$.

The most obvious difference of the far-ultraviolet spectrum to the visual one is the large amount of lines of iron group elements. We concentrate on iron (Fe III, Fe IV, and Fe v) and nickel (Ni IV and Ni v). Since the lines are heavily blended, great care has to be taken to chose appropriate spectral lines. As found for other sdO stars many spectral lines remained unidentified (e.g. Friedman et al. 2002). Rauch et al. (2014) pointed out, that opacity is missing even when spectral lines from additional iron group elements are included in the calculation.

For the lighter elements (C to $\mathrm{S}$ ) the same ions as identified in the visual are accessible in the FUSE spectral range as well with the exception of magnesium. In addition, $\mathrm{O}_{\text {IV }}, \mathrm{S}_{\mathrm{V}}$ and $\mathrm{S}$ VI lines are present.

\subsubsection{Radial velocities}

The observations were shifted to the rest wavelength before the analysis of atmospheric parameters was started. To this end we determined the radial velocity of the targets for each spectrum. This also allows us to co-add different single spectra and, thus to increase the $\mathrm{S} / \mathrm{N}$. The radial velocity was measured by fitting a Voigt line profile to selected sharp metal lines. In the FUV, this was often not possible due to line blending and the lower spectral resolution. In these cases a synthetic spectrum was shifted manually and the best fit was determined by eye. Table 1 gives an overview on the measured velocities. Results from FUSE have to be dealt with caution as the wavelength calibration turned out

1 For $\mathrm{C}_{\text {II, }} 4267 \AA$ see Sects. 7.1.1 and A.5. 
to be non-consistent for some spectra. Dixon et al. (2007) found a systematic error of up to $10 \mathrm{~km} \mathrm{~s}^{-1}$ for the wavelength calibration of FUSE data, depending on the angle under which light hits the optics. We added this systematic error to the statistical ones.

Few radial velocities are reported in the literature. For CD$31^{\circ} 4800$ Giddings (1981) and Drilling \& Heber (1987) measure consistently $36 \pm 2 \pm 7 \mathrm{~km} \mathrm{~s}^{-1}$ (statistical and systematic uncertainties) and $37 \pm 1 \mathrm{~km} \mathrm{~s}^{-1}$, while Kilkenny \& Muller (1989) give $18 \pm 4 \mathrm{~km} \mathrm{~s}^{-1}$. Hirsch (2009) find the radial velocity of CD$31^{\circ} 4800$ to be constant at $36 \pm 4 \mathrm{~km} \mathrm{~s}^{-1}$ in four consecutive nights and the same 174 days later. It is therefore likely that the Kilkenny \& Muller (1989) as well as our measurements from FUSE spectra are less accurate than estimated, especially when taking the propagated error for FUSE from Dixon et al. (2007) into account. We conclude that there is no evidence for RV variations in CD-3 $1^{\circ} 4800$.

For [CW83] 0904-02 Hirsch (2009) reported radial velocities of $20 \pm 4 \mathrm{~km} \mathrm{~s}^{-1}$ and $22 \pm 3 \mathrm{~km} \mathrm{~s}^{-1}$ measured during a single night, which is consistent with $v_{\text {rad }}=24 \pm 1 \mathrm{~km} \mathrm{~s}^{-1}$ reported by Drilling \& Heber (1987). For LSS 1274 Drilling \& Heber (1987) give $v_{\text {rad }}=24 \pm 1 \mathrm{~km} \mathrm{~s}^{-1}$ somewhat larger than derived here. We are not aware of any published radial velocities for LS IV $+10^{\circ} 9$ and, therefore, conclude that there is insufficient observational material to judge whether LSS 1274 and LS IV $+10^{\circ} 9$ are radial velocity variable or not.

\section{Model atmospheres and synthetic spectra}

Visual spectra are well suited to derive the $T_{\text {eff }}, \log g$ and the helium abundance for example from the Pickering series of He II, $4686 \AA$, and several He I lines. Model atmospheres in NLTE and the corresponding synthetic spectra were calculated with the publicly available TLuSTY $200^{2}$ and Synspec $49^{3}$ codes (Hubeny \& Lanz 1995; Hubeny et al. 1994). The models are calculate in horizontally homogeneous, plane parallel geometry. A detailed description and manual is provided by Hubeny \& Lanz (2017a,b,c). The stellar atmospheres were assumed to be in radiative and hydrostatic equilibrium and include metal lineblanketing and the important broadening mechanisms. The most detailed model atoms were used that are available on the TLusty website. We used the default values of the standard keyword parameters.

We calculated three different model grids. A grid containing only hydrogen and helium was constructed in order to compare with previous results. In addition, two grids with $\mathrm{H} / \mathrm{He}$ and additional nitrogen or carbon (at the corresponding abundance for each sample star) were calculated to determine the atmospheric parameters and compare them to those derived by Hirsch (2009).

To fit the observational data, we made use of FITSB2 (Napiwotzki et al. 2004) spectral analysis program, and of SPAS, the Spectrum Plotting and Analysis Suite (Hirsch 2009), which are based on the fitting procedure described by Napiwotzki (1999). These programs allow us to measure and correct for the radial velocity shift of each spectrum and derive $T_{\text {eff }}$ and $\log g$ along with the abundance of a single element by comparing precalculated synthetic spectra to the observations by means of $\chi^{2}$-minimization. A spline fit is used to interpolate between the synthetic spectra, while the fitting itself and the error estimations are done via the downhill simplex algorithm. An overview about the model atoms used is given in Table 2. Including all significant elements $(\mathrm{H}, \mathrm{He}, \mathrm{C}, \mathrm{N}, \mathrm{O}, \mathrm{Ne}, \mathrm{Mg}$,

\footnotetext{
http: //nova . astro. umd. edu/Tlusty 2002

http://nova.astro.umd. edu/Synspec49/synspec.html
}

$\mathrm{Al}, \mathrm{Si}, \mathrm{P}, \mathrm{S}, \mathrm{Fe}, \mathrm{Ni}$ ) with their relevant model atoms in NLTE conditions is crucial to correctly model the structure of the stellar atmosphere and to improve the line fits in general.

Before starting the determination of atmospheric parameters and the abundance analysis, it is important to validate the fitting method and check if the calculated model atmospheres are appropriate. While determining abundances is quite straightforward, the atmospheric structure is often much harder to model.

\subsection{Model atmospheres and the effects of additional absorbers}

The determination of atmospheric parameters requires to properly account for line blanketing effects. As more metals are added, the total opacity increases and the effects of line blanketing back warming, and surface cooling are becoming more and more important, changing the temperature and density stratification of the atmosphere. Because accounting for many metal ions is demanding, very few attempts have been undertaken to model hot subdwarf atmospheres (e.g. by Lanz et al. 1997).

To investigate this issue, we compared the atmospheric structure delivered by different model grids. A model atmosphere with a temperature of $47000 \mathrm{~K}$, a surface gravity of $\log g=5.7^{4}$ and a helium enrichment of 100 times hydrogen by numbers (similar to the one of [CW83] 0904-02, for which C is the most abundant metal at ten times the solar abundance) was calculated. The abundances of all metals are listed in Table 8. The atmospheric structure was computed with an increasing number of opacity sources. The following steps were taken:

1. $\mathrm{H}, \mathrm{He}$

2. $\mathrm{H}, \mathrm{He}, \mathrm{C}$

3. $\mathrm{H}, \mathrm{He}, \mathrm{C}, \mathrm{N}, \mathrm{O}$

4. H, He, C, N, O, Ne, Mg, Al, Si, P, S

5. H, He, C, N, O, Ne, Mg, Al, Si, P, S, Fe, Ni

We use the full model (model 5) as our reference and plot the temperature distributions of the models $1(\mathrm{H} / \mathrm{He}$ only) to 5 (full model) after subtracting that of the reference model in Fig. 1. The stratification of the $\mathrm{H} / \mathrm{He}$ model (1) deviates drastically from that of the full model (5). As expected, adding absorbers cools down the outer atmospheric layers and heats up deeper layers significantly. However, in the line forming regions the temperature stratification of models 2-4 are almost identical and cooler than that of the full model (model 5) by $200 \mathrm{~K}-500 \mathrm{~K}$, only. We conclude that $\mathrm{H} / \mathrm{He} / \mathrm{C}$ composed model atmospheres with appropriately high abundances ( $\mathrm{He} / \mathrm{H}=100$ and ten times solar carbon) produce a realistic temperature stratification in the line forming region. The same holds for N-rich $\mathrm{He}$ sdO stars if we replace carbon by nitrogen in the model calculations for grid No. 2.

We considered an analysis of mock data more conclusive than the discussion of the temperature stratification. To this end we used the synthetic spectrum calculated from the full model (model 5) and fitted it with grids of synthetic spectra calculated from the less sophisticated models. The fit was performed using the $\mathrm{H} / \mathrm{He}$ and the $\mathrm{H} / \mathrm{He} / \mathrm{N}$ grid. The parameters were chosen to be the final ones for CD-31 $4800\left(T_{\text {eff }}=42230 \mathrm{~K}, \log g=5.6\right.$ and $\log y=\log (n(\mathrm{He}) / n(\mathrm{H})=2.6$, see Sect. 5). Accordingly, the nitrogen abundance was about ten times solar and the abundances of other metals are as given in Table 7.

Despite the large differences in the temperature stratification of the $\mathrm{H} / \mathrm{He}$ models, the best-fit effective temperature is hotter by $400 \mathrm{~K}$ than that of the full model, only (see Table 3). However,

\footnotetext{
4 Surface gravities are given in cgs units throughout this paper.
} 
Table 1. Radial velocities for the four sample stars with time of observation (start).

\begin{tabular}{|c|c|c|c|c|}
\hline Name & Date (UTC) & Instrument & Spectral range $(\AA)$ & $\mathrm{RV}\left(\mathrm{km} \mathrm{s}^{-1}\right)$ \\
\hline $\mathrm{CD}-31^{\circ} 4800$ & $2002-06-03 ; 23: 31.23$ & UVES & $3300 \ldots 6690$ & $30 \pm 2$ \\
\hline $\mathrm{CD}-31^{\circ} 4800$ & 2004-04-12;14:22:44 & FUSE & $905 \ldots 1185$ & $27 \pm 4 \pm 10$ \\
\hline [CW83] 0904-02 & 2005-02-24;04:24:19.13 & FEROS & $3530 \ldots 9200$ & $22 \pm 4$ \\
\hline [CW83] 0904-02 & $2005-02-24 ; 06: 20: 23.80$ & FEROS & $3530 \ldots 9200$ & $22 \pm 4$ \\
\hline [CW83] 0904-02 & $2004-05-04 ; 05: 19$ & FUSE & $905 \ldots 1185$ & $30 \pm 5 \pm 10$ \\
\hline [CW83] 0904-02 & 2004-05-05;04:34 & FUSE & $905 \ldots 1185$ & $30 \pm 5 \pm 10$ \\
\hline LSS 1274 & $2006-04-10 ; 00: 54$ & UVES & $3025 \ldots 6830$ & $13 \pm 1$ \\
\hline LSS 1274 & 2006-04-10;01:29 & UVES & $3025 \ldots 6830$ & $13 \pm 1$ \\
\hline LSS 1274 & $2002-03-11 ; 12: 13$ & FUSE & $905 \ldots 1185$ & $17 \pm 4 \pm 10$ \\
\hline LSS 1274 & 2002-03-08;06:00 & FUSE & $905 \ldots 1185$ & $16 \pm 4 \pm 10$ \\
\hline $\mathrm{LS} \mathrm{IV}+10^{\circ} 9$ & 2001-07-01;09:42:48 & UVES & $3280 \ldots 4560$ & $-32 \pm 3$ \\
\hline $\mathrm{LS}$ IV $+10^{\circ} 9$ & $2002-07-03 ; 17: 49$ & FUSE & $905 \ldots 1185$ & $-35 \pm 4 \pm 10$ \\
\hline $\mathrm{LS}$ IV $+10^{\circ} 9$ & 2004-05-10;18:11 & FUSE & $905 \ldots 1185$ & $-6 \pm 3 \pm 10$ \\
\hline
\end{tabular}

Notes. For radial velocities from FUSE data, we added the systematic uncertainty arising from wavelength calibration.

Table 2. Ionization stages for which detailed model atoms were used in the model atmosphere calculations.

\begin{tabular}{|c|c|c|c|c|c|}
\hline Element & $\mathrm{L}$ & SL & Element & $\mathrm{L}$ & SL \\
\hline $\mathrm{H}$ & 16 & 1 & $\mathrm{Mg}_{\text {II }}$ & 21 & 4 \\
\hline $\mathrm{He}_{\mathrm{I}}$ & 24 & 0 & $\mathrm{Al}$ III & 19 & 4 \\
\hline He II & 20 & 0 & Si III & 31 & 15 \\
\hline $\mathrm{C}_{\text {II }}$ & 34 & 5 & Si IV & 19 & 4 \\
\hline $\mathrm{C}_{\text {III }}$ & 34 & 12 & $\mathrm{P}_{\mathrm{IV}}$ & 14 & 0 \\
\hline C IV & 35 & 2 & $\mathrm{PV}_{\mathrm{V}}$ & 12 & 4 \\
\hline $\mathrm{N}_{\text {II }}$ & 32 & 10 & $S_{\text {III }}$ & 29 & 12 \\
\hline $\mathrm{N}_{\text {III }}$ & 39 & 9 & $\mathrm{~S}_{\text {IV }}$ & 29 & 12 \\
\hline N IV & 34 & 14 & S v & 20 & 5 \\
\hline $\mathrm{Nv}$ & 21 & 4 & S VI & 13 & 3 \\
\hline $\mathrm{O}_{\text {II }}$ & 36 & 12 & $\mathrm{Fe}$ III & 0 & 50 \\
\hline O III & 28 & 13 & Fe IV & 0 & 43 \\
\hline O IV & 31 & 8 & $\mathrm{Fe} v$ & 0 & 42 \\
\hline $\mathrm{Ne}$ II & 23 & 9 & Ni IV & 0 & 38 \\
\hline $\mathrm{Ne}$ III & 12 & 2 & $\mathrm{Niv}$ & 0 & 48 \\
\hline
\end{tabular}

Notes. The number of levels (L) and super-levels (SL) are listed. For each element the ground state of the next higher ionization stage was included, but not listed here.

the surface gravity is significantly larger and the helium abundance lower by $0.1 \mathrm{dex}$ and $0.8 \mathrm{dex}$, respectively.

$\mathrm{The} \mathrm{H} / \mathrm{He} / \mathrm{N}$ grid reproduces the spectrum calculated from the fully blanketed model very well. The atmospheric parameters of the best-fit $\mathrm{H} / \mathrm{He} / \mathrm{N}$ model differ by as little as $10 \mathrm{~K}, 0.02 \mathrm{dex}$ and 0.2 dex for $T_{\text {eff }}, \log g$ and $\log y$, respectively. This reassures us that the use of model grids including the most abundant metal $(\mathrm{C}$ or $\mathrm{N})$ at the appropriate high abundance is a good approximation to the computationally costly full models.

\section{Determination of effective temperature, surface gravity and abundances}

The effective temperature, surface gravity, and helium-tohydrogen ratio are determined from the hydrogen and helium lines listed in Table A.2. Another important atmospheric parameter is the abundance of the dominant metal (carbon and/or nitrogen, see Sect. 3). Other metals also have an impact on the

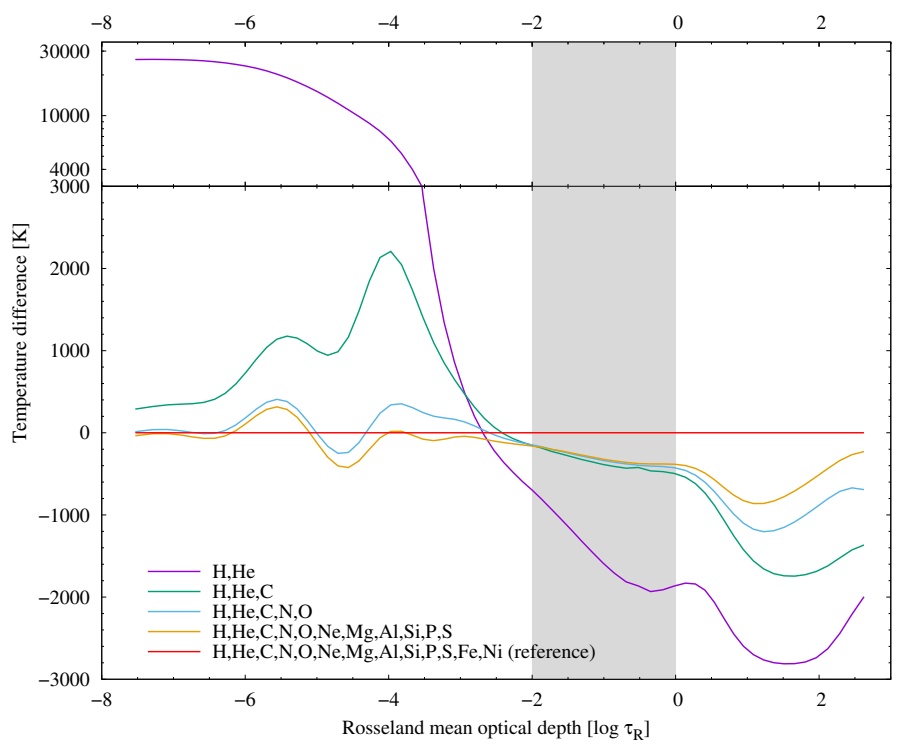

Fig. 1. Temperature stratification of TLusTy model atmospheres having different model atoms with respect to a fully line-blanketed reference model (No. 5). The shaded region marks the line forming region in the atmosphere. The y-axis shows the temperature difference with respect to the reference model, while the $\mathrm{x}$-axis gives the Rosseland mean optical depth $\tau_{\mathrm{R}}$. The figure demonstrates the effects of metal opacities in NLTE atmospheres. The higher the opacity (the more opacity sources included) the cooler the upper atmosphere and the hotter the deeper layers (surface cooling and back warming).

atmospheric temperature and density stratification, though at a lower level (see Sect. 3). Hence the following analysis strategy was designed.

We started with a pure $\mathrm{H} / \mathrm{He}$-grid in NLTE ranging from $35000 \mathrm{~K}$ to $56000 \mathrm{~K}$ in temperature and from 5.0 to 6.2 in $\log g$ to get a first estimate of the atmospheric parameters. The helium abundances covered the range from $n(\mathrm{He})=n(\mathrm{H})$ to $n(\mathrm{He})=$ $2000 \times n(\mathrm{H})$. The basic atmospheric parameters were determined with this grid. Because of the strong carbon and/or nitrogen line spectrum, many helium lines are blended with $\mathrm{C}$ and/or $\mathrm{N}$ lines (see Figs. 2 and A.1), those blends had to be accounted for. To that end a small sub-grid based on the preliminary atmospheric parameters was utilized to determine the carbon or 
Table 3. Spectral fit of a mock spectrum (parameters given in the 1 . row) by a grid of $\mathrm{H} / \mathrm{He}$ (2. row) and $\mathrm{H} / \mathrm{He} / \mathrm{N}$ (3. row) model spectra.

\begin{tabular}{llll}
\hline \hline Model & $T_{\text {eff }}(\mathrm{K})$ & $\log g$ & $\log y$ \\
\hline Mock data & 42230 & 5.60 & 2.60 \\
H/He fit & 42600 & 5.70 & 1.79 \\
H/He/N fit & 42290 & 5.58 & 2.40 \\
\hline
\end{tabular}

nitrogen abundance of each star, depending on which element showed stronger lines by visual inspections of the spectra. The next step was to upgrade the $\mathrm{H} / \mathrm{He}$ grid with carbon or nitrogen, using the previously determined value for the abundance. The $\mathrm{H} / \mathrm{He}$ grid was upgraded to $\mathrm{a} / \mathrm{He} / \mathrm{C}$ or a $\mathrm{H} / \mathrm{He} / \mathrm{N}$ grid (see Sect. 3.1). The additional element was included in NLTE conditions. With this new grid, the atmospheric parameters $\left(T_{\text {eff }}\right.$, $\log g, n(\mathrm{He}) / n(\mathrm{H}))$ were revised. This procedure improved the line fits considerably. In the next step we added additional metals by calculating new model atmospheres with solar abundance (Asplund et al. 2009) for each available additional "visual/UVA" element $(\mathrm{O}, \mathrm{Ne}, \mathrm{Mg}, \mathrm{Al}, \mathrm{Si}, \mathrm{P}, \mathrm{S})$ as a starting point for the further analysis and the atmospheric parameters from the previous iteration step were utilized. For each element a wide range of abundances was chosen in order to avoid extrapolation in the process of fitting individual lines. Afterwards, the abundances of all metals were calculated and fitted for one element after the other to the respective selected visual, UVA, and FUV lines.

The procedure involved a simultaneous fit to the lines of the particular ion and uncertainties were determined using the bootstrapping method (see Napiwotzki 1999). After having determined the final abundance of one element, the next element was investigated. This means that all elements included in the model have an influence on the stratification of the atmosphere. Elements with a high expected abundance (i.e. from visual inspection of the spectra) were fitted first. These were the elements of $\mathrm{C}$, $\mathrm{N}, \mathrm{O}$ and $\mathrm{Ne}$ or $\mathrm{Si}$, while elements with hardly any visible lines like $\mathrm{S}$ or $\mathrm{P}$ were fitted last. To check for consistency between the visual, UVA and the FUV data and to improve the results, the fits were recalculated once more. This time iron and nickel were included from the beginning in the line formation and both the visual/UVA and FUV data were fitted for each ion. The final abundance is the error-weighted average of the ion-based abundances from the visual/UVA and the FUV. In those cases, where elements were only detectable in the visual/UVA, but not in the FUV, or vice versa, the abundance results are derived from only one wavelength range.

\section{Effective temperatures, surface gravity, and Helium-to-Hydrogen ratio}

The first step was to determine the atmospheric parameters of each star from the visual helium/hydrogen lines (see Table A.2). The first parameter estimate was done with a pure $\mathrm{H} / \mathrm{He}$ grid. In the second step a $\mathrm{H} / \mathrm{He}+\mathrm{C}$ or $\mathrm{H} / \mathrm{He}+\mathrm{N}$ grid was used which were tailored to the specific object. Figure 2 shows the fit to the hydrogen and helium lines for CD-3 $1^{\circ} 4800$ and LSS 1274 . The fits for [CW83] 0904-02 and LS IV $+10^{\circ} 9$ are shown in Figs. A.1 and A.2. If possible, the same set of spectral lines was used in all stars. However, this was not always possible because of wavelength gaps, or artifacts in the spectra. Because of the low hydrogen abundances the Balmer lines introduce small asymmetries to every second line in the He II Pickering series. At the appropriate He-to- $\mathrm{H}$ ratios all models predict $\mathrm{H} \alpha$ emission. For the nitrogen-rich $\mathrm{CD}-31^{\circ} 4800$ this component can be reproduced very well (see Fig. 2). Other Balmer lines have to be reproduced simultaneously and consistently as well. In this context it is worthwhile to note that the core of the $\mathrm{He} \mathrm{I} / \mathrm{H} \beta$ line is blended by a nitrogen triplet, which is non-negligible contribution to the line profile and has to be accounted for at high nitrogen abundances. However, the C-rich targets LSS 1274 and [CW83] 0904-02 do not show $\mathrm{H} \alpha$ emission ${ }^{5}$ components (see Figs. 2 and A.1). Hence, we are unable to reproduce this feature. Reducing the hydrogen content would remedy the issue, but the other lines of the Balmer series could not be reproduced. Hence, we rely on the higher Balmer lines.

Stroeer et al. (2007) analyzed the high-resolution spectra of the SPY-sdO sample and found that $1-\sigma$ statistical errors are typically lower than $100 \mathrm{~K}, 0.04$, and 0.04 for $T_{\text {eff }}, \log g$, and $\log y$, respectively. However, the error budget is dominated by systematic uncertainties. In the SPY project each target star was observed at least twice. Stroeer et al. (2007) estimated average systematic uncertainties by evaluating the distribution of differences in the fit parameters from individual spectra and find $1 \sigma$-values (sample standard deviation) of these distributions of $\Delta T_{\text {eff }} / T_{\text {eff }}=0.026, \Delta \log g=0.097$ and $\Delta \log y / \log y=0.097$ for the sample. For our stars test calculations demonstrate that effective temperatures are better constrained by several ionization equilibria and the excellent quality of our spectra. However, the gravity uncertainties derived by Stroeer et al. (2007) appear to be realistic, while that of the $\mathrm{He} / \mathrm{H}$ ratio appears to be underestimated for the extremely helium-rich stars. The finally adopted uncertainties are given in Table 4 which summarizes the results for the effective temperature, surface gravity, helium-tohydrogen number ratio $(\log (y))$, as well as the number ratio of the most abundant metal $((\log (X / H))$, that is $\mathrm{N}$ in the case of $\mathrm{CD}-31^{\circ} 4800$, and $\mathrm{C}$ for the others) with respect to hydrogen. The rotation velocity as derived from metal lines (see Sect. 7.3) is also given. Because LS IV $+10^{\circ} 9$ is carbon and nitrogen-rich, we fitted the star with the N-rich model grid as well with the $\mathrm{C}$-rich grid. The resulting atmospheric parameters are very similar (see Table 4).

[CW83] 0904-02 has been analyzed by Hirsch (2009) from the same FEROS visual spectra used here with synthetic spectra calculated with the TMAP code and hydrogen, helium and C composition, respectively. Hirsch (2009) also analyzed CD$31^{\circ} 4800$ from a FEROS spectrum. Hence, it is worthwhile to compare his results to ours (see Table 4).

For [CW83] 0904-02 our analysis yields an effective temperature higher than that derived by Hirsch (2009) by about $800 \mathrm{~K}$. For CD-31 4800 both the effective temperature and the surface gravity are lower than derived by Hirsch (2009).

Figure 3 compares the position of our program stars to the He-sdOs analyzed by Hirsch (2009) in the $T_{\text {eff }}, \log g$ diagram. All stars lie slightly above the helium main sequence. The $\mathrm{N}$-rich stars including CD-31 4800 are cooler than the C-rich ones including LSS 1274, [CW83] 0904-02, and LS IV +10 9 , while both subclasses of He-sdOs have similar gravities.

\section{Angular diameters, interstellar reddening, and stellar masses}

Once the atmospheric parameters have been determined, photometric measurements allow additional parameters to be

The $\mathrm{H} \alpha$ line is not observed for $\mathrm{LS}$ IV $+10^{\circ} 9$. 
M. Schindewolf et al.: A quantitative NLTE analysis of four helium-rich subdwarf O stars
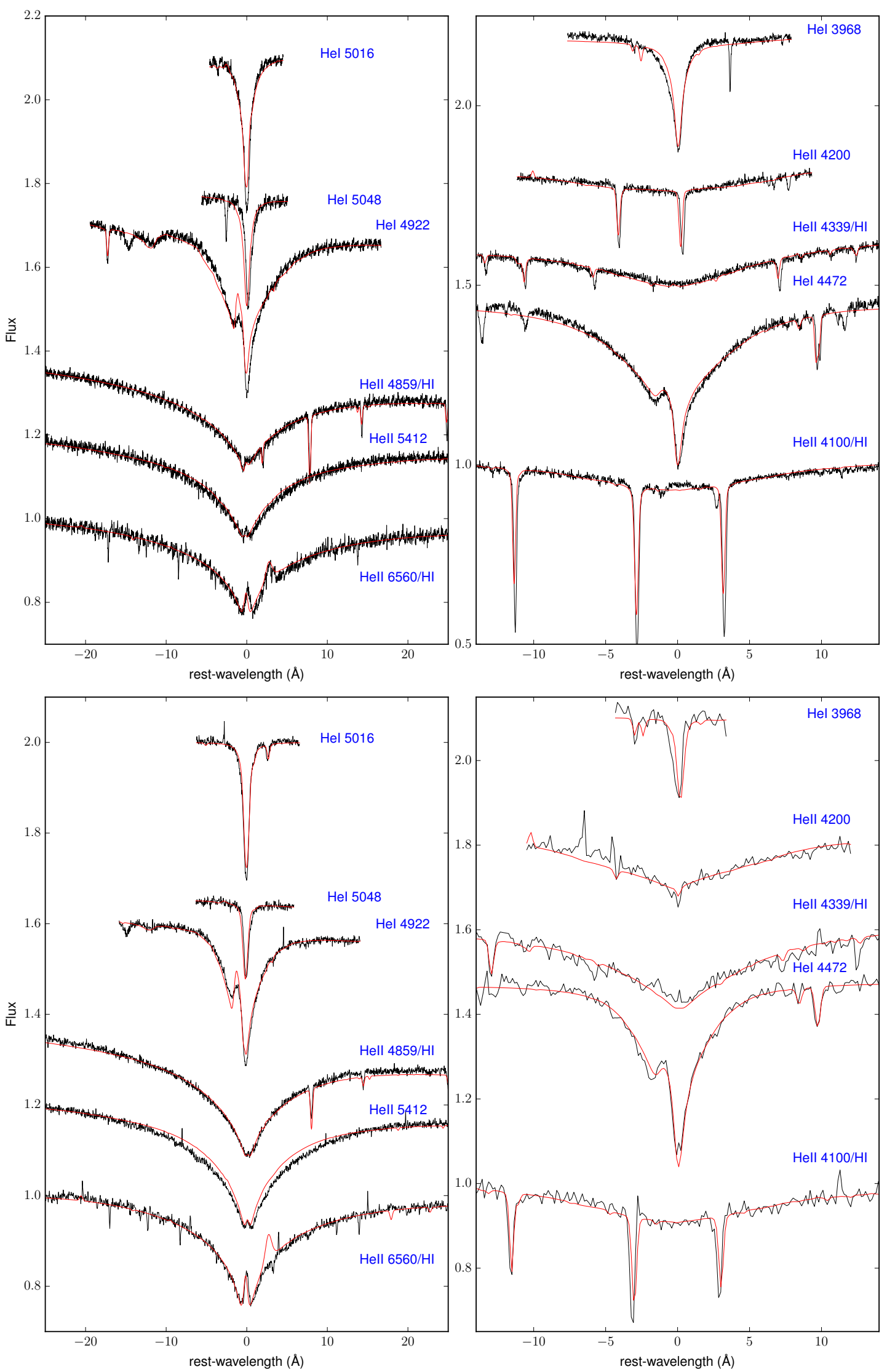

Fig. 2. He I and He II line profiles from the final models compared to the normalized observed spectra of CD-31 4800 (UVES, top panels) and LSS 1274 (bottom panels, UVES left, CASPEC right). The spectra have been corrected for radial velocity. Because it was not possible to match the $\mathrm{He}$ II/ $/ \mathrm{H}_{\mathrm{I}} 6560$ line in LSS 1274 it was not included in the fit and is only shown for comparison. The very sharp absorption lines in the 6560 line wings are telluric lines. 
Table 4. Effective temperatures, surface gravities, $\mathrm{H} / \mathrm{He}$ number ratio, abundance of the dominant metal $\mathrm{X}$ and projected rotational velocity $v_{\text {rot }} \sin i$ of the sample stars, compared with the results of Hirsch (2009).

\begin{tabular}{clcccccc}
\hline \hline Name & $\begin{array}{l}T_{\text {eff }} \\
(\mathrm{K})\end{array}$ & $\begin{array}{c}\log g \\
(\mathrm{cgs})\end{array}$ & $\log y$ & $\log (X / H)$ & $\begin{array}{c}v_{\text {rot }} \sin i \\
\left(\mathrm{~km} \mathrm{~s}^{-1}\right)\end{array}$ & Spectrum & Ref. \\
\hline CD-31 4800 & $42230 \pm 300$ & $5.60 \pm 0.1$ & $2.61 \pm 0.20$ & $-0.31 \pm 0.11(\mathrm{~N})$ & $<5$ & UVES & 1 \\
& $43000 \pm 250$ & $5.84 \pm 0.05$ & $2.75 \pm 0.30$ & $-0.29 \pm 0.17(\mathrm{~N})$ & $<10$ & FEROS & 2 \\
LSS 1274 & $44270 \pm 400$ & $5.48 \pm 0.10$ & $2.17 \pm 0.25$ & $-0.01 \pm 0.19(\mathrm{C})$ & $<5$ & UVES & 1 \\
[CW83] 0904-02 & $47000 \pm 500$ & $5.70 \pm 0.10$ & $2.0 \pm 0.3$ & $-0.11 \pm 0.13(\mathrm{C})$ & $32 \pm 3$ & UVES & 1 \\
& $46170 \pm 250$ & $5.64 \pm 0.05$ & $1.91 \pm 0.3$ & $-0.12 \pm 0.2(\mathrm{C})$ & $30 \pm 4$ & FEROS & 2 \\
LS IV +10 9 & $43850 \pm 200$ & $5.43 \pm 0.1$ & $2.73 \pm 0.25$ & $+0.15 \pm 0.13(\mathrm{C})$ & $<5$ & UVES & 1 \\
& $44000 \pm 250$ & $5.42 \pm 0.1$ & $2.76 \pm 0.20$ & $-0.13 \pm 0.27(\mathrm{~N})$ & $<5$ & UVES & 1 \\
\hline
\end{tabular}

References. $1=$ this work, $2=$ Hirsch (2009).

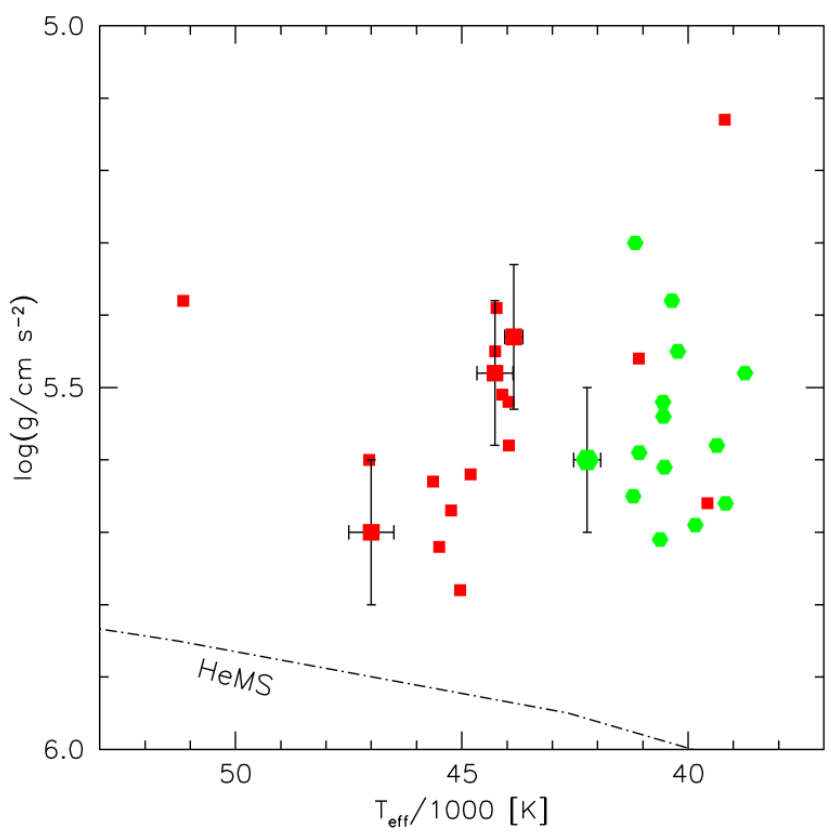

Fig. 3. Comparison of the position of the program stars in a $T_{\text {eff }}, \log g$ diagram to that the sample of Hirsch (2009). Stars with nitrogen dominated atmospheres are shown as green hexagons, while red square denote stars with carbon dominated atmospheres. The helium main sequence (Paczyński 1971) is shown for reference.

determined, in particular the stellar angular diameter and the interstellar extinction. If the distance is known, the stellar mass can be derived. To this end we constructed spectral energy distributions from photometric measurements ranging from the ultraviolet (IUE) to the infrared $(J, H, K ; 2$ MASS ,UKIDS, Skrutskie et al. 2006; Lawrence et al. 2007) and (W1,W2; WISE, Cutri 2013). Magnitudes and colors in the Johnson (Mermilliod et al. 1997; Landolt 2007), Strömgren (Paunzen 2015), Tycho (Høg et al. 2000), and SDSS (Henden et al. 2016; Wolf et al. 2018) photometric systems were fitted along with the SEDs (for details see Heber et al. 2018). The photometric filter systems are inhomogeneous with respect to their bandwidth. Also the precision of the measured magnitudes may differ for the different filters. Ultraviolet spectra from IUE cover the wavelength range from $1150 \AA$ to $3150 \AA$ at a spectral resolution of $6 \AA$, whereas visual and infrared magnitudes are derived both from narrow band (e.g. Strömgren) and wide-band (e.g. Johnson, 2MASS, WISE) photometry.
Because we have to combine UV spectrophotometry from IUE with broad and intermediate band visual and infrared photometry a suitable set of filters were defined to derive UVmagnitudes from IUE spectra that comprises of three box filters, which cover the spectral ranges 1300-1800 $\AA$, 2000$2500 \AA$, and $2500-3000 \AA$. Interstellar extinction is accounted for by multiplying the synthetic flux with a reddening factor $10^{-0.4 \mathrm{~A}(\lambda)}$ using the extinction curve of Fitzpatrick (1999). We match the synthetic SED calculated from the final model to the observed one by adjusting the angular diameter and the interstellar reddening parameter $E(B-V)$ assuming an extinction parameter $R_{\mathrm{V}}=A(V) / E(B-V)=3.15$. In Figs. 4 (LSS 1274) and A.4 (CD-3 $1^{\circ} 4800$, LS IV $+10^{\circ} 9$, and [CW83] 0904-02) we display the residuals (O-C) of the SED and color fits and plot the SEDs as flux density times the wavelength to the power of three $\left(F_{\lambda} \lambda^{3}\right)$ as a function of wavelength to reduce the steep slope of the SED on such a broad wavelength range.

The resulting angular diameter and interstellar reddening parameters are listed in Table 5. Because of the quite low Galactic distances $z$ of the program stars from the Galactic plane, we expect interstellar reddening to be substantial. Therefore, we also give the reddening to infinity from Schlafly \& Finkbeiner (2011b). The reddening for [CW83] 0904-02 is consistent while for the three others it is found to be significantly less than derived from the maps of Schlafly \& Finkbeiner (2011b) indicating that most of the reddening takes place beyond the stars' distances. Reddening parameters for [CW83] 0904-02 and LS IV +10 9 can be estimated from the $3 \mathrm{D}$ dust maps of Green et al. (2018) and are consistent with those derived from the SED. Figures 4 (LSS 1274) and A.4 (CD-31 4800 , LS IV $+10^{\circ} 9$, and [CW83] 0904-02) demonstrate that the synthetic SEDs reproduce the observed ones all the way from the ultraviolet to the infrared. No infrared excess becomes apparent for any of our program stars. For LS IV $+10^{\circ} 9$ this is at odds with the results by Ulla \& Thejll (1998) who found an infrared excess for LS IV $+10^{\circ} 9$ and concluded that this star has a companion of spectral type F4 to F6. However, their infrared photometry are brighter by up to two magnitudes than the 2MASS photometry we used (see Table 6). We have no reason to believe that the star is variable in light and conclude that the claimed IR excess is spurious.

The recent, second data release of Gaia (Gaia Collaboration 2018) provided high-precision parallaxes $\varpi$ for the program stars. Hence we can derive the stellar mass from the parallax, the angular diameter and the surface gravity via

$M=g \Theta^{2} /\left(4 G \varpi^{2}\right)$. 


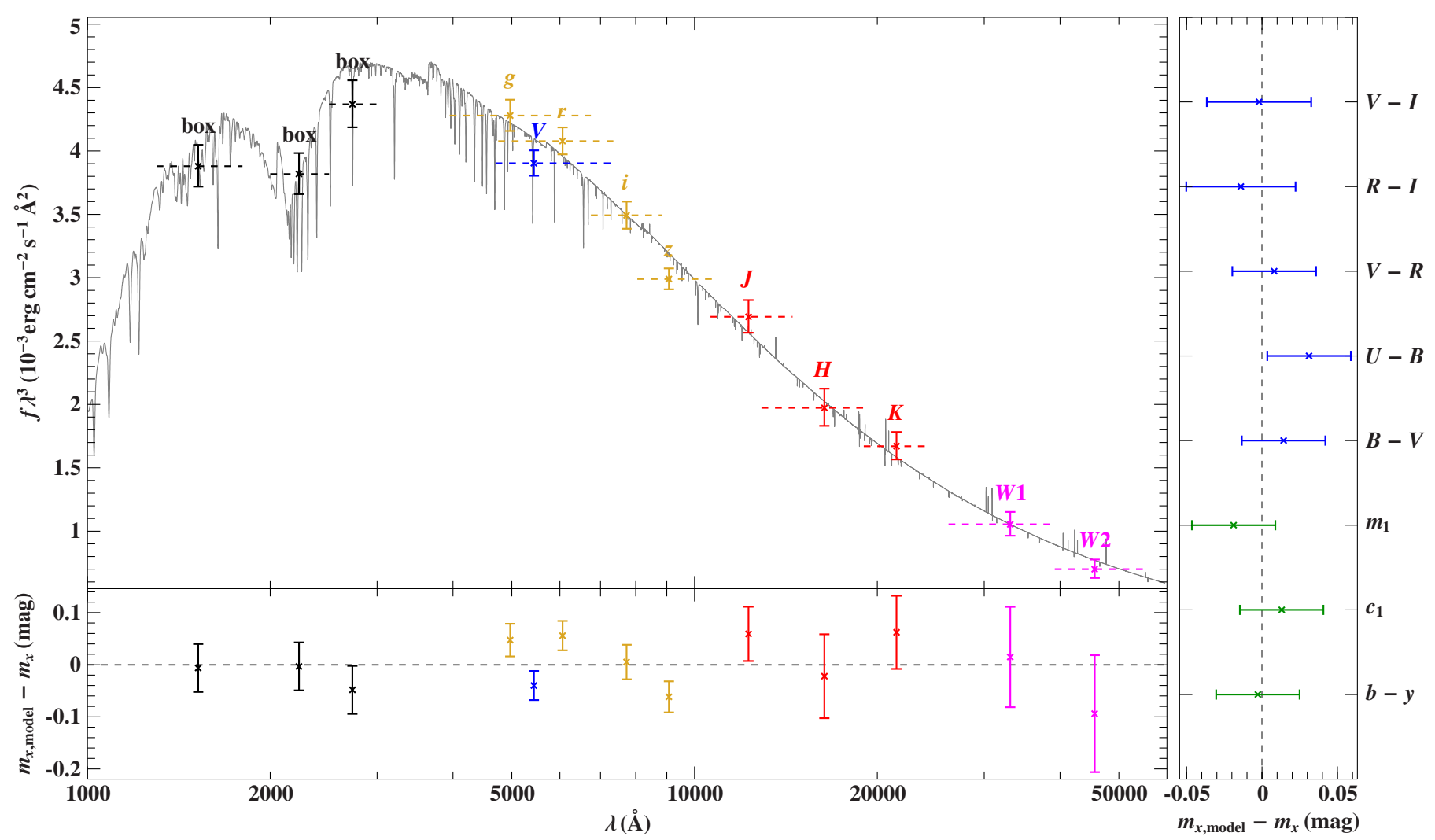

Fig. 4. Comparison of synthetic and observed photometry for LSS 1274: The top panel shows the spectral energy distribution. The three black data points labeled "box" are fluxes converted from artificial magnitudes computed by means of box filters of the indicated width from a lowdispersion IUE spectrum. Filter-averaged fluxes are shown as colored data points which were converted from observed magnitudes (the dashed horizontal lines indicate the respective filter widths), while the gray solid line represents a synthetic spectrum computed from a model atmosphere using the atmospheric parameters given in Table 4. The residual panels at the bottom and right hand side show the differences between synthetic and observed magnitudes/colors. The following color codes are used to identify the photometric systems: Johnson-Cousins (blue), Strömgren (green), SkyMapper (yellow), 2MASS (red), WISE (magenta).

Table 5. Angular diameter $\Theta$, interstellar reddening $A_{\mathrm{V}}$, interstellar reddening to infinity $A_{\mathrm{V}_{\mathrm{S}}}($ Schlafly \& Finkbeiner 2011a).

\begin{tabular}{llllll}
\hline \hline Star & $\Theta$ & $A_{\mathrm{V}}$ & $A_{\mathrm{V}_{\mathrm{S}}}$ & Parallax $\varpi(\mathrm{mas})$ & Mass $\left(M_{\odot}\right)$ \\
\hline CD-31 ${ }^{\circ} 4800$ & $3.561 \times 10^{-11}$ & 0.056 & 1.678 & $4.8619 \pm 0.0642$ & $0.38 \pm 0.09$ \\
LSS 1274 & $1.298 \times 10^{-11}$ & 0.339 & 1.683 & $1.4618 \pm 0.0461$ & $0.43 \pm 0.10$ \\
LS IV +10 9 & $1.809 \times 10^{-11}$ & 0.164 & 0.196 & $2.041 \pm 0.0628$ & $0.38 \pm 0.09$ \\
[CW83] 0904-02 & $1.742 \times 10^{-11}$ & 0.066 & 0.063 & $2.1574 \pm 0.0737$ & $0.59 \pm 0.14$ \\
\hline
\end{tabular}

Notes. The Gaia parallaxes $\varpi$ and the stellar masses are given in the last two columns.

Table 6. Comparison of photometric magnitudes $J, H$ and $K$ from Ulla \& Thejll (1998) and Cutri et al. (2003).

\begin{tabular}{ccc}
\hline & Ulla \& Thejll (1998) & Cutri et al. (2003) \\
\hline$J$ & $11.15 \pm 0.03$ & $12.632 \pm 0.03$ \\
$H$ & $10.98 \pm 0.05$ & $12.813 \pm 0.02$ \\
$K$ & $10.90 \pm 0.05$ & $12.916 \pm 0.04$ \\
\hline
\end{tabular}

The uncertainty of the mass is

$\Delta M / M=\mathrm{d} \ln M=\sqrt{(A \Delta \log g)^{2}+4\left(\frac{\Delta \Theta}{\Theta}\right)^{2}+4\left(\frac{\Delta \varpi}{\varpi}\right)^{2}}$,

$(A=\ln 10)$, which for the program stars is dominated by the uncertainty of the gravity. The masses, listed in Table 5, scatter around the canonical mass for the core helium flash with CD$31^{\circ} 4800$ and $\mathrm{LS} \mathrm{IV}+10^{\circ} 9$ at the low end and [CW83] 0904-02 at the high one.

\section{Metal abundances}

Having determined the atmospheric parameters we proceed to derive metal abundances. We used the NLTE synthetic spectra calculated with TLuSTY/SynsPec to derive the elemental abundance of $\mathrm{C}, \mathrm{N}, \mathrm{O}, \mathrm{Ne}, \mathrm{Mg}, \mathrm{Si}, \mathrm{P}, \mathrm{S}, \mathrm{Fe}$, and $\mathrm{Ni}$ from selected lines and line blends were added subsequently in the spectral synthesis (see Sect. 2.2). Finally, the synthetic spectra were compared to the observed spectra to identify outliers. Many ions display spectral lines in the visual/UVA as well as in the FUSE spectral range. This allows a comparison of abundances derived from different spectral ranges to be made, 


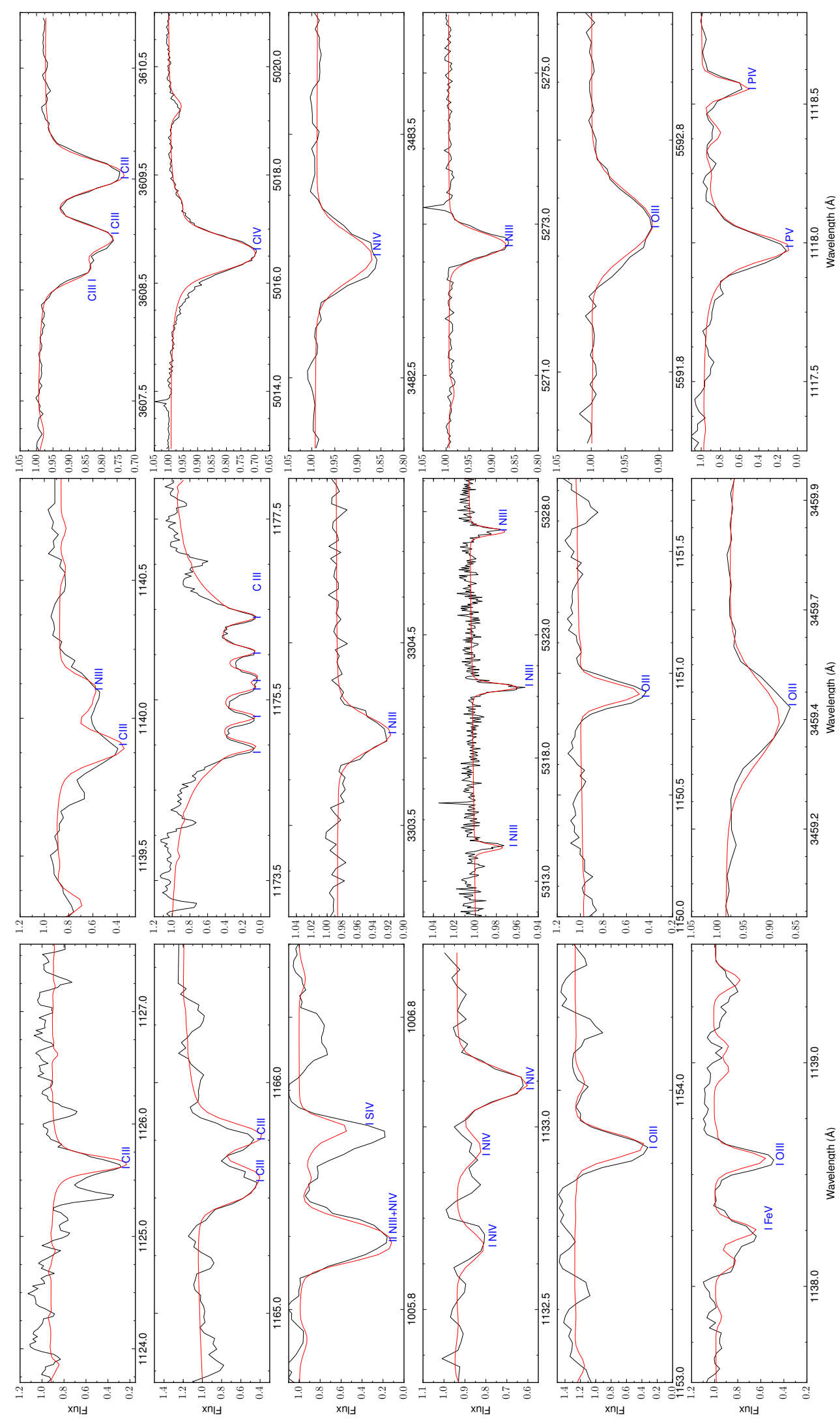

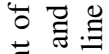

范志

㝵范

क्ष

(3)

翌

岂

$\dot{\mathrm{s}} \stackrel{\mathrm{E}}{\mathrm{g}}$

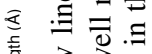

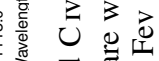

完

园

$\ddot{3}$ 苛

气

$\therefore$, 00

究的

$58:$

究

की

के

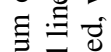

鸪

क्ष

这定

害不

뗀 3

空

吾吉

인

政

สํํㅇ 要焉

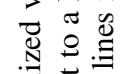

可 글 담 o 면 응 츨

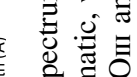

空

政

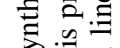

5.

范

0 응

t大०<

ป

总

สิ घี

نे

in

证导艺造 
Table 7. Surface abundances of CD-31 4800 (left hand panel) and for LSS 1274 (right hand panel) as derived from visual/UVA and FUV data. The values are given as $\log (n(\mathrm{X}) / n(\mathrm{H}))$.

\begin{tabular}{|c|c|c|c|c|c|c|c|}
\hline \multicolumn{4}{|c|}{$\mathrm{CD}-31^{\circ} 4800$} & \multicolumn{4}{|c|}{ LSS 1274} \\
\hline Element & Visual/UVA & FUSE & Adopted & Element & Visual/UVA & FUSE & Adopted \\
\hline He I \& He II & $2.61 \pm 0.20$ & - & $2.61 \pm 0.20$ & He I \& He II & $2.17 \pm 0.25$ & - & $2.17 \pm 0.25$ \\
\hline $\mathrm{C}_{\text {III }}$ & $-2.30 \pm 0.11$ & $-1.72 \pm 0.15$ & & $\mathrm{C}_{\text {III }}$ & $-0.01 \pm 0.06$ & $0.11 \pm 0.11$ & \\
\hline $\mathrm{C}_{\mathrm{IV}}$ & $-2.09 \pm 0.04$ & $-1.84 \pm 0.21$ & & $\mathrm{C}_{\mathrm{IV}}$ & $-0.14 \pm 0.08$ & $0.07 \pm 0.34$ & \\
\hline $\mathbf{C}$ & $-2.14 \pm 0.13$ & $-1.76 \pm 0.18$ & $-2.01 \pm 0.16$ & $\mathbf{C}$ & $-0.06 \pm 0.12$ & $0.09 \pm 0.25$ & $-0.01 \pm 0.19$ \\
\hline $\mathrm{N}_{\text {II }}$ & $-0.25 \pm 0.06$ & - & & $\mathrm{N}_{\text {II }}$ & $(-1.29 \pm 0.71)$ & - & \\
\hline $\mathrm{N}_{\text {III }}$ & $-0.24 \pm 0.02$ & $-0.37 \pm 0.12$ & & $\mathrm{~N}_{\text {III }}$ & $-1.19 \pm 0.06$ & $-1.07 \pm 0.36$ & \\
\hline Niv & $-0.27 \pm 0.02$ & $-0.41 \pm 0.12$ & & $\mathrm{~N}_{\text {IV }}$ & $-1.01 \pm 0.21$ & $-1.05 \pm 0.11$ & \\
\hline $\mathbf{N}$ & $-0.25 \pm 0.10$ & $-0.39 \pm 0.12$ & $-0.31 \pm 0.11$ & $\mathbf{N}$ & $-1.15 \pm 0.17$ & $-1.06 \pm 0.26$ & $-1.11 \pm 0.22$ \\
\hline $\mathrm{O}_{\text {II }}$ & $-2.09 \pm 0.04$ & - & & $\mathrm{O}_{\text {II }}$ & $(-1.60 \pm 0.80)$ & - & \\
\hline $\mathrm{O}_{\text {III }}$ & $-2.29 \pm 0.05$ & $-2.12 \pm 0.08$ & & $\mathrm{O}_{\text {III }}$ & $-1.90 \pm 0.22$ & $-1.75 \pm 0.21$ & \\
\hline O IV & - & $-2.58 \pm 0.28$ & & O IV & - & $(-1.40 \pm 0.59)$ & \\
\hline $\mathbf{O}$ & $-2.19 \pm 0.11$ & $-2.24 \pm 0.14$ & $-2.21 \pm 0.13$ & $\mathbf{O}$ & $-1.90 \pm 0.24$ & $-1.75 \pm 0.23$ & $-1.82 \pm 0.24$ \\
\hline $\mathrm{Ne}$ II & $-0.98 \pm 0.10$ & $-0.62 \pm 0.14$ & $-0.83 \pm 0.12$ & $\mathrm{Ne}$ II & $-0.70 \pm 0.11$ & $-0.76 \pm 0.31$ & $-0.71 \pm 0.21$ \\
\hline $\mathrm{Mg}_{\text {II }}$ & $-1.56 \pm 0.10$ & - & $-1.56 \pm 0.10$ & $\mathrm{Mg}_{\text {II }}$ & $-1.96 \pm 0.29$ & - & $-1.96 \pm 0.29$ \\
\hline Al III & $-2.61 \pm 0.10$ & $-2.93 \pm 0.32$ & $-2.68 \pm 0.21$ & Al III & $-1.93 \pm 0.31$ & $-2.25 \pm 0.30$ & $-2.09 \pm 0.30$ \\
\hline $\mathrm{Si}$ III & $-1.57 \pm 0.10$ & $-1.75 \pm 0.14$ & & $\mathrm{Si}$ III & $-2.23 \pm 0.21$ & $-2.19 \pm 0.25$ & \\
\hline Si IV & $-1.78 \pm 0.15$ & $-1.75 \pm 0.18$ & & Si IV & $-2.23 \pm 0.14$ & $-2.08 \pm 0.03$ & \\
\hline $\mathbf{S i}$ & $-1.65 \pm 0.16$ & $-1.75 \pm 0.16$ & $-1.70 \pm 0.16$ & $\mathbf{S i}$ & $-2.23 \pm 0.20$ & $-2.09 \pm 0.17$ & $-2.16 \pm 0.19$ \\
\hline$P_{\text {IV }}$ & - & $-3.92 \pm 0.11$ & & $\mathrm{P}_{\mathrm{IV}}$ & - & $-4.02 \pm 0.19$ & \\
\hline $\mathrm{Pv}_{\mathrm{v}}$ & - & $-3.83 \pm 0.13$ & & $\mathrm{Pv}_{\mathrm{v}}$ & - & $-3.80 \pm 0.11$ & \\
\hline $\mathbf{P}$ & - & $-3.88 \pm 0.15$ & $-3.88 \pm 0.15$ & $\mathbf{P}$ & - & $-3.80 \pm 0.15$ & $-3.80 \pm 0.15$ \\
\hline$S_{\text {III }}$ & $-1.79 \pm 0.03$ & - & & S III & - & - & \\
\hline S IV & $-2.22 \pm 0.07$ & $-2.09 \pm 0.10$ & & S IV & $-2.46 \pm 0.06$ & $-2.54 \pm 0.13$ & \\
\hline $\mathrm{S} v$ & - & $-1.87 \pm 0.41$ & & $\mathrm{~S} v$ & - & $-2.32 \pm 0.39$ & \\
\hline $\mathrm{S}_{\mathrm{V}}$ & - & - & & S VI & - & $(-2.41 \pm 0.70)$ & \\
\hline $\mathbf{S}$ & $-1.93 \pm 0.11$ & $-2.05 \pm 0.27$ & $-1.96 \pm 0.19$ & $\mathbf{S}$ & $-2.46 \pm 0.11$ & $-2.49 \pm 0.28$ & $-2.47 \pm 0.19$ \\
\hline $\mathbf{F e}$ & - & $-2.01 \pm 0.27$ & $-2.01 \pm 0.27$ & $\mathbf{F e}$ & - & $-2.42 \pm 0.18$ & $-2.42 \pm 0.18$ \\
\hline $\mathbf{N i}$ & - & $-2.49 \pm 0.23$ & $-2.49 \pm 0.23$ & $\mathbf{N i}$ & - & $-2.62 \pm 0.29$ & $-2.62 \pm 0.29$ \\
\hline
\end{tabular}

Notes. A "-" indicates that no usable lines of the element were found. Abundance are listed as derived from each ion accessible. A bold element symbol indicates the abundance from all ionization stages combined. The finally adopted abundances are the error-weighted averages of the results from the visual/UVA and FUSE spectra, if available. Abundances with uncertainties exceeding 0.5 dex are given in parentheses (see text).

which is a crucial cross-check for the reliability of FUV results which suffer from line crowding and uncertainties of continuum adjustment. For iron and nickel we have to rely on the analyses of FUV spectra. In Fig. 5 we compare the final synthetic spectrum to the observed spectra of LSS 1274 by selecting lines of different strengths, ionization stages and from different wavelength regimes to demonstrate the quality of fits achieved.

Several chemical elements (C, N, O, Si, P, S, Fe, and Ni) display spectral lines arising from two or three stages of ionization. Because ionization equilibria are very sensitive to effective temperature, the inspection of the chemical abundances derived from different ions is an important check on the effective temperatures derived from the $\mathrm{He}_{\mathrm{I}}$ and $\mathrm{He}$ II lines. As demonstrated in Tables 7 and 8 the ionization equilibria for almost all elements are intact for the four stars, irrespective of whether they were obtained from the visual or the FUSE spectra. Elemental abundances are given as the error averaged mean values derived from different stages of ionization if available. The comparison of abundances derived from the visual/UVA to that from the FUV is also presented in Tables 7 and 8. There seems to be a slight tendency for abundances from the FUV to be larger than those from the visual/UVA by about $0.2 \mathrm{dex}$ in the case of [CW83] 0904-02. However, for the other three stars the difference between FUSE and visual abundance scatter around zero by about 0.2 dex in most cases. Hence, we conclude that abundances derived from FUSE and and visual/UVA spectra are consistent to within error margins. The finally adopted values are the error weighted averages from both spectral ranges, the errors are the average from the single errors. The finally adopted abundances were derived from error weighted averages of abundances from the different wavelength regimes if available. Test calculation have shown that the uncertainties of the atmospheric parameters have a minor effect on the elemental abundances, except for $\mathrm{O}, \mathrm{Ne}$ and $\mathrm{Ni}$ lines in the FUV. A very conservative estimate of the corresponding error is \pm 0.1 dex for the results from the visual/UVA. The same number is appropriate for the FUV range for all elements but $\mathrm{O}, \mathrm{Ne}$, and $\mathrm{Ni}$, for which we adopt 0.15 dex. The adopted abundance error is calculated by adding these numbers in quadrature before averaging the abundances from the two wavelength ranges. Abundances with uncertainties exceeding 0.5 dex are not included in the averaging procedure.

\subsection{Abundance by element}

The available spectra and NLTE model atoms for TLUSTY/SYNSPEC enabled us to derive elemental abundance 
Table 8. Same as Table 7, but for [CW83] 0904-02 (left hand panel) and LS IV $+10^{\circ} 9$ (right hand panel).

\begin{tabular}{|c|c|c|c|}
\hline \multicolumn{4}{|c|}{ [CW83] 0904-02 } \\
\hline Element & Visual/UVA & FUSE & Adopted \\
\hline $\mathrm{He}_{\mathrm{I}} \& \mathrm{He}_{\mathrm{II}}$ & $2.0 \pm 0.3$ & - & $2.0 \pm 0.3$ \\
\hline $\mathrm{C}_{\mathrm{III}}$ & $-0.44 \pm 0.07$ & $0.09 \pm 0.06$ & \\
\hline CiV & $0.11 \pm 0.05$ & $-0.13 \pm 0.27$ & \\
\hline $\mathbf{C}$ & $-0.17 \pm 0.06$ & $0.05 \pm 0.19$ & $-0.11 \pm 0.13$ \\
\hline $\mathrm{N}_{\text {II }}$ & - & - & \\
\hline $\mathrm{N}_{\text {III }}$ & $-1.69 \pm 0.04$ & $-1.49 \pm 0.08$ & \\
\hline $\mathrm{N}_{\text {IV }}$ & $-1.99 \pm 0.37$ & $-1.53 \pm 0.11$ & \\
\hline $\mathbf{N}$ & $-1.70 \pm 0.23$ & $-1.51 \pm 0.14$ & $-1.58 \pm 0.19$ \\
\hline $\mathrm{O}_{\text {II }}$ & $-2.07 \pm 0.37$ & & \\
\hline $\mathrm{O}_{\text {III }}$ & $-2.34 \pm 0.16$ & $-1.94 \pm 0.13$ & \\
\hline O IV & - & $(-2.18 \pm 0.57)$ & \\
\hline $\mathbf{O}$ & $-2.25 \pm 0.28$ & $-1.94 \pm 0.16$ & $-2.05 \pm 0.22$ \\
\hline $\mathrm{Ne}_{\text {II }}$ & $-1.03 \pm 0.20$ & $-0.96 \pm 0.08$ & \\
\hline $\mathrm{Ne}$ III & - & $-0.94 \pm 0.05$ & \\
\hline $\mathrm{Ne}$ & $-1.03 \pm 0.22$ & $-0.95 \pm 0.12$ & $-0.97 \pm 0.16$ \\
\hline $\mathrm{Mg}_{\text {II }}$ & $-2.41 \pm 0.16$ & - & $-2.41 \pm 0.16$ \\
\hline $\mathrm{Al}$ III & $-3.05 \pm 0.23$ & $(-2.91 \pm 0.62)$ & $-3.05 \pm 0.23$ \\
\hline Si III & - & $-2.43 \pm 0.30$ & \\
\hline Si IV & $-2.58 \pm 0.08$ & $(-3.15 \pm 0.54)$ & \\
\hline $\mathbf{S i}$ & $-2.58 \pm 0.13$ & $-2.43 \pm 0.32$ & $-2.53 \pm 0.22$ \\
\hline PIV & - & $-4.33 \pm 0.27$ & \\
\hline $\mathrm{Pv}_{\mathrm{v}}$ & - & $-4.01 \pm 0.12$ & \\
\hline $\mathbf{P}$ & - & $-4.12 \pm 0.22$ & $-4.12 \pm 0.22$ \\
\hline $\mathrm{S}_{\text {III }}$ & $-3.38 \pm 0.14$ & - & \\
\hline S IV & - & $-3.18 \pm 0.21$ & \\
\hline $\mathrm{S}_{\mathrm{V}}$ & - & $-2.77 \pm 0.19$ & \\
\hline S vI & - & $(-2.69 \pm 1.03)$ & \\
\hline $\mathbf{S}$ & $-3.38 \pm 0.17$ & $-2.94 \pm 0.22$ & $-3.18 \pm 0.20$ \\
\hline $\mathrm{Fe}$ & - & $-2.68 \pm 0.25$ & $-2.68 \pm 0.25$ \\
\hline $\mathbf{N i}$ & - & $-3.11 \pm 0.26$ & $-3.11 \pm 0.26$ \\
\hline
\end{tabular}

for $\mathrm{C}, \mathrm{N}, \mathrm{O}, \mathrm{Ne}, \mathrm{Mg}, \mathrm{Al}, \mathrm{Si}, \mathrm{P}, \mathrm{S}, \mathrm{Fe}$, and Ni. Tables 7 and 8 lists the resulting number abundances ratios with respect to hydrogen.

\subsubsection{Carbon, nitrogen, and oxygen}

There are plenty of lines both in the FUV and the visual for carbon and nitrogen in the C\&N-type stars [CW83] 0904-02 LSS 1274 , and LS IV $+10^{\circ} 9$. However, only a few carbon lines are found in the CD- $31^{\circ} 4800$ spectra. Fits to selected carbon lines of different ionization stages are displayed in Fig. 5 for LSS 1274. C II $4267 \AA$ may be an important spectroscopic diagnostic when observed in such hot stars. The line is faintly present in the spectra of the C-rich stars, but not in the C-poor CD$31^{\circ} 4800$ (see Fig. A. 3 in the appendix).

For CD-31 4800 the many visual and FUV nitrogen lines are even stronger than for the former stars. Both elements are present in several stages of ionization. In particular, nitrogen lines from $\mathrm{N}_{\text {II, }} \mathrm{N}$ III, and $\mathrm{N}$ IV are present. Figure 5 illustrates the match of synthetic spectra to observations for a few spectral lines for LSS 1274. The final model spectra reproduce the N III and N IV as well as the $\mathrm{C}_{\mathrm{III}}$ and $\mathrm{C}$ IV lines very well, indicating that their ionization equilibria are matched and the effective temperature is consistent.

\begin{tabular}{|c|c|c|c|}
\hline \multicolumn{4}{|c|}{$\mathrm{LS} \mathrm{IV}+10^{\circ} 9$} \\
\hline Element & Visual/UVA & FUSE & Adopted \\
\hline $\mathrm{He}_{\text {I }} \& \mathrm{He}_{\text {II }}$ & $2.73 \pm 0.25$ & - & $2.73 \pm 0.25$ \\
\hline $\mathrm{C}_{\text {III }}$ & $0.17 \pm 0.03$ & $0.12 \pm 0.10$ & \\
\hline C IV & - & $0.09 \pm 0.15$ & \\
\hline $\mathbf{C}$ & $0.17 \pm 0.10$ & $0.11 \pm 0.16$ & $0.15 \pm 0.13$ \\
\hline $\mathrm{N}_{\text {II }}$ & $-0.01 \pm 0.04$ & - & \\
\hline $\mathrm{N}_{\text {III }}$ & $-0.13 \pm 0.21$ & $-0.18 \pm 0.21$ & \\
\hline $\mathrm{N}_{\text {IV }}$ & $-0.21 \pm 0.07$ & $-0.33 \pm 0.47$ & \\
\hline $\mathbf{N}$ & $-0.09 \pm 0.19$ & $-0.22 \pm 0.36$ & $-0.13 \pm 0.27$ \\
\hline $\mathrm{O}_{\text {II }}$ & $-0.70 \pm 0.03$ & - & \\
\hline $\mathrm{O}_{\text {III }}$ & $-0.75 \pm 0.06$ & $-0.81 \pm 0.05$ & \\
\hline O IV & - & $-0.89 \pm 0.26$ & \\
\hline $\mathbf{O}$ & $-0.72 \pm 0.11$ & $-0.82 \pm 0.18$ & $-0.76 \pm 0.15$ \\
\hline $\mathrm{Ne}_{\text {II }}$ & $0.07 \pm 0.02$ & $0.25 \pm 0.31$ & \\
\hline $\mathrm{Ne}$ III & - & $0.15 \pm 0.27$ & \\
\hline $\mathrm{Ne}$ & $0.07 \pm 0.10$ & $0.17 \pm 0.31$ & $0.09 \pm 0.21$ \\
\hline $\mathrm{Mg}_{\text {II }}$ & $-1.21 \pm 0.10$ & - & $-1.21 \pm 0.10$ \\
\hline Al III & $-2.13 \pm 0.10$ & $(-2.21 \pm 0.60)$ & $-2.13 \pm 0.10$ \\
\hline Si III & $-1.21 \pm 0.06$ & $-1.07 \pm 0.14$ & \\
\hline Si IV & $-1.17 \pm 0.03$ & $-0.96 \pm 0.24$ & \\
\hline $\mathbf{S i}$ & $-1.18 \pm 0.11$ & $-1.03 \pm 0.21$ & $-1.12 \pm 0.16$ \\
\hline PIV & $-2.87 \pm 0.10$ & $-3.40 \pm 0.15$ & \\
\hline $\mathrm{PV}_{\mathrm{V}}$ & - & $-3.02 \pm 0.35$ & \\
\hline $\mathbf{P}$ & $-2.87 \pm 0.15$ & $-3.29 \pm 0.27$ & $-3.02 \pm 0.21$ \\
\hline $\mathrm{S}_{\text {III }}$ & $(-3.53 \pm 0.87)$ & - & \\
\hline $\mathrm{S}_{\text {IV }}$ & - & $-2.18 \pm 0.19$ & \\
\hline $\mathrm{Sv}$ & - & $-1.91 \pm 0.19$ & \\
\hline S vI & - & $(-2.37 \pm 0.85)$ & \\
\hline $\mathbf{S}$ & $(-3.53 \pm 0.87)$ & $-2.05 \pm 0.21$ & $-2.05 \pm 0.21$ \\
\hline $\mathrm{Fe}$ & - & $-1.89 \pm 0.16$ & $-1.89 \pm 0.16$ \\
\hline $\mathbf{N i}$ & - & $-2.20 \pm 0.31$ & $-2.20 \pm 0.31$ \\
\hline
\end{tabular}

Oxygen lines are weak in all program stars. The $\mathrm{O}_{\text {II }}$ ion is observable only in CD-31 4800 . O III is available both in the FUV, in the UVA and visual spectrum (see Fig. 5). Accordingly, the oxygen abundances are low for all stars. It is worthwhile to note that the oxygen abundance of LS IV $+10^{\circ} 9$ is significantly higher than those derived for the other stars.

\subsubsection{Neon}

Many lines of neon (Ne II) lie in the UVA regime, which is difficult to observe from the ground. We take advantage of the capability of the UVES spectrograph to observe this spectral range. The Ne II line strength of the N-type He-sdO CD-31 4800 are considerably weaker than that of the $\mathrm{C}$-types, indicating quite different neon abundances. This is illustrated in Fig. 6, where the $\mathrm{Ne}$ II line fits for $\mathrm{CD}-31^{\circ} 4800$ are compared to those of LSS 1274 . While the neon abundance of CD-3 $1^{\circ} 4800$ turns out to be solar, it is about five times solar in LSS 1274 and even almost ten times in LS IV $+10^{\circ} 9$.

\subsubsection{Magnesium, aluminum, silicon, phosphorus, and sulfur}

In the visual spectrum the only magnesium line is $\mathrm{Mg}$ II $4481 \AA$. Since no Mg lines in the FUV could be used, the magnesium 
M. Schindewolf et al.: A quantitative NLTE analysis of four helium-rich subdwarf O stars
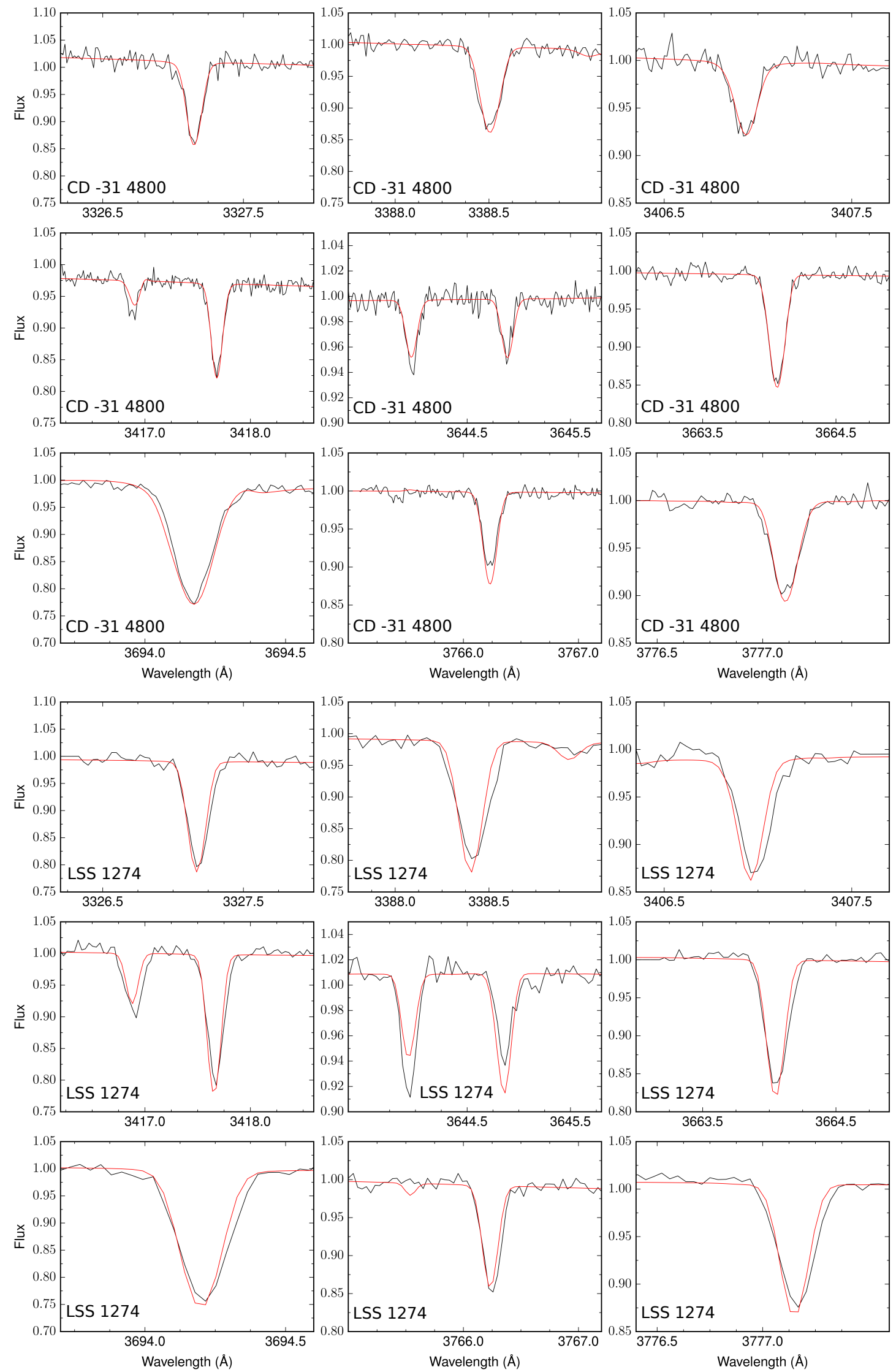

Fig. 6. Ne II lines: Comparison of the final synthetic spectrum to the normalized UVA spectrum. Upper half: CD-31 4800, lower half: LSS 1274 . We note that the strength of all lines are weaker in CD-31 4800 than in LSS 1274 because of the higher neon abundance. of the latter. 
Table 9. Logarithmic surface abundances by mass of the analyzed elements.

\begin{tabular}{cccccc}
\hline \hline Element & CD-31 ${ }^{\circ} 4800$ & LSS 1274 & [CW83] 0904-02 & LS IV +10 9 & Solar \\
\hline $\mathrm{H}$ & $-3.22 \pm 0.20$ & $-2.79 \pm 0.25$ & $-2.62 \pm 0.30$ & $-3.34 \pm 0.25$ & -0.13 \\
$\mathrm{He}$ & -0.0035 & -0.0138 & -0.0142 & -0.0121 & $-0.60 \pm 0.01$ \\
$\mathrm{C}$ & $-4.15 \pm 0.16$ & $-1.72 \pm 0.19$ & $-1.65 \pm 0.13$ & $-2.12 \pm 0.13$ & $-2.63 \pm 0.05$ \\
$\mathrm{~N}$ & $-2.38 \pm 0.11$ & $-2.75 \pm 0.22$ & $-3.05 \pm 0.19$ & $-2.33 \pm 0.27$ & $-3.16 \pm 0.04$ \\
$\mathrm{O}$ & $-4.22 \pm 0.13$ & $-3.41 \pm 0.24$ & $-3.47 \pm 0.22$ & $-2.90 \pm 0.15$ & $-2.24 \pm 0.05$ \\
$\mathrm{Ne}$ & $-2.74 \pm 0.12$ & $-2.19 \pm 0.21$ & $-2.28 \pm 0.16$ & $-1.95 \pm 0.21$ & $-2.90 \pm 0.10$ \\
$\mathrm{Mg}$ & $-3.39 \pm 0.10$ & $-3.36 \pm 0.29$ & $-3.64 \pm 0.16$ & $-3.17 \pm 0.10$ & $-3.15 \pm 0.05$ \\
$\mathrm{Al}$ & $-4.47 \pm 0.21$ & $-3.45 \pm 0.30$ & $-4.24 \pm 0.23$ & $-4.05 \pm 0.10$ & $-4.26 \pm 0.04$ \\
$\mathrm{Si}$ & $-3.47 \pm 0.16$ & $-3.50 \pm 0.19$ & $-3.70 \pm 0.22$ & $-3.02 \pm 0.16$ & $-3.18 \pm 0.03$ \\
$\mathrm{P}$ & $-5.61 \pm 0.15$ & $-5.10 \pm 0.15$ & $-5.25 \pm 0.22$ & $-4.88 \pm 0.21$ & $-5.24 \pm 0.03$ \\
$\mathrm{~S}$ & $-3.67 \pm 0.19$ & $-3.75 \pm 0.19$ & $-4.29 \pm 0.20$ & $-3.89 \pm 0.21$ & $-3.51 \pm 0.03$ \\
$\mathrm{Fe}$ & $-3.48 \pm 0.27$ & $-3.46 \pm 0.18$ & $-3.55 \pm 0.25$ & $-3.49 \pm 0.16$ & $-2.89 \pm 0.04$ \\
$\mathrm{Ni}$ & $-3.94 \pm 0.23$ & $-3.64 \pm 0.29$ & $-3.96 \pm 0.26$ & $-3.78 \pm 0.31$ & $-4.15 \pm 0.04$ \\
\hline
\end{tabular}

Notes. The values have been derived from Table 7. Hydrogen was the reference element in the calculations of the model atmospheres. The last column gives the solar mass abundances taken from Asplund et al. (2009) as a reference.

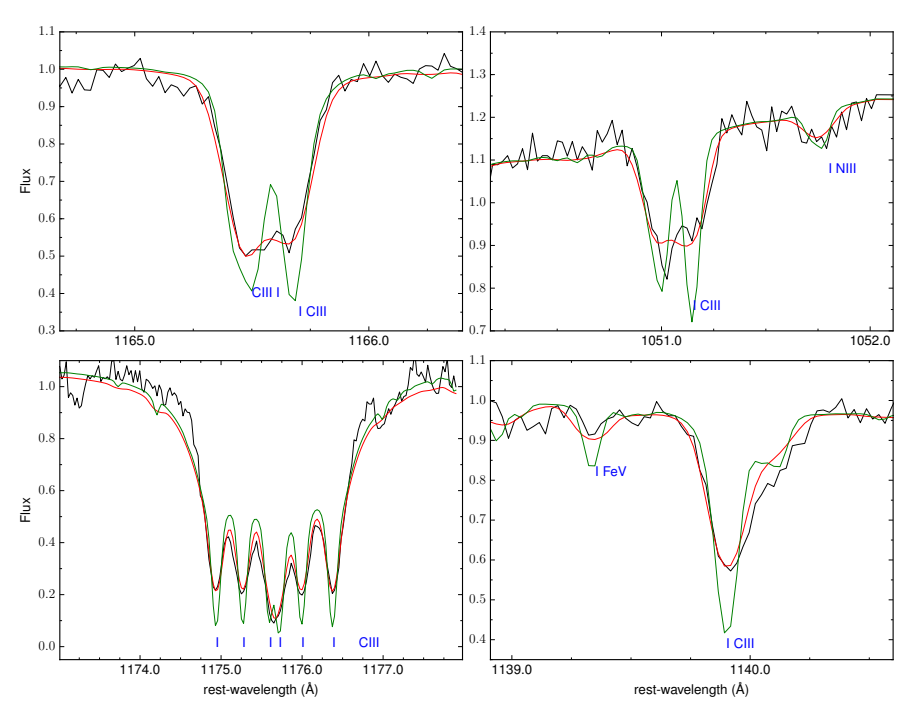

Fig. 7. Fits to $\mathrm{C}_{\text {III }}$ lines in the FUSE FUV spectrum of [CW83] 0904-02. The red profile is calculated with the derived projected rotational velocity of $30 \mathrm{~km} \mathrm{~s}^{-1}$. The green line indicates the line profiles without rotation for comparison. As in the case of LSS 1274 the continuum adjustment for the CIII septuplet at $1176 \AA$ is problematic (see Fig. 5).

abundance is based on a single line, which, however, is well reproduced by the final synthetic spectra in all program stars. For aluminum, the lines at $3601.63 \AA$ and $5696.6 \AA$ of $\mathrm{Al}$ III were visible in all program stars, but no other ionization stage is observable. The FUV supplements two additional $\mathrm{Al}$ III lines (see Table A.5).

A variety of lines of silicon can be found in the visual, the $\mathrm{Si}$ III lines being faint or absent in the hotter stars. Additional Si lines in the FUV were used for all program stars. As for carbon and nitrogen, we find $\mathrm{Si}$ III and $\mathrm{Si}$ Iv lines to be matched very well by the spectral line fit, indicating that the effective temperature is correct. All visual phosphorus lines were relatively weak and only usable in some of the sample stars. The strong phosphorus lines located in the FUSE range are the best choice to derive the phosphorus abundance. Both $\mathrm{P} I V$ and $\mathrm{P} v$ are matched well. The same holds for sulfur, for which the FUV lines of S IV and S v are the best choice.

\subsubsection{Iron and nickel}

The iron group elements dominate the ultraviolet spectral range of the program stars. We concentrate on the iron and nickel lines for three and four times ionized species. In Table A.5 the selected iron and nickel lines are listed. Some lines are given as single lines as well as part of a wider fit window with multiple lines. Some lines listed as multiplets in Table A.5, cannot be resolved in single lines. Iron and nickel lines were fitted in the same way as spectral lines from other chemical elements.

\subsection{Elemental composition of the program stars}

In Table 9 the elemental composition of the atmospheres of the program stars are summarized as mass fractions and compared to the solar composition (Asplund et al. 2009). As expected for He-sdOs, helium is dominant (97-99\% by mass), hydrogen is strongly depleted in all stars, in fact only $0.06 \%-0.2 \%$ of the atmospheric mass is hydrogen and carbon, nitrogen, or neon may be more abundant than hydrogen. The carbon mass fractions range from $1 \%-2 \%$ in the $\mathrm{C}$-rich stars, while nitrogen is the second most abundant element $(0.4 \%)$ in CD-31 4800 . Neon provides $1 \%$ of the atmospheric mass for $\operatorname{LSIV}+10^{\circ} 9$. The abundance pattern of CD-31 4800 is shown in Fig. 8 and compared to those of the other three program stars in Fig. 8.

In CD-31 4800 , a clear CNO bi-cycle abundance pattern is observed. Nitrogen is enriched, while carbon and oxygen are depleted. Neon is slightly above solar, while the other intermediate mass elements are below solar by a factor of about 2 . Iron is depleted compared to the Sun while Ni is slightly above solar.

LSS 1274, LS IV $+10^{\circ}$ 9, and [CW83] 0904-02 are enriched in carbon with respect to the sun, while oxygen is depleted in all three stars. LS IV $+10^{\circ} 9$ is as strongly enriched in nitrogen as is CD-31 ${ }^{\circ} 4800$, while LSS 1274 is somewhat less enriched than CD-31 4800 . The nitrogen abundance of [CW83] 0904-02 is close to solar. The carbon-to-nitrogen ratio by mass is $X_{\mathrm{C}} / X_{\mathrm{N}}=1.7$ for $\mathrm{LS} \mathrm{IV}+10^{\circ} 9,11$ for LSS 1274, and 25 for [CW83] 0904-02. Neon is enriched with respect to the Sun in LSS 1274, [CW83] 0904-02, and LS IV $+10^{\circ} 9$ by factors between three and ten, while the other intermediate mass elements all scatter around the solar abundance level. In general, the abundances in LS IV $+10^{\circ} 9$ seem to be a little bit higher 

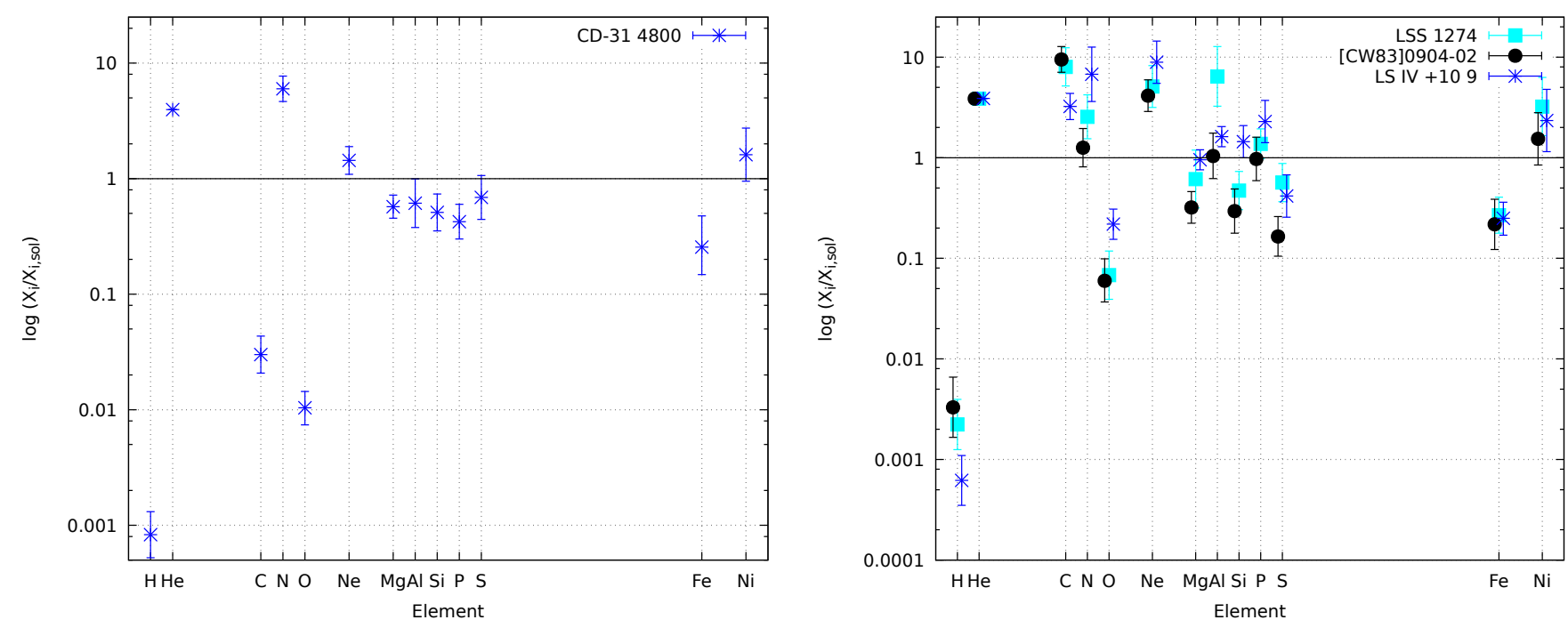

Fig. 8. Abundance pattern of CD-31 4800 (left hand panel) and LSS 1274, [CW83] 0904-02, and LS IV $+10^{\circ} 9$ (right hand panel) with respect to solar composition.

when compared to the other two stars. While iron is subsolar, the level of nickel is solar in LS IV $+10^{\circ} 9$ and in [CW83] 0904-02 and supersolar in LSS 1274.

\subsection{Projected rotation velocity}

CD-31 4800 , LSS 1274 and LS IV $+10^{\circ} 9$ show no rotational broadening. For these stars, the projected rotational velocity is lower than $v_{\text {rot }} \sin i=5 \mathrm{~km} \mathrm{~s}^{-1}$, the detection limit. Only for [CW83] 0904-02 rotational line broadening is detected. The projected rotation velocity was measured from the carbon lines in the visual spectrum of [CW83] 0904-02 to be $v_{\text {rot }} \sin i=$ $35 \pm 4 \mathrm{~km} \mathrm{~s}^{-1}$ (see Fig. A.1). The rotational broadening is also obvious in the FUSE FUV spectrum (see Fig. 7) and was derived to be $30 \pm 4 \mathrm{~km} \mathrm{~s}^{-1}$. Hence the projected rotational velocity from the FUV is consistent with the visual one within error margins.

\section{Evolutionary status: Mergers vs. flashers}

Confronting the properties of the program stars with evolutionary models should allow their origin to be constrained. We introduce the two rivalling scenarios and summarize the main observational results, before discussing the scenarios in depth.

Two rivaling scenarios have been proposed to explain the origin of He-sdO stars, the helium WD merger (Webbink 1984) and the late hot flasher scenario (Sweigart 1997; Miller Bertolami et al. 2008). In the merger scenario a binary system consisting of two He WDs loses orbital energy and angular momentum by the emission of gravitational waves and the orbit shrinks until the lighter component overfills its Roche lobe and disrupts, forming an accretion disk and possibly a hot corona around the more massive component. Once this material has been accreted by the massive He WD, helium is ignited in the core and the remnant emerges as a hot subluminous star. The surface composition may be altered by thermonuclear reactions and mixing during the accretion process. Depending on the initial masses of the stars and the rate of accretion, thermonuclear processes may occur and change the chemical composition of the companion (Zhang \& Jeffery 2012).

The late hot flasher scenario does not require binary evolution and can also happen in single stars. If a low-mass star loses a sufficient amount of material on the red giant branch (RGB) before the helium core flash occurs, a delayed core helium flash kicks in when the star descends along the hot WD cooling track (Castellani \& Castellani 1993). Hot subdwarfs formed via this scenario can be found at the hot end of the extreme horizontal branch, close to the helium main sequence. The depletion of hydrogen can be explained by the presence of a sub-surface convection zone emerging during the helium flash that pushes hydrogen into deeper layers where it is diluted and can be burnt. Depending on the epoch when the core flash occurs, that means on or before reaching the WD cooling sequence, theoretical models predict different abundance patters to emerge in the stellar atmospheres (Brown et al. 2001; Cassisi et al. 2003; Lanz et al. 2004).

The main observational results can be used to narrow down the parameter space for the evolutionary models:

1. The abundance patterns:

- Hydrogen is a trace element and detected at a mass fraction of $0.06 \%-0.2 \%$.

- The overall metallicity of the stars is solar or slightly below solar (factor 2). Metal poor models can, therefore, be discarded.

- Signatures of nucleosynthesis are obvious for C, N, O, and $\mathrm{Ne}$.

- CD-31 4800 displays $\mathrm{C}$ and $\mathrm{O}$ depletion and, N enrichment with respect to the Sun characteristic for processing by the CNO bicycle (see Fig. 8, left hand panel).

- All C-rich stars have enhanced C, N, and Ne, while O is depleted with respect to the Sun (see Fig. 8, right hand panel).

2. Abundance ratios for $\mathrm{N} / \mathrm{O}$ and $\mathrm{N} / \mathrm{Ne}$ show a trend to increase with increasing N/C (Fig. 9) for the four stars.

3. The stellar masses are consistent with the canonical mass of $0.47 M_{\odot}$, which is in line with all variants of hot flasher models.

- [CW83] 0904-02 is possibly more massive $\left(\approx 0.6 M_{\odot}\right)$, while the others are somewhat lighter $\left(\approx 0.4 M_{\odot}\right)$.

- CD-31 4800 , LSS 1274 , and LS IV $+10^{\circ} 9$ may have formed from mergers of pairs of low mass He WD $\left(0.15-0.35 M_{\odot}\right)$, that is their progenitors could involve at least one He WD of extremely low mass (ELM, $M<$ $0.2 M_{\odot}$, see Heber 2017), while [CW83] 0904-02 could result from normal He WDs. 

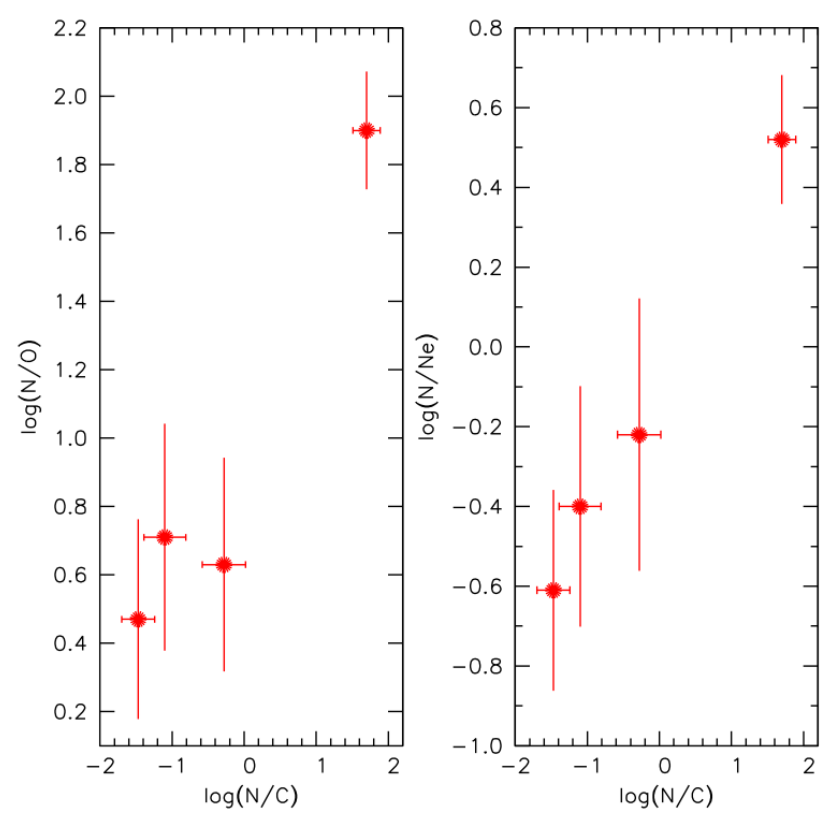

Fig. 9. Abundance ratios by number: N/O versus N/C (left hand panel) and $\mathrm{N} / \mathrm{Ne}$ vs. N/C (right hand panel).

- [CW83] 0904-02 could be a merger from more "normal mass" He WDs.

4. Rotation: Projected rotation velocities are below the detection limit $\left(5 \mathrm{~km} \mathrm{~s}^{-1}\right)$ except for [CW83] 0904-02, which rotates at $v_{\text {rot }} \sin i=30 \mathrm{~km} \mathrm{~s}^{-1}$.

\subsection{Formation through He-WD mergers}

Stellar mergers of two He WDs have been suggested to explain the origin of He-sdO and -sdB stars. Zhang \& Jeffery (2012) modelled the post-merger evolution of a helium plus helium double WD for four equal mass pairs $\left(0.25 M_{\odot}+0.25 M_{\odot} ; 0.3 M_{\odot}+\right.$ $0.3 M_{\odot} ; 0.35 M_{\odot}+0.35 M_{\odot} ; 0.4 M_{\odot}+0.4 M_{\odot}$ ) in 1D geometry. The result of the merging process depends on the mass transfer rate. In the slow merger model, a debris disk forms from which the primary WD accretes. Temperatures are too low to allow nuclear burning to take place. The material accumulating at the surface of the primary is that of the disrupted He WD, that is rich in helium and nitrogen, but poor in $\mathrm{C}$ and eventually oxygen. If the mass transfer rate is high the less massive WD quickly transfers its entire mass to the companion's surface directly. The heating forces the material to expand and a hot corona quickly builds up. The high temperatures enable helium burning and a strong convection zone forms to possibly dredge up fresh carbon to the surface. The efficiency of the dredge up increases with merger mass. The third model investigated by Zhang \& Jeffery (2012) is a composite one assuming both processes to occur. The surface abundance pattern from a composite merger should be a mix of the patterns of both slow and fast models. High-mass mergers models predict carbon-rich material to be dredged-up, whereas low-mass ones do not. Accordingly, the composite merger models of Zhang \& Jeffery (2012) predict the atmospheres to become $\mathrm{C}$-rich if the mass of the merger remnant exceeds $0.65 M_{\odot}$, but to be N-rich for lower masses.

The N-rich, C- and O-poor composition of CD-31 4800 (Fig. 8) resembles that of a helium WD, that is the core composition of a RGB star, as demonstrated in Table 10. The abundances predicted for the typical composition of the He-cores of
Table 10. Comparison of the abundances (logarithmic mass fractions) derived for CD-31 4800 to the typical composition of the He-cores of RGB stars (of different mass $M_{\mathrm{He}-\text { core }}$ ), coming from progenitors of different initial masses ( $M_{\text {ZAMS }}$ ) and a metallicity of $z=0.01$ (as computed by Miller Bertolami 2016).

\begin{tabular}{lcccc}
\hline \hline & $\mathrm{C}$ & $\mathrm{N}$ & $\mathrm{O}$ & $\mathrm{Ne}$ \\
\hline $\mathrm{CD}-314800$ & $-4.14 \pm 0.16$ & $-2.38 \pm 0.11$ & $-4.22 \pm 0.13$ & $-2.74 \pm 0.12$ \\
& \multicolumn{2}{c}{$\mathrm{RGB}$ cores } \\
$M_{\mathrm{He}-\text { core }}\left(M_{\text {ZAMS }}\right)$ & $\mathrm{C}$ & $\mathrm{N}$ & $\mathrm{O}$ & $\mathrm{Ne}$ \\
\hline $0.20 M_{\odot}\left(1 M_{\odot}\right)$ & -4.30 & -2.22 & -3.13 & -3.01 \\
$0.45 M_{\odot}\left(1 M_{\odot}\right)$ & -4.20 & -2.20 & -3.39 & -3.01 \\
$0.20 M_{\odot}\left(1.5 M_{\odot}\right)$ & -4.17 & -2.19 & -3.53 & -3.01 \\
$0.45 M_{\odot}\left(1.5 M_{\odot}\right)$ & -4.13 & -2.19 & -3.69 & -3.01 \\
\hline
\end{tabular}

RGB stars of different masses $\left(M_{\mathrm{He}-\mathrm{core}}=0.2\right.$ and $\left.0.45 M_{\odot}\right)$ and from different initial masses $\left(1.0\right.$ and $\left.1.5 M_{\odot}\right)$ of the progenitors and the appropriate metallicity $(z=0.01$, Miller Bertolami 2016). The $\mathrm{C}, \mathrm{N}$, and $\mathrm{Ne}$ abundances of $\mathrm{CD}-31^{\circ} 4800$ are close to those predicted. However, for oxygen the model abundance is much higher than in CD- $31^{\circ} 4800$, which indicates that the NO- cycle might had been operating at higher temperatures in CD-31 4800 than in the models. Nevertheless, we conclude that the abundance of $\mathrm{CD}-31^{\circ} 4800$ are consistent with the slow (cold) merger model of a double He-WD binary. However, the He-sdO star resulting from such a merger would be expected to show some rotation (Németh et al. 2012a).

Indeed, Schwab (2018) predicts surface rotation of a 30 and $100 \mathrm{~km} \mathrm{~s}^{-1}$ for the merger remnant after merging He-WD binaries of $0.2+0.3 M_{\odot}$ and $0.3+0.4 M_{\odot}$, respectively. Because the projected rotation velocity of CD-3 $1^{\circ} 4800$ is small, the inclination of the rotation axis would need to be small if CD-31 ${ }^{\circ} 4800$ resulted from a He-WD merger.

The high $\mathrm{C}$ abundance in the other three stars can not be explained by a cold merger. However, hot and composite merger models may be appropriate. Figure 10 shows predictions for the abundances of $\mathrm{C}, \mathrm{N}, \mathrm{O}$, and $\mathrm{Ne}$ in a composite merger model as a function of the final merger mass and for solar metallicity $(z=0.02$, Zhang \& Jeffery 2012) and compares them to the abundances determined for [CW83] 0904-02. The carbon abundance is much too low for stellar masses of less than $0.7 M_{\odot}$, but comes close to the observed ones for more massive models. The same holds for neon, while the predicted $\mathrm{N}$ abundance is higher than the observed one for all masses, although it comes closer to the observed one for high masses. The predicted $\mathrm{O}$ abundance is much higher than the observed one as is the case for CD-31 4800 and the cold merger model prediction. For the composite merger the discrepancy for oxygen even increases with increasing mass. Hence, we conclude that the abundance pattern predicted by the composite merger models of Zhang \& Jeffery (2012) for equal mass HeWD mergers are inconsistent with the observed abundance patterns of the $\approx 0.6 M_{\odot}$ star [CW83] 0904-02 (Fig. 10). Because LSS 1274, and LS IV $+10^{\circ} 9$ are of lower mass, their abundances are differ strongly from the predictions. Additional calculations are required for pairs of unequal mass He-WDs. Threedimensional modelling would also be necessary for more robust predictions.

The only program star, for which we obtained a significant projected rotation velocity is [CW83] 0904-02 $\left(31 \mathrm{~km} \mathrm{~s}^{-1}\right)$, close to the prediction of the model for a $0.2+0.3 M_{\odot} \mathrm{He}$ WD merger of Schwab (2018). Therefore, the measured rotational velocity would point to the composite merger scenario for [CW83] 0904-02, as this is able to explain the observed rotation, 


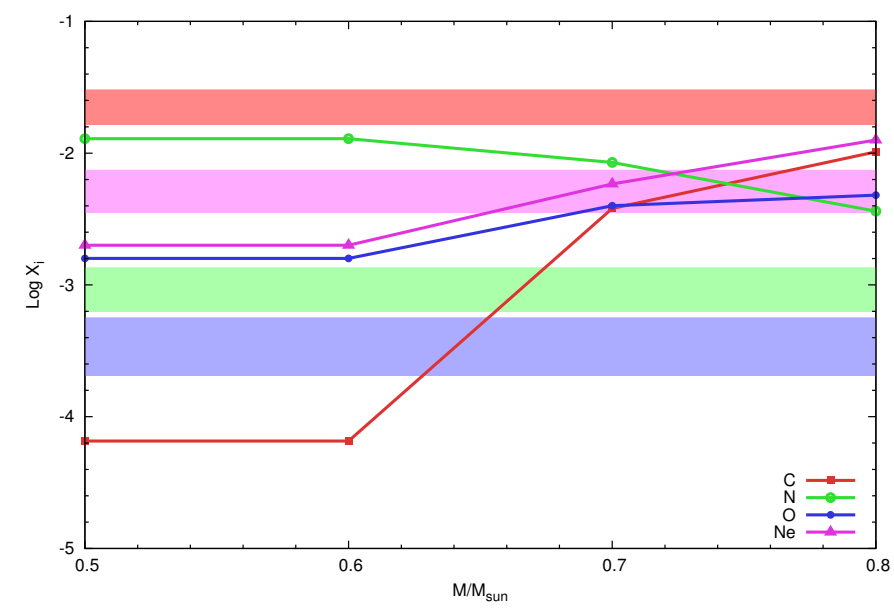

Fig. 10. Chemical abundances by mass for carbon $\left({ }^{12} \mathrm{C}+{ }^{13} \mathrm{C}\right)$, nitrogen $\left({ }^{14} \mathrm{~N}\right)$, oxygen $\left({ }^{16} \mathrm{O}+{ }^{18} \mathrm{O}\right)$, and neon $\left({ }^{20} \mathrm{Ne}+{ }^{22} \mathrm{Ne}\right)$ predicted by composite merger models (Zhang \& Jeffery 2012) as a function of the merger mass (solid colored lines) for a metallicity of $z=0.02$, The horizontal bands represent the abundances and their uncertainties determined for [CW83] 0904-02.

although its surface abundance is not fully consistent with the model prediction.

\subsection{Formation through late hot flashes}

The Helium core flash terminates the H-shell burning of red giant stars. In the canonical pictures the flash occurs at the tip of the RGB. However, the H-rich envelope may be lost before the onset of the He-core flash, for instance by binarity or enhanced winds due to stellar rotation (Sweigart 1997; Tailo et al. 2015) or the ingestion of a substellar companion (Soker 1998; Schaffenroth et al. 2014) or after a common envelope event (Byrne et al. 2018).

The remnant would then evolve toward high temperature and finally enter the WD cooling sequence. If a He-core flash occurs during this evolution, it is termed a hot flasher. Two types of hot flashers have to be considered, an early one, that occurs before the WD cooling sequence has been reached, and a late hot-flasher when the star is close or already on the WD cooling sequence. During the He-core flash a convective zone develops. In an early hot-flasher model, as in the canonical model, this zone does not reach the H-rich envelope and the surface abundances are not altered. Therefore, we can exclude early hotflasher to explain He-sdOs. However, in a late hot-flasher, which takes place when the star embarks on the WD cooling curve, the He-flash driven convective zone gets into contact with the $\mathrm{H}$ rich envelope. Depending on whether H-burning takes place or not, hot-flasher models are divided in deep-mixing and shallowmixing events. After the He-flash the surface becomes cooler because the stellar envelope expands triggering an outer convective zone to emerge which moves inwards. In the shallow mixing (SM) case the He-core flash driven convective zone eventually splits giving rise to a convective zone that persists in the outer region of the core until it merges with the outer convective zone of the envelope, which leads to a dredge up of material from the core to the surface. However, no H-burning occurs, but the $\mathrm{H}$ content at the surface decreases. In the deep mixing (DM) case, the convective region reaches the H-rich layer shortly after the He-flash maximum and $\mathrm{H}$ is mixed leading to violent $\mathrm{H}$-burning. As a consequence hydrogen almost vanishes from the

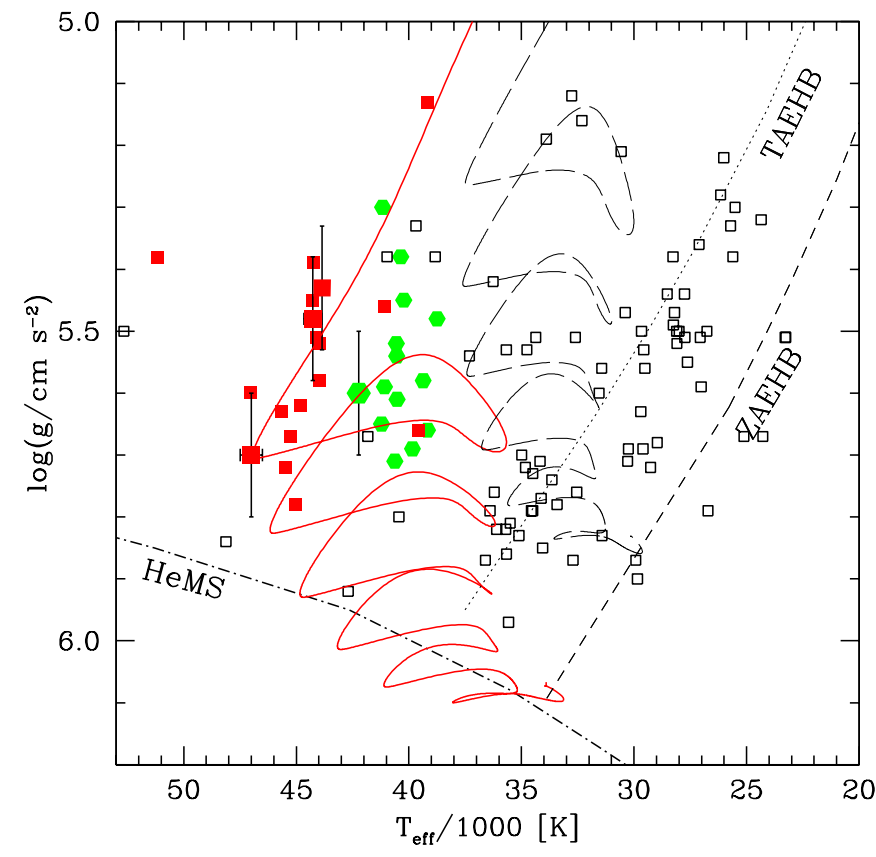

Fig. 11. Comparison of the positions of the program stars (plotted with error bars) in a Kiel diagram $\left(T_{\text {eff }}, \log g\right)$ with different evolutionary tracks for hot flashers (Battich et al. 2018) and the hot subdwarfs from the SPY project (Lisker et al. 2005; Hirsch 2009). The hydrogen-rich hot subdwarfs are marked by open squares, while C-rich $\mathrm{He}$-sdOs are are shown as red filled squares and $\mathrm{N}$-rich as green filled hexagons. The dashed (black) track is for a shallow mixing case and a stellar mass of $0.46794 M_{\odot}$, while the full drawn (red) one represent predictions from the deep-mixing hot flasher scenario for a mass of $0.46599 M_{\odot}$. Also shown are the zero- (ZAEHB) and terminal age EHB (TAEHB, adopted from Dorman et al. 1993) and the helium main sequence (Paczyński 1971).

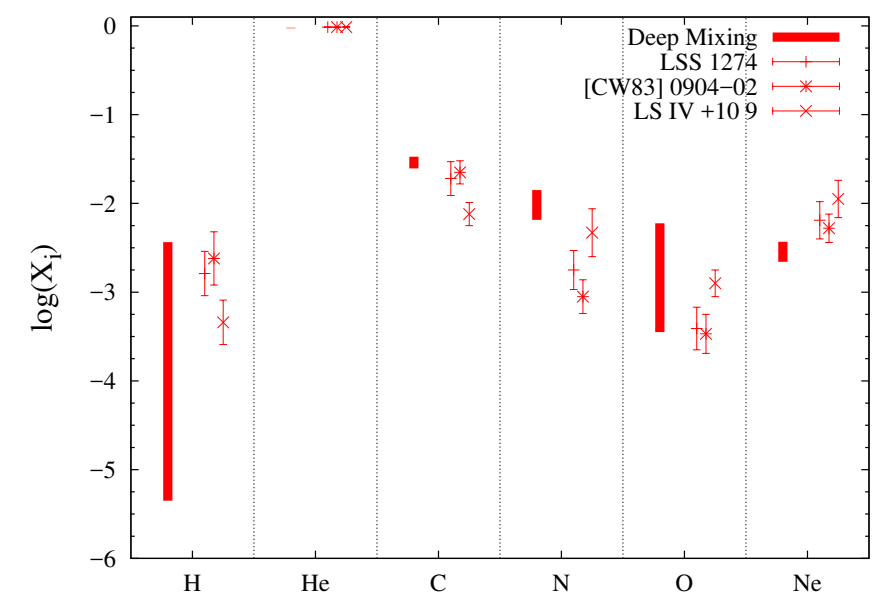

Fig. 12. $\mathrm{H}, \mathrm{He}, \mathrm{C}, \mathrm{N}, \mathrm{O}$ and $\mathrm{Ne}$ abundances of our stars as compared with the predictions from the deep-mixing late hot-flasher scenario (red bars, Battich et al. 2018).

surface and processed material is dredged up to the surface (see Battich et al. 2018, for details).

Figure 11 compares the positions of the program stars in the $T_{\text {eff }}-\log g$ diagram to evolutionary tracks of the hot flasher scenario with shallow and deep mixing. The shallow-mixing tracks are too cool to meet the observed positions and the remaining hydrogen content is too high. Shallow mixing can, therefore, be excluded. The tracks for deep mixing, however, evolve 


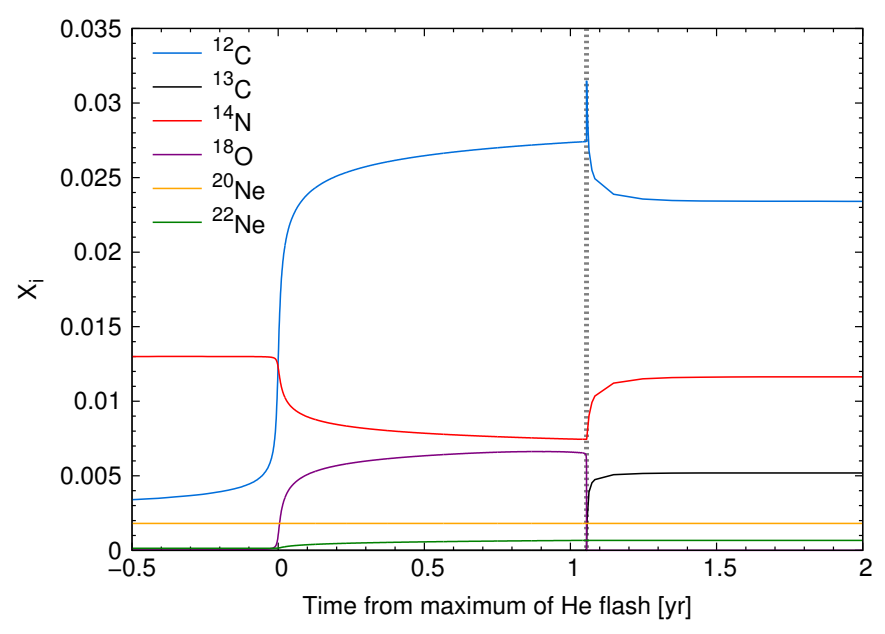

Fig. 13. Development of isotopic abundances during $\mathrm{He}-$ and $\mathrm{H}$-flashes. The abundances correspond to the inner part of the star where He- and $\mathrm{H}$-flashes take place. The dashed vertical (gray) line marks the onset of $\mathrm{H}$ flash. The abundances of ${ }^{16} \mathrm{O},{ }^{17} \mathrm{O}$ and ${ }^{15} \mathrm{~N}$ isotopes (not showed) are always below $5 \times 10^{-4}$.

through the region occupied by the program stars and, hence, are viable.

First, we compare in Fig. 12 the common pattern of hydrogen and light metal abundances of LSS 1274, [CW83] 0904-02 and LS IV $+10^{\circ} 9$ with those predicted by the stellar evolution simulations of Battich et al. (2018). In Fig. 12 we have chosen tracks with an initial metallicity and helium abundance of $z=0.02$ and $y=0.285$, respectively. The observed hydrogen abundance is at the high end of the prediction while the oxygen abundance is at the low one. The $\mathrm{C}$ and $\mathrm{N}$ abundances are slightly lower, while the $\mathrm{Ne}$ abundance is somewhat higher than predicted. Given the uncertainties involved in 1D evolutionary models we consider the observed abundance pattern to be consistent with the DM late flasher predictions. Qualitatively, the origin of the abundance pattern emerging from the flasher models of Battich et al. (2018) can be described as follows.

When the He flash starts ${ }^{14} \mathrm{~N}$ from the He-core is burnt with helium through the reactions

${ }_{7}^{14} \mathrm{~N}\left({ }_{2}^{4} \mathrm{He}, \gamma\right){ }_{9}^{18} \mathrm{~F}\left(e^{+} v_{e}\right){ }_{8}^{18} \mathrm{O}\left({ }_{2}^{4} \mathrm{He}, \gamma\right){ }_{10}^{22} \mathrm{Ne}$,

leading to a ${ }^{18} \mathrm{O}$ - and ${ }^{22} \mathrm{Ne}$-enrichment of the He-flash convective zone. Later, the $\mathrm{C}$ - and $\mathrm{Ne}$-rich $\mathrm{He}$-flash driven convective zone reaches the other, upper layers of the star, leading to a strong enrichment in both $\mathrm{C}$ and $\mathrm{Ne}$ at the photosphere. In addition, when this happens the remaining $\mathrm{H}$-envelope is mixed down into the hot He-burning interior, where it is burned with carbon. As a consequence the deep-mixing scenario is also expected to produce a strong $\mathrm{N}$-enrichment of the surface through the reactions

$$
{ }_{6}^{12} \mathrm{C}\left({ }_{1}^{1} \mathrm{H}, e^{+} v_{e}\right){ }_{6}^{13} \mathrm{C}\left({ }_{1}^{1} \mathrm{H}, \gamma\right){ }_{7}^{14} \mathrm{~N} \text {. }
$$

Fig. 13 shows the development of the abundances of the important elements between the He- and $\mathrm{H}$-flash in the simulation of a deep mixing event.

The helium flash takes place at $t=0$ and ${ }^{12} \mathrm{C}$ is significantly increased, at the expense of helium. ${ }^{18} \mathrm{O}$ and ${ }^{22} \mathrm{Ne}$ are enriched on the cost of ${ }^{14} \mathrm{~N}$. Shortly after about one year the hydrogen flash occurs and oxygen and ${ }^{12} \mathrm{C}$ is enriched for a short time before it is transformed to ${ }^{14} \mathrm{~N}$ by the reaction stated above. Immediately after the $\mathrm{H}$-flash the He-flash convective zone splits, with the outermost convective zone being driven by H-burning. Due to the lower temperatures of $\mathrm{H}$-burning the ${ }^{22} \mathrm{Ne}$ abundances do not change in the outermost convective region after the split. On the contrary, ${ }^{22} \mathrm{Ne}$ abundances in the innermost convective zone increase continuously during the He-flash, and consequently, the later the convective zone splits, the higher the final ${ }^{22} \mathrm{Ne}$ surface abundance of the models.

The correlation of abundance ratios in Fig. 9 might be interpreted in the context of hot-flasher models. ${ }^{22} \mathrm{Ne}$ is created at expenses of ${ }^{14} \mathrm{~N}$ at the same time that ${ }^{12} \mathrm{C}$ is created at expenses of ${ }^{4} \mathrm{He}$. This means that while the ${ }^{14} \mathrm{~N}$ abundance decreases, the ${ }^{12} \mathrm{C}$ and ${ }^{22} \mathrm{Ne}$ abundances increase, and naturally, that explains that $\mathrm{N} / \mathrm{Ne}$ correlates with $\mathrm{N} / \mathrm{C}$ in time. This correlation is present in the models in spite of the $\mathrm{N}$ created during the $\mathrm{H}$-flash (Battich et al. 2018). However, in the models too much $\mathrm{N}$ is produced during the $\mathrm{H}$-flash, while not enough ${ }^{22} \mathrm{Ne}$ is produced (see Fig. 12). This might indicate that the inner and outer convective zones during the Hot-Flasher event might stay connected longer than predicted by $1 \mathrm{D}$ stellar evolution models. The relatively low N/O values in our C-rich He-sdO stars can only be reproduced by hot-flasher models with a high ${ }^{18} \mathrm{O}$ abundance at the moment of the $\mathrm{H}$-flash. As before, the nitrogen-to-oxygen ratio of the models might also be improved if convective zones stay connected after the $\mathrm{H}$-flash.

The correlation between N/C-N/Ne and N/C-N/O may be telling that, for LSS 1274, [CW83] 0904-02 and LS IV +10 9, the material that appears in the surface was processed in a late deep-mixing He-flash event. Three-dimensional modelling would also be helpful for more robust predictions.

\section{Summary and conclusions}

We studied four prototypical He-sdO stars, the N-rich CD$31^{\circ} 4800$ and the C\&N-rich LSS 1274, LS IV $+10^{\circ} 9$, and [CW83] 0904-02 from high quality spectra using state-of-theart NLTE model atmosphere and spectral synthesis tools. The following results may be highlighted:

1. The analysis made use of data set of unprecedented quality, spectral resolution and coverage by combining ground-based visual and UVA data with FUV observations from the FUSE satellite.

2. Final models included $\mathrm{C}, \mathrm{N}, \mathrm{O}, \mathrm{Ne}, \mathrm{Mg}, \mathrm{Al}, \mathrm{Si}, \mathrm{S}, \mathrm{Fe}$, and $\mathrm{Ni}$ represented by the most detailed model atoms available. Because of the enrichment of either nitrogen or carbon, it turns out, that models including either $\mathrm{C}$ or $\mathrm{N}$ at the appropriate high abundance reproduce the temperature stratification of full models to within about $1 \%$ in the line forming regions. Therefore, derived atmospheric parameters differ by less than the observational uncertainties. Hence grids of synthetic spectra of $\mathrm{H} / \mathrm{He} / \mathrm{N}$ or $\mathrm{H} / \mathrm{He} / \mathrm{C}$ composition are sufficient to derive the atmospheric parameters of He-sdO stars. In order to avoid inconsistencies the chemical abundances are derived from full models at fixed effective temperatures and gravities by adding element by element in a sequence of decreasing solar abundance.

3. Chemical abundances:

Hydrogen is a trace element in all four program stars. Correlations among $\mathrm{C}, \mathrm{N}, \mathrm{O}$, and $\mathrm{Ne}$ abundances are found, that means the $\mathrm{O} / \mathrm{N}$ and $\mathrm{Ne} / \mathrm{N}$ ratios correlate positively with the $\mathrm{N} / \mathrm{C}$. For CD-31 4800 the He and N enrichment is accompanied by $\mathrm{C}$ and $\mathrm{O}$ depletion, a typical signature of hydrogen burning in the $\mathrm{CNO}$ bi-cycle. While the Ne abundance is solar. Neon is enriched in the C\&N-rich stars but shows a large scatter, extending up to a factor of ten with respect to 
the sun in LS IV $+10^{\circ} 9$.

Elements heavier than $\mathrm{Ne}$ are subsolar by about a factor of two, except for $\mathrm{Si}$ which is close to solar. The abundance patterns of the $\mathrm{C}$-rich He-sdOs are more complex. A somewhat subsolar metallicity is accompanied by $\mathrm{C}$ and $\mathrm{N}$-enrichment and O-deficiency, less pronounced than in CD-31 ${ }^{\circ} 4800$. The nickel-to-iron ratio is significantly supersolar.

4. Trigonometric parallaxes measured by the Gaia satellite allows us for the first time to derive stellar masses, which turns out to be consistent with predictions from canonical evolutionary models. From the analysis of spectral energy distributions we rule out the presence of late-type stellar companions.

5. The analyses aimed at constraining helium WD merger scenarios on the one hand and hot flasher scenarios on the other:

Merger Scenarios. The slow, cold merger slowly accreting from a disk, predicts no nuclear burning effecting the surface composition of the remnant. A fast merger predicts a hot corona to form and helium burning to occur. The composite merger scenario considers both processes to occur simultaneously. Except for the low oxygen abundance observed, the abundance pattern of CD-31 4800 is consistent with predictions of models of cold, slow helium WD mergers, that is the atmospheric composition is that of the interior of a lighter helium WD, that has been accreted by the more massive one to form the He-sdO. Carbon-rich He-sdOs can be formed in a composite merger, if their masses are significantly larger than the canonical one. Derived masses for LSS 1274 and $\operatorname{LSIV}+10^{\circ} 9$ are lower than the canonical value with [CW83] 0904-02 being the only relatively massive object of the sample. The remnant of a He-WD merger is expected to retain significant angular momentum and rotate at $30-100 \mathrm{~km} \mathrm{~s}^{-1}$. However, [CW83] 0904-02 is the only program star that rotates at the expected rate and may therefore be a viable merger remnant candidate.

Hot flasher scenarios. Early flasher as well as late flasher models with shallow mixing can be excluded because both predict much larger fractions of hydrogen to remain on the stellar surface. Therefore the late hot flasher scenario with deep mixing is the only viable one. The occurrence of convective zones predict the dredge-up of carbon during the helium flash as well as hydrogen burning during the mixing episodes. New evolutionary calculations predict abundance patterns similar to those determined for the $\mathrm{C}$ rich program stars. The C-deficient CD- $31^{\circ} 4800$, however, would be difficult to reconcile in the context of the flasher scenario.

In order to distinguish between the rivalling formation scenarios, it is of utmost importance to determine the stellar masses. The merger scenario predicts larger masses in particular for C-rich objects, exceeding about $0.65 M_{\odot}$. Hence, the masses derived from parallax and gravity may be tale telling. However, due to the uncertainties of the surfaces gravities the error margin is too large to be conclusive, although it is worthwhile to note that [CW83] 0904-02 is the most massive program star and, therefore, a promising candidate for a composite He-WD merger. The derived $\mathrm{C}, \mathrm{N}, \mathrm{O}$, and $\mathrm{Ne}$ abundances, in particular their correlations, provide important constraints for the merger models. A more accurate determination of the surface gravity will be needed to narrow down the uncertainties of the stellar masses, which are predominantly of systematic nature. Therefore, we started a project to obtain very high $\mathrm{S} / \mathrm{N}$, medium resolution spectra with very wide wavelength coverage for differential spectral analyses in order to reduce the systematic uncertainties of the surface gravities. In addition such differential quantitative spectral analyses have to be carried out for a larger sample to establish the mass distribution of He-sdO stars.

Acknowledgements. MS acknowledges funding by the Deutsche Zentrum für Luft- und Raumfahrt (grant No. 50 OR 1406). PN acknowledges funding by the Deutsche Forschungsgemeinschaft (grant No. HE 1356/45-2) and by the Grant Agency of the Czech Republic (grant No. GACR 18-20083S). This research has made use of ISIS functions provided by ECAP/Remeis obervatory. We thank Sabine Moehler for her help in reducing and correcting the UVES spectrum of CD-31 4800 . Some of the data presented in this paper were obtained from the Mikulski Archive for Space Telescopes (MAST). STScI is operated by the Association of Universities for Research in Astronomy, Inc., under NASA contract NAS5-26555. Support for MAST for non-HST data is provided by the NASA Office of Space Science via grant NNX09AF08G and by other grants and contracts. Based on observations at the Paranal Observatory of the European Southern Observatory for program number 69.C-0171(A), 072.D-0290(A), 077.C-0547(A) and 67.D-0047(A). Based on observations at the La Silla Observatory of the European Southern Observatory for programs number 074.B-0455. This research has used the services of Astroserver.org. TB and M3B are partially supported by ANPCyT through grant PICT-2014-2708, by the MinCyTDAAD bilateral cooperation program through grant DA/16/07, and by a Return Fellowship from the Alexander von Humboldt Foundation. M. L. acknowledges support from the Alexander von Humboldt Foundation.

\section{References}

Ahmad, A., \& Jeffery, C. S. 2003, A\&A, 402, 335

Asplund, M., Grevesse, N., Sauval, A. J., \& Scott, P. 2009, ARA\&A, 47, 481 Battich, T., Bertolami, M. M. M., Córsico, A. H., \& Althaus, L. G. 2018, A\&A, 614, A136

Bauer, F., \& Husfeld, D. 1995, A\&A, 300, 481

Berger, J., \& Fringant, A.-M. 1980, A\&A, 85, 367

Blair, W. P., Sankrit, R., Torres, S. I., Chayer, P., \& Danforth, C. W. 2009, ApJ, 692, 335

Blanchette, J.-P., Chayer, P., Wesemael, F., et al. 2008, ApJ, 678, 1329

Brown, T. M., Ferguson, H. C., Davidsen, A. F., \& Dorman, B. 1997, ApJ, 482, 685

Brown, T. M., Sweigart, A. V., Lanz, T., Landsman, W. B., \& Hubeny, I. 2001, ApJ, 562, 368

Byrne, C. M., Jeffery, C. S., Tout, C. A., \& Hu, H. 2018, MNRAS, 475, 4728

Cassisi, S., Schlattl, H., Salaris, M., \& Weiss, A. 2003, ApJ, 582, L43

Castellani, M., \& Castellani, V. 1993, ApJ, 407, 649

Chayer, P., Fontaine, M., Fontaine, G., Wesemael, F., \& Dupuis, J. 2006, Baltic Astron., 15, 131

Cutri, R. M. 2013, VizieR Online Data Catalog: II/328

Cutri, R. M., Skrutskie, M. F., van Dyk, S., et al. 2003, VizieR Online Data Catalog: II/246

Dekker, H., D’Odorico, S., Kaufer, A., Delabre, B., \& Kotzlowski, H. 2000, in Optical and IR Telescope Instrumentation and Detectors, eds. M. Iye, \& A. F. Moorwood, Proc. SPIE, 4008, 534

Dixon, W. V. D., Sankrit, R., \& Otte, B. 2006, ApJ, 647, 328

Dixon, W. V., Sahnow, D. J., Barrett, P. E., et al. 2007, PASP, 119, 527

Dorman, B., Rood, R. T., \& O'Connell, R. W. 1993, ApJ, 419, 596

Dreizler, S. 1993, A\&A, 273, 212

Dreizler, S., Heber, U., Werner, K., Moehler, S., \& de Boer, K. S. 1990, A\&A, 235,234

Drilling, J. S., \& Heber, U. 1987, in Second Conference on Faint Blue Stars, eds. A. G. D. Philip, D. S. Hayes, \& J. W. Heber, IAU Colloq., 95, 603

Edelmann, H., Heber, U., Hagen, H.-J., et al. 2003, A\&A, 400, 939

Ferraro, F. R., Paltrinieri, B., Fusi Pecci, F., et al. 1997, ApJ, 484, L145

Fitzpatrick, E. L. 1999, PASP, 111, 63

Fontaine, M., Chayer, P., Wesemael, F., Fontaine, G., \& Lamontagne, R. 2006, Baltic Astron., 15, 99

Fontaine, G., Green, E., Brassard, P., Latour, M., \& Chayer, P. 2014, in 6th Meeting on Hot Subdwarf Stars and Related Objects, eds. V. van Grootel, E. Green, G. Fontaine, \& S. Charpinet, Astronomical Society of the Pacific Conference Series, 481, 83

Friedman, S. D., Howk, J. C., Chayer, P., et al. 2002, ApJS, 140, 37

Gaia Collaboration (Brown, A. G. A., et al.) 2018, A\&A, 616, A1

Garrison, R. F., \& Hiltner, W. A. 1973, ApJ, 179, L117

Geier, S. 2013, A\&A, 549, A110

Giddings, J. R. 1981, PhD Thesis, University of London

Green, G. M., Schlafly, E. F., Finkbeiner, D., et al. 2018, MNRAS, 478, 651 Han, Z., Podsiadlowski, P., \& Lynas-Gray, A. E. 2007, MNRAS, 380, 1098

Heber, U. 2009, ARA\&A, 47, 211

Heber, U. 2016, PASP, 128, 082001 
Heber, U. 2017, in 20th European White Dwarf Workshop, eds. P.-E. Tremblay, B. Gaensicke, \& T. Marsh, Astronomical Society of the Pacific Conference Series, 509, 85

Heber, U., Irrgang, A., \& Schaffenroth, J. 2018, Open Astron., 27, 35

Hébrard, G., \& Moos, H. W. 2003, ApJ, 599, 297

Henden, A. A., Templeton, M., Terrell, D., et al. 2016, VizieR Online Data Catalog: II $/ 336$

Hirsch, H. A. 2009, PhD Thesis, Friedrich-Alexander University ErlangenNürnberg

Høg, E., Fabricius, C., Makarov, V. V., et al. 2000, A\&A, 355, L27

Hubeny, I., \& Lanz, T. 1995, ApJ, 439, 875

Hubeny, I., \& Lanz, T. 2017a, ArXiv e-prints [arXiv:1706.01859]

Hubeny, I., \& Lanz, T. 2017b, ArXiv e-prints [arXiv:1706.01935]

Hubeny, I., \& Lanz, T. 2017c, ArXiv e-prints [arXiv:1706.01937]

Hubeny, I., Lanz, T., \& Jeffery, C. 1994, Newsletter on Analysis of Astronomical Spectra, ed. C. S. Jeffery, Report, St. Andrews University, 20, 30

Husfeld, D., Butler, K., Heber, U., \& Drilling, J. S. 1989, A\&A, 222, 150

Jenkins, E. B. 2013, ApJ, 764, 25

Kaufer, A., Stahl, O., Tubbesing, S., et al. 1999, The Messenger, 95, 8

Kilkenny, D., \& Muller, S. 1989, S. Afr. Astron. Obs. Circular, 13, 69

Kramida, A., Ralchenko, Y., Reader, J., \& NIST ASD Team 2018, NIST Atomic Spectra Database (version 5.5.3), [Online]. Available: https://physics. nist.gov/asd [Tue Mar 20 2018], Report, National Institute of Standards and Technology, Gaithersburg, MD

Lallement, R., Hébrard, G., \& Welsh, B. Y. 2008, A\&A, 481, 381

Landolt, A. U. 2007, AJ, 133, 2502

Lanz, T., Hubeny, I., \& Heap, S. R. 1997, ApJ, 485, 843

Lanz, T., Brown, T. M., Sweigart, A. V., Hubeny, I., \& Landsman, W. B. 2004, ApJ, 602, 342

Latour, M., Fontaine, G., Chayer, P., \& Brassard, P. 2013, ApJ, 773, 84

Latour, M., Chayer, P., Green, E. M., Irrgang, A., \& Fontaine, G. 2018, A\&A, 609, A89

Lawrence, A., Warren, S. J., Almaini, O., et al. 2007, MNRAS, 379, 1599

Lisker, T., Heber, U., Napiwotzki, R., et al. 2005, A\&A, 430, 223

Mermilliod, J.-C., Mermilliod, M., \& Hauck, B. 1997, A\&AS, 124

Miller Bertolami, M. M. 2016, A\&A, 588, A25

Miller Bertolami, M. M., Althaus, L. G., Unglaub, K., \& Weiss, A. 2008, A\&A, 491,253

Moos, H. W., Cash, W. C., Cowie, L. L., et al. 2000, ApJ, 538, L1
Morton, D. C. 2003, ApJS, 149, 205

Morton, D. C., \& Dinerstein, H. L. 1976, ApJ, 204,

Napiwotzki, R. 1999, A\&A, 350, 101

Napiwotzki, R. 2008, in Hot Subdwarf Stars and Related Objects, eds. U. Heber, C. S. Jeffery, \& R. Napiwotzki, Astronomical Society of the Pacific Conference Series, 392, 139

Napiwotzki, R., Yungelson, L., Nelemans, G., et al. 2004, in Spectroscopically and Spatially Resolving the Components of the Close Binary Stars, eds. R.W. Hilditch, H. Hensberge, \& K. Pavlovski, Astronomical Society of the Pacific Conference Series, 318, 402

Naslim, N., Jeffery, C. S., Ahmad, A., Behara, N. T., \& Şahìn, T. 2010, MNRAS, 409, 582

Németh, P., Kawka, A., \& Vennes, S. 2012a, MNRAS, 427, 2180

Németh, P., Kawka, A., \& Vennes, S., 2012b, in Fifth Meeting on Hot Subdwarf Stars and Related Objects, eds. D. Kilkenny, C. S. Jeffery, \& C. Koen, Astronomical Society of the Pacific Conference Series, 452, 33

Ohl, R. G., Chayer, P., \& Moos, H. W. 2000, ApJ, 538, L95

Paczyński, B. 1971, Acta Astron., 21, 1

Paunzen, E. 2015, A\&A, 580, A23

Rauch, T., Rudkowski, A., Kampka, D., et al. 2014, A\&A, 566, A3

Sahnow, D. J., Moos, H. W., Ake, T. B., et al. 2000, ApJ, 538, L7

Schaffenroth, V., Classen, L., Nagel, K., et al. 2014, A\&A, 570, A70

Schlafly, E. F., \& Finkbeiner, D. P. 2011a, ApJ, 737, 103

Schlafly, E. F., \& Finkbeiner, D. P. 2011b, ApJ, 737, 103

Schwab, J. 2018, MNRAS, 476, 5303

Skrutskie, M. F., Cutri, R. M., Stiening, R., et al. 2006, AJ, 131, 1163

Soker, N. 1998, AJ, 116, 1308

Sonneborn, G., André, M., Oliveira, C., et al. 2002, ApJS, 140, 51

Stroeer, A., Heber, U., Lisker, T., et al. 2007, A\&A, 462, 269

Sweigart, A. V. 1997, in The Third Conference on Faint Blue Stars, eds. A. G. D. Philip, J. Liebert, R. Saffer, \& D. S. Hayes, 3

Tailo, M., D’Antona, F., Vesperini, E., et al. 2015, Nature, 523, 318

Ulla, A., \& Thejll, P. 1998, A\&AS, 132, 1

Walker, A. R. 1981, MNRAS, 197, 241

Webbink, R. F. 1984, ApJ, 277, 355

Werner, K., \& Dreizler, S. 1999, J. Comput. Appl. Math., 109, 65

Wolf, C., Onken, C. A., Luvaul, L. C., et al. 2018, PASA, 35, e010

Wood, B. E., Linsky, J. L., Hébrard, G., et al. 2004, ApJ, 609, 838

Zhang, X., \& Jeffery, C. S. 2012, MNRAS, 419, 452 


\section{Appendix A: Additional material}

In this appendix we give details about the spectroscopic data used (Sect. A.1), list the lines used for the determination of the atmospheric parameters in Sect. A.2 and illustrate the quality of fit for [CW83] 0904-02 in Sect. A.3. We present a reanalysis of CASPEC spectra in Sect. A.4 and show the SED fits for [CW83] 0904-02 and LSS 1274 in Sect. A.6. Finally, we list the metal lines used for the abundance analyses in Sect. A.7.

\section{A.1. Spectroscopic data}

Table A.1 lists the observational data for the four program stars with the available information.

\section{A.2. Hydrogen and helium lines}

Hydrogen and helium lines used to obtain the atmospheric parameters are listed in Table A.2.

Table A.1. List of observational data for the four He-sdO stars.

\begin{tabular}{|c|c|c|c|c|c|}
\hline$\underline{\text { Star }}$ & Instrument & Program ID & Wavelength range $(\AA)$ & Exposure time (s) & $\underline{\mathrm{R}}$ \\
\hline $\mathrm{CD}-31^{\circ} 4800$ & UVES & 69.C-0171(A) & $3281-4562$ & 2700 & 68642 \\
\hline $\mathrm{CD}-31^{\circ} 4800$ & UVES & 69.C-0171(A) & $4583-6686$ & 2700 & 107200 \\
\hline $\mathrm{CD}-31^{\circ} 4800$ & FEROS & 074.B-0455(A) & $3527 \ldots 9216$ & 600 & 48000 \\
\hline $\mathrm{CD}-31^{\circ} 4800$ & FUSE & p2050603000 & 905-1185 & 11747 & 22000 \\
\hline [CW83] 0904-02 & FEROS & 074.B-0455 & 3527-9216 & 1000 & 48000 \\
\hline [CW83] 0904-02 & FEROS & 074.B-0455 & $3527-9216$ & 1000 & 48000 \\
\hline [CW83] 0904-02 & FUSE & p2051302000 & $905-1185$ & 3157 & 22000 \\
\hline [CW83] 0904-02 & FUSE & p2051303000 & $905-1185$ & 2385 & 22000 \\
\hline LSS 1274 & UVES & 077.C-0547(A) & $4726-6835$ & 2100 & 66320 \\
\hline LSS 1274 & UVES & 077.C-0547(A) & $4726-6835$ & 2100 & 66320 \\
\hline LSS 1274 & UVES & 077.C-0547(A) & $3024-3884$ & 2100 & 49620 \\
\hline LSS 1274 & UVES & 077.C-0547(A) & $3024-3884$ & 2100 & 49620 \\
\hline LSS 1274 & CASPEC & & $3925-4953$ & & 18000 \\
\hline LSS 1274 & FUSE & p2051701000 & 905-1185 & 13927 & 22000 \\
\hline LSS 1274 & FUSE & p2051702000 & $905-1185$ & 7935 & 22000 \\
\hline $\mathrm{LS}$ IV $+10^{\circ} 9$ & UVES & 67.D-0047(A) & $3281-4562$ & 1500 & 53750 \\
\hline $\mathrm{LS}$ IV $+10^{\circ} 9$ & CASPEC & & $3930-4888$ & & 18000 \\
\hline $\mathrm{LS}$ IV $+10^{\circ} 9$ & FUSE & p2050901000 & $905-1185$ & 6639 & 22000 \\
\hline $\mathrm{LS}$ IV $+10^{\circ} 9$ & FUSE & p2050903000 & $905-1185$ & 45577 & 22000 \\
\hline
\end{tabular}

Notes. We list the name of the star, the instrument used, the Program ID, the available wavelength range, the exposure time and the average resolving power for the observations we included in our analysis. For LS IV $+10^{\circ} 9$, only the blue part of the UVES spectrum was available in the ESO Data Archive.

Table A.2. Visual hydrogen and helium lines used fors the determination of atmospheric parameters.

\begin{tabular}{|c|c|}
\hline & Wavelength $(\AA)$ \\
\hline He I & 3888.65 \\
\hline He II/H I & $3968.44 / 3970.08$ \\
\hline $\mathrm{He}$ II/He I & $4025.61 / 4026.19$ \\
\hline $\mathrm{He}_{\mathrm{II}} / \mathrm{H}_{\mathrm{I}}$ & $4100.05 / 4101.71$ \\
\hline He II & 4199.84 \\
\hline He II/H I & $4338.68 / 4340.47$ \\
\hline He I & 4387.93 \\
\hline $\mathrm{He}_{\mathrm{I}}$ & 4437.55 \\
\hline $\mathrm{He}_{\mathrm{I}}$ & 4471.48 \\
\hline He II & 4541.59 \\
\hline He II & 4685.70 \\
\hline $\mathrm{He}_{\mathrm{I}}$ & 4713.15 \\
\hline $\mathrm{He}$ II/H I & $4859.32 / 4861.35$ \\
\hline $\mathrm{He}_{\mathrm{I}}$ & 4921.93 \\
\hline He I & 5015.68 \\
\hline $\mathrm{He}_{\mathrm{I}}$ & 5047.74 \\
\hline He II & 5411.51 \\
\hline He I & 5875.75 \\
\hline $\mathrm{He}_{\mathrm{II}} / \mathrm{H}_{\mathrm{I}}$ & $6560.09 / 6562.79$ \\
\hline He I & 6678.15 \\
\hline
\end{tabular}

\section{A.3. Hydrogen and helium line profile fits for [CW83] 0904-02 and LSIV $+10^{\circ} 9$}

Figure A.1 shows fits with a $\mathrm{H} / \mathrm{He} / \mathrm{C}$ grid to an UVES spectrum of [CW83] 0904-02. For details see Sect. 4.

\section{A.4. A reanalysis of CASPEC spectra}

Previous spectral analyses of our program stars (Bauer \& Husfeld 1995) and (Dreizler 1993) used atmospheric models and analysis strategies different from ours. In order to investigate the systematic differences arising, we reanalyzed the visual spectra used in the previous investigations, that is making use of the same intermediate resolution $(R=18000)$ CASPEC spectra.

In his analysis of LS IV $+10^{\circ} 9, \quad$ LSS 1274, and [CW83] 0904-02, Dreizler (1993) fixed the helium abundance at 100 times that of hydrogen to determine the effective temperature and gravity, which we adopt here, too.

For CD- $31^{\circ} 4800$ the helium abundance was fixed to $\log y=$ 2.6 as this matched the data best.

We compare our results for the atmospheric parameters obtained with the pure $\mathrm{H} / \mathrm{He}$ grid and a $\mathrm{H} / \mathrm{He}+\mathrm{X}$ grid, where $\mathrm{X}$ is the most abundant element metal (either 

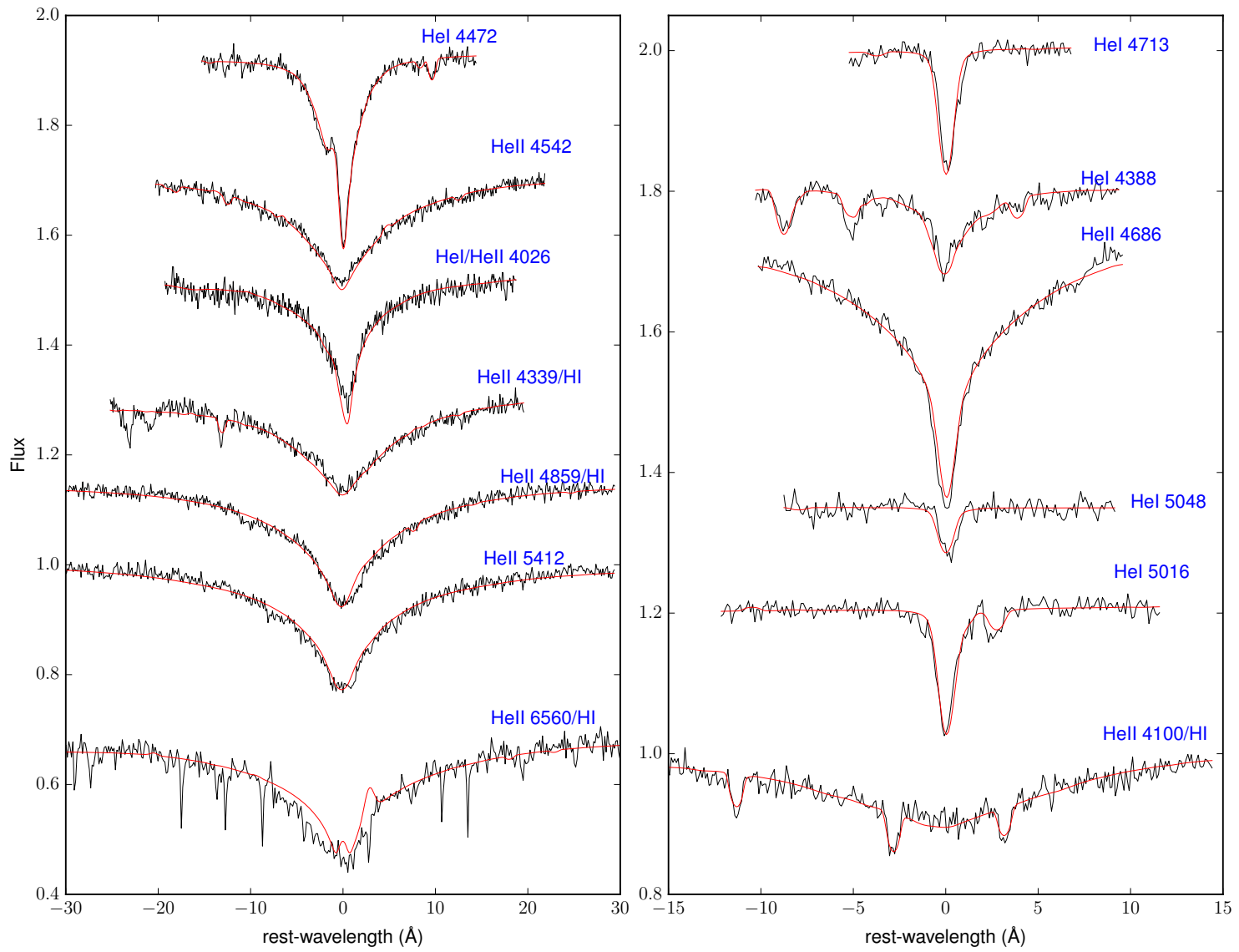

Fig. A.1. He I and He II line profiles from the final model compared to the normalized observed spectrum of [CW83] 0904-02. The spectra have been corrected for radial velocity. We note that telluric lines in the $\mathrm{He} \mathrm{I} / \mathrm{H}$, $6560 \AA$ line are particularly strong and also affect the core of that line, in particular near the predicted $\mathrm{H} \alpha$ emission component. Because it was not possible to match the He II/H 6560 line it was not included in the fit and is only shown for comparison here. The rotational velocity of [CW83] 0904-02 leads to a notable broadening and smearing of the line profiles.

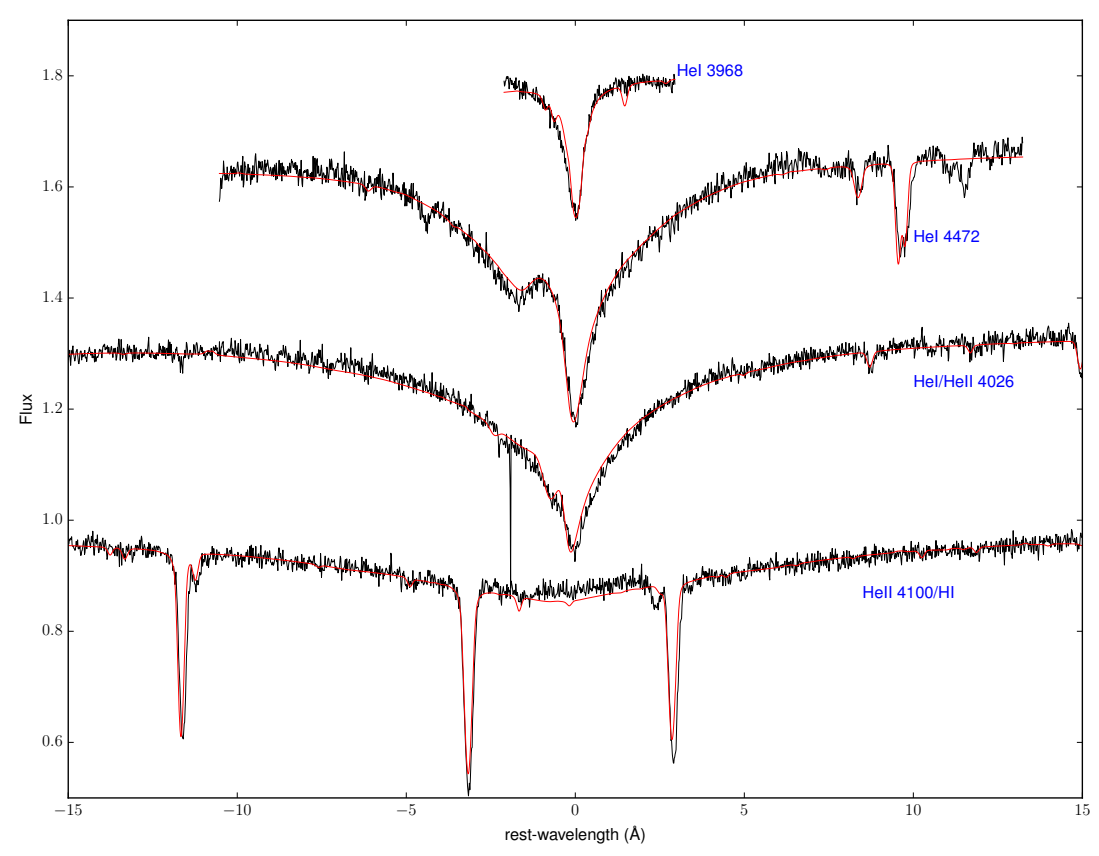

Fig. A.2. He $\mathrm{I}$ and $\mathrm{He}$ in line profiles from the final models compared to the normalized observed spectrum of LS IV $+10^{\circ}$ 9. The spectra have been corrected for radial velocity. 
Table A.3. Comparison of atmospheric parameters to literature values determined from CASPEC spectra using grids with different metallicity.

\begin{tabular}{|c|c|c|c|c|}
\hline \multirow{2}{*}{ Star } & \multirow{2}{*}{ Parameters } & $\mathrm{H} / \mathrm{He}$ & H/He (Tlusty) & $\mathrm{H} / \mathrm{He}+\mathrm{X}$ (Tlusty) \\
\hline & & $\begin{array}{c}\text { Bauer \& Husfeld (1995) } \\
\text { Dreizler (1993) }\end{array}$ & This work & This work \\
\hline \multirow{4}{*}{$\mathrm{CD}-31^{\circ} 4800$} & $T_{\text {eff }}(\mathrm{K})$ & $44000 \pm 2000$ & $42790 \pm 700$ & $42570 \pm 500$ \\
\hline & $\log g\left(\mathrm{~cm} \mathrm{~s}^{-2}\right)$ & $5.4 \pm 0.3$ & $5.41 \pm 0.08$ & $5.50 \pm 0.07$ \\
\hline & $\log y$ (fixed) & & 2.6 & 2.6 \\
\hline & $T_{\text {eff }}(\mathrm{K})$ & $44500 \pm 1000$ & $43200 \pm 600$ & $43700 \pm 500$ \\
\hline \multirow[t]{2}{*}{ LSS 1274} & $\log g\left(\mathrm{~cm} \mathrm{~s}^{-2}\right)$ & $5.55 \pm 0.15$ & $5.22 \pm 0.05$ & $5.25 \pm 0.10$ \\
\hline & $\log y$ (fixed) & 2.0 & 2.0 & 2.0 \\
\hline \multirow{3}{*}{$\mathrm{LS} \mathrm{IV}+10^{\circ} 9$} & $T_{\text {eff }}(\mathrm{K})$ & $44500 \pm 1000$ & $45880 \pm 500$ & $45420 \pm 750$ \\
\hline & $\log g\left(\mathrm{~cm} \mathrm{~s}^{-2}\right)$ & $5.55 \pm 0.15$ & $5.48 \pm 0.05$ & $5.58 \pm 0.08$ \\
\hline & $\log y$ (fixed) & 2.0 & 2.0 & 2.0 \\
\hline \multirow{3}{*}{ [CW83] 0904-02 } & $T_{\text {eff }}(\mathrm{K})$ & $46500 \pm 1000$ & $48090 \pm 800$ & $48090 \pm 750$ \\
\hline & $\log g\left(\mathrm{~cm} \mathrm{~s}^{-2}\right)$ & $5.55 \pm 0.15$ & $5.40 \pm 0.07$ & $5.49 \pm 0.07$ \\
\hline & $\log y$ (fixed) & 2.0 & 2.0 & 2.0 \\
\hline
\end{tabular}

nitrogen for $\mathrm{CD}-31^{\circ} 4800$ or carbon for the other stars) in Table A.3.

From Table A.3 it is apparent that the results from our $\mathrm{H} / \mathrm{He}$ grid are all in accordance with the results from previous investigations and the error ranges overlap for all parameters.

The effects of the additional metals in the $\mathrm{H} / \mathrm{He}+\mathrm{C}$ and $\mathrm{H} / \mathrm{He}+\mathrm{N}$ grids can be explained by the effects of back-warming. The atmospheric parameters derived from the analyses of CASPEC spectra and those derived from of modern visual spectra agree also very well (cf. Table 4).

\section{A.5. The $C_{\mathrm{II}}, 4267 \AA$ line}

The spectral line of singly ionized carbon $\left(\mathrm{C}_{\text {II }}\right)$ at $4267 \AA$ is present in the visual spectra of the three C-rich stars (see Sects. 7.1.1), but is very weak. Nevertheless, lines of ionized carbon were included in models containing $\mathrm{H}, \mathrm{He}, \mathrm{C}, \mathrm{N}$, and $\mathrm{O}$, but had to be excluded in the full model, because the very low population of $\mathrm{C}$ II atomic levels led to numerical instabilities and prevented the models to converge. However, the carbon abundance determined in the latter model (from $\mathrm{C}_{\text {III }}$ and C IV lines only) differs from the one of the former model ( $\mathrm{C}$ II, $\mathrm{C}$ III, and $\mathrm{C}_{\text {IV }}$ ) by $0.02 \mathrm{dex}$, only, which is much less than the abundance uncertainties and, therefore, insignificant. In order to evaluate $C_{\text {II }}, 4267 \AA$ (see Sect. 7.1.1) line profiles from the final models and adopted $\mathrm{C}$ abundances (Tables 7 and 8) are compared to the observed spectra in Fig. A.3. For CD$31^{\circ} 4800$ the line is predicted to be too weak to be observable, consistent with observations. The line is hardly visible in the spectrum of [CW83] 0904-02 because of the star's rotation. For LS IV $+10^{\circ} 9$ the observed profile is well reproduced, while the line is predicted stronger than observed for LSS 1274.

A fit to match the individual line (see Fig. A.3) results in $\log \left(n_{\mathrm{C}} / n_{\mathrm{H}}\right)=0.11 \pm 0.10$ for $\mathrm{LSIV}+10^{\circ} 9$ and $\log \left(n_{\mathrm{C}} / n_{\mathrm{H}}\right)=-0.35 \pm 0.09$ for LSS 1274, which are close to the adopted $\mathrm{C} / \mathrm{H}$ ratios of $0.15 \mathrm{dex}$ and $-0.01 \mathrm{dex}$, respectively, to within the scatter range of individual $\mathrm{C}_{\text {III }}$ and $\mathrm{C}_{\mathrm{IV}}$ lines.

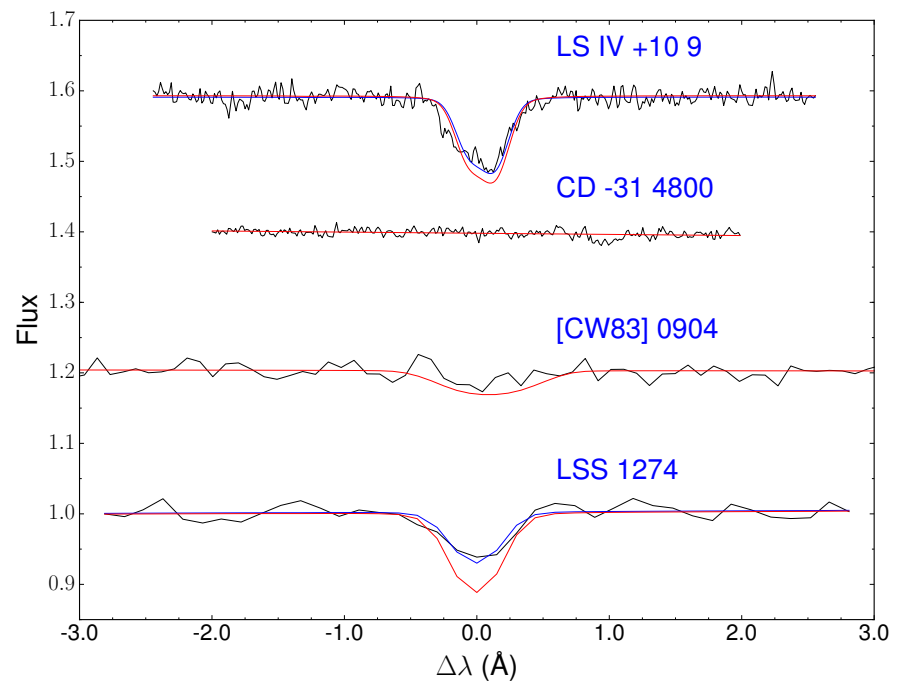

Fig. A.3. Comparison of the observed $C_{\text {II }}, 4267 \AA$ line to the prediction from the final model. Synthetic line profiles calculated for the $\mathrm{C}$ abundances listed in Tables 7 and 8 are plotted in red. For LS IV $+10^{\circ} 9$ and LSS 1274 the blue profiles represent the best fits to the line.

\section{A.6. SED and photometric fit for $C D-31^{\circ} 4800, L S I V+10^{\circ} 9$, and [CW83] 0904-02}

Figure A.4 shows the fits to the spectral energy distribution of CD-31 ${ }^{\circ} 4800, \mathrm{LS} \mathrm{IV}+10^{\circ} 9$, and [CW83] 0904-02 (from top to bottom). Overlayed on the SED (gray) are the measurements for different magnitudes in different systems. The residuals for the magnitudes and colors are given in the smaller panels below and to the right of the SED. For details see Sect. 6.

\section{A.7. Line list for abundance analysis}

In Tables A.4 and A.5 we list the spectral lines used for the metal abundance analysis for the visual/UVA spectral and the FUV spectral range respectively. 

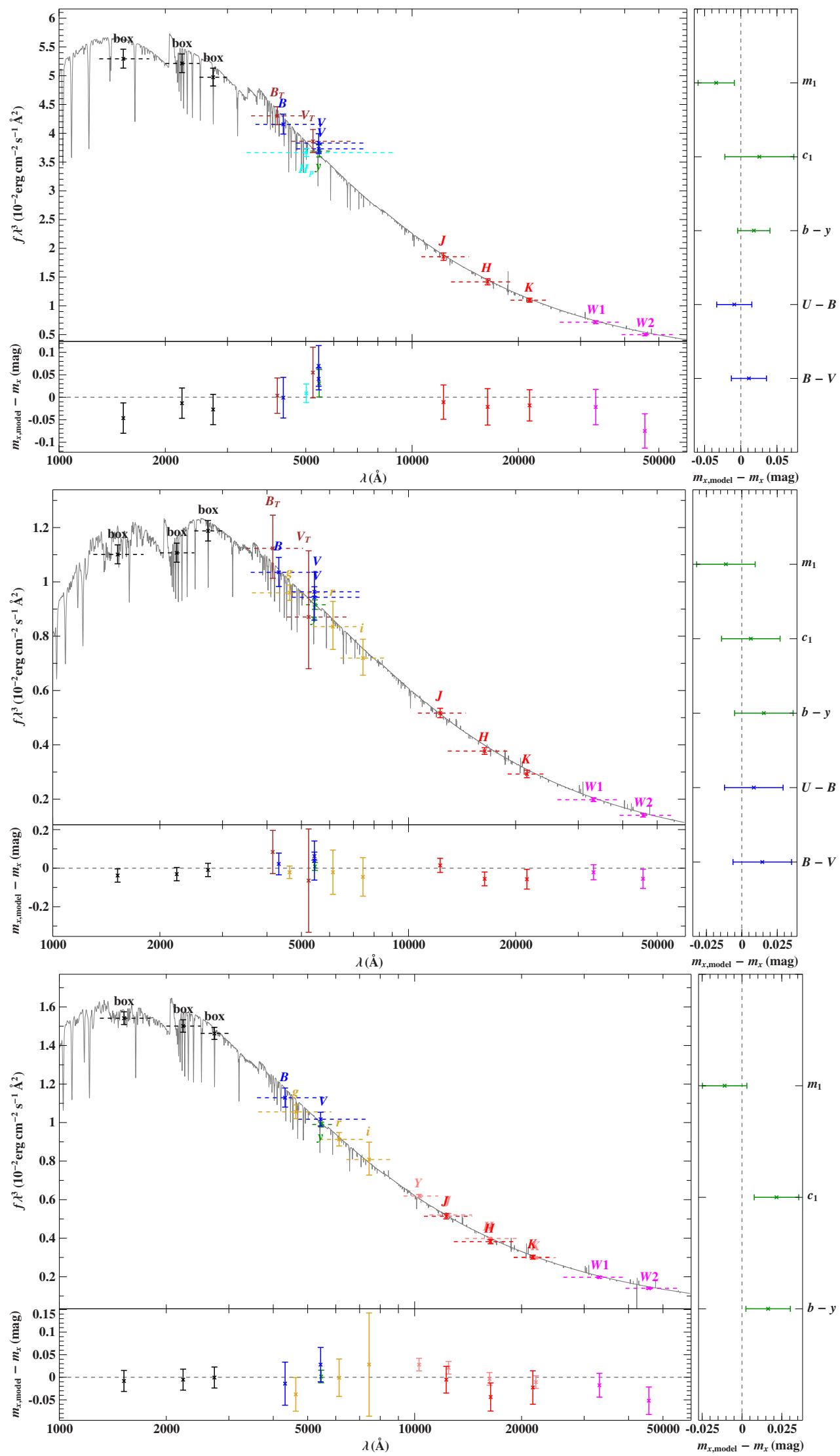

Fig. A.4. Matching the spectral energy distributions and colors of CD-31 4800 (upper panel), LS IV $+10^{\circ} 9$ (middle panel), and [CW83] 0904-02 (lower panel). The synthetic SEDs (grey) are overlayed to the fluxes derived from observed apparent magnitudes in different photometric systems. The residuals for the magnitudes and colors are given in the panels below (SED) and to the right (colors) of the main panel. 
Table A.4. Visual and UVA spectral lines used for the abundance determination.

\begin{tabular}{|c|c|}
\hline Element+ion. stage & Wavelength $(\AA)$ \\
\hline $\mathrm{C}_{\text {III }}$ & 3883.81 \\
\hline $\mathrm{C}_{\text {III }}$ & $*$ \\
\hline$*$ & 3608.78 \\
\hline$*$ & 3609.05 \\
\hline$*$ & 3609.07 \\
\hline$*$ & 3609.62 \\
\hline$*$ & 3609.68 \\
\hline $\mathrm{C}_{\text {III }}$ & 4056.06 \\
\hline $\mathrm{C}_{\text {III }}$ & $*$ \\
\hline$*$ & 4067.94 \\
\hline$*$ & 4068.91 \\
\hline$*$ & 4070.26 \\
\hline $\mathrm{C}_{\text {III }}$ & 4121.85 \\
\hline $\mathrm{C}_{\text {III }}$ & 4186.90 \\
\hline $\mathrm{C}_{\text {III }}$ & 4247.31 \\
\hline $\mathrm{C}_{\text {III }}$ & 4325.56 \\
\hline $\mathrm{C}_{\text {III }}$ & 4361.85 \\
\hline $\mathrm{C}_{\text {III }}$ & 4382.90 \\
\hline $\mathrm{C}_{\text {III }}$ & 4516.79 \\
\hline $\mathrm{C}_{\text {III }}$ & $*$ \\
\hline$*$ & 4647.42 \\
\hline$*$ & 4650.25 \\
\hline$*$ & 4651.02 \\
\hline$*$ & 4651.47 \\
\hline$*$ & 4652.05 \\
\hline $\mathrm{C}_{\text {III }}$ & 5695.92 \\
\hline $\mathrm{C}_{\text {III }}$ & $*$ \\
\hline$*$ & 6149.28 \\
\hline$*$ & 6154.16 \\
\hline$*$ & 6155.12 \\
\hline$*$ & 6156.69 \\
\hline$*$ & 6160.01 \\
\hline C IV & 4658.30 \\
\hline C IV & * \\
\hline$*$ & 5016.62 \\
\hline$*$ & 5018.40 \\
\hline CIV & $*$ \\
\hline$*$ & 5801.33 \\
\hline$*$ & 5811.97 \\
\hline $\mathrm{N}_{\text {II }}$ & 3838.37 \\
\hline $\mathrm{N}_{\text {II }}$ & 3842.19 \\
\hline $\mathrm{N}_{\text {II }}$ & 3995.00 \\
\hline $\mathrm{N}_{\text {II }}$ & 4176.16 \\
\hline $\mathrm{N}_{\text {II }}$ & 4227.74 \\
\hline $\mathrm{N}_{\text {II }}$ & 4447.03 \\
\hline $\mathrm{N}_{\text {II }}$ & 4694.27 \\
\hline $\mathrm{N}_{\text {II }}$ & 4779.72 \\
\hline $\mathrm{N}_{\text {II }}$ & 4788.13 \\
\hline $\mathrm{N}_{\text {II }}$ & 4803.29 \\
\hline $\mathrm{N}_{\text {II }}$ & 4987.38 \\
\hline $\mathrm{N}_{\text {II }}$ & 5002.80 \\
\hline $\mathrm{N}_{\text {II }}$ & 5007.32 \\
\hline
\end{tabular}

Notes. The profiles of some spectral lines were calculated together, either because they are closely blended lines or resolved multiple lines, for which no lines from other species intervene (e.g. Ne II, 3643.93/3644.86 ̊, see Fig. 6). The wavelengths of such "combined" lines are marked by asterisks.
Table A.4. Continued.

\begin{tabular}{|c|c|}
\hline Element+ion. stage & Wavelength $(\AA)$ \\
\hline $\mathrm{N}_{\text {II }}$ & 5010.62 \\
\hline $\mathrm{N}_{\text {II }}$ & 5025.66 \\
\hline $\mathrm{N}_{\text {II }}$ & 5045.10 \\
\hline $\mathrm{N}_{\text {II }}$ & 5495.67 \\
\hline $\mathrm{N}_{\text {II }}$ & 5666.63 \\
\hline $\mathrm{N}_{\text {II }}$ & 5676.02 \\
\hline $\mathrm{N}_{\text {II }}$ & 5679.56 \\
\hline $\mathrm{N}_{\text {II }}$ & 5686.21 \\
\hline $\mathrm{N}_{\text {II }}$ & 5710.77 \\
\hline $\mathrm{N}_{\text {II }}$ & 5931.78 \\
\hline $\mathrm{N}_{\text {III }}$ & 3004.03 \\
\hline $\mathrm{N}_{\text {III }}$ & 3330.11 \\
\hline $\mathrm{N}_{\text {III }}$ & 3342.09 \\
\hline $\mathrm{N}_{\text {III }}$ & $*$ \\
\hline$*$ & 3353.98 \\
\hline$*$ & 3354.32 \\
\hline $\mathrm{N}_{\text {III }}$ & $*$ \\
\hline$*$ & 3360.98 \\
\hline$*$ & 3365.80 \\
\hline $\mathrm{N}_{\text {III }}$ & $*$ \\
\hline$*$ & 3365.80 \\
\hline$*$ & 3367.30 \\
\hline$*$ & 3374.07 \\
\hline $\mathrm{N}_{\text {III }}$ & $*$ \\
\hline$*$ & 3754.62 \\
\hline$*$ & 3757.65 \\
\hline$*$ & 3762.60 \\
\hline $\mathrm{N}_{\text {III }}$ & $*$ \\
\hline$*$ & 3771.05 \\
\hline$*$ & 3771.36 \\
\hline $\mathrm{N}_{\text {III }}$ & 3792.97 \\
\hline $\mathrm{N}_{\text {III }}$ & 3938.52 \\
\hline $\mathrm{N}_{\text {III }}$ & 3942.88 \\
\hline $\mathrm{N}_{\text {III }}$ & $*$ \\
\hline$*$ & 3998.63 \\
\hline$*$ & 4003.58 \\
\hline $\mathrm{N}_{\text {III }}$ & 4097.33 \\
\hline $\mathrm{N}_{\text {III }}$ & 4103.39 \\
\hline $\mathrm{N}_{\text {III }}$ & 4195.70 \\
\hline $\mathrm{N}_{\text {III }}$ & 4200.02 \\
\hline $\mathrm{N}_{\text {III }}$ & 4215.77 \\
\hline $\mathrm{N}_{\text {III }}$ & 4318.78 \\
\hline $\mathrm{N}_{\text {III }}$ & $*$ \\
\hline$*$ & 4318.78 \\
\hline$*$ & 4321.22 \\
\hline$*$ & 4325.43 \\
\hline$*$ & 4327.88 \\
\hline $\mathrm{N}_{\text {III }}$ & * \\
\hline$*$ & 4332.91 \\
\hline$*$ & 4337.01 \\
\hline $\mathrm{N}_{\text {III }}$ & $*$ \\
\hline$*$ & 4345.68 \\
\hline$*$ & 4345.81 \\
\hline$*$ & 4351.11 \\
\hline $\mathrm{N}_{\text {III }}$ & $*$ \\
\hline$*$ & 4514.86 \\
\hline$*$ & 4518.91 \\
\hline $\mathrm{N}_{\text {III }}$ & 4523.56 \\
\hline $\mathrm{N}_{\text {III }}$ & 4530.86 \\
\hline
\end{tabular}


Table A.4. Continued.

\begin{tabular}{|c|c|}
\hline Element+ion. stage & Wavelength $(\AA)$ \\
\hline $\mathrm{N}_{\text {III }}$ & 4534.58 \\
\hline $\mathrm{N}_{\text {III }}$ & 4547.30 \\
\hline $\mathrm{N}_{\text {III }}$ & $*$ \\
\hline$*$ & 4589.18 \\
\hline$*$ & 4591.98 \\
\hline $\mathrm{N}_{\text {III }}$ & 4634.14 \\
\hline $\mathrm{N}$ III $+\mathrm{He}_{\text {II }} \& \mathrm{H} \beta$ & $*$ \\
\hline$*$ & 4858.82 \\
\hline$*$ & 4861.22 \\
\hline$*$ & 4867.15 \\
\hline $\mathrm{N}_{\text {III }}$ & 4873.10 \\
\hline $\mathrm{N}_{\text {III }}$ & 4884.14 \\
\hline $\mathrm{N}_{\text {III }}$ & $*$ \\
\hline$*$ & 5260.86 \\
\hline$*$ & 5270.57 \\
\hline$*$ & 5272.68 \\
\hline$*$ & 5282.43 \\
\hline N III & * \\
\hline$*$ & 5297.75 \\
\hline$*$ & 5298.95 \\
\hline \multicolumn{2}{|l|}{$\mathrm{N}_{\text {III }}^{*}$} \\
\hline$*$ & 5314.35 \\
\hline$*$ & 5320.32 \\
\hline$*$ & 5327.18 \\
\hline $\mathrm{N}_{\text {III }}$ & $*$ \\
\hline$*$ & 5817.79 \\
\hline$*$ & 5820.57 \\
\hline N III & 5847.94 \\
\hline $\mathrm{N}_{\text {III }}$ & 5954.70 \\
\hline Niv & $*$ \\
\hline$*$ & 3478.71 \\
\hline$*$ & 3482.99 \\
\hline$*$ & 3484.96 \\
\hline $\mathrm{N}_{\text {IV }}$ & 4057.76 \\
\hline N IV & 6380.77 \\
\hline $\mathrm{O}_{\text {II }}$ & 3759.87 \\
\hline $\mathrm{O}_{\text {II }}$ & 3857.16 \\
\hline $\mathrm{O}_{\text {II }}$ & 4069.89 \\
\hline $\mathrm{O}_{\text {II }}$ & 4072.15 \\
\hline $\mathrm{O}_{\text {II }}$ & 4075.86 \\
\hline $\mathrm{O}_{\text {II }}$ & $*$ \\
\hline$*$ & 4307.23 \\
\hline$*$ & 4312.10 \\
\hline$*$ & 4313.44 \\
\hline$*$ & 4317.14 \\
\hline$*$ & 4317.70 \\
\hline$*$ & 4319.63 \\
\hline$*$ & 4325.75 \\
\hline $\mathrm{O}_{\text {II }}$ & 4349.42 \\
\hline $\mathrm{O}_{\mathrm{II}}$ & 4336.87 \\
\hline $\mathrm{O}_{\mathrm{II}}$ & 6152.56 \\
\hline O III & 3340.74 \\
\hline $\mathrm{O}_{\text {III }}$ & 3459.48 \\
\hline O III & 3715.08 \\
\hline O III & 3757.21 \\
\hline $\mathrm{O}_{\text {III }}$ & 3759.87 \\
\hline $\mathrm{O}_{\text {III }}$ & 5592.37 \\
\hline $\mathrm{O}_{\text {III }}$ & 6507.55 \\
\hline $\mathrm{Ne}$ II & 3323.73 \\
\hline
\end{tabular}

Table A.4. Continued.

\begin{tabular}{|c|c|}
\hline Element+ion. stage & Wavelength $(\AA)$ \\
\hline $\mathrm{Ne}$ II & 3327.15 \\
\hline $\mathrm{Ne}$ II & 3388.41 \\
\hline $\mathrm{Ne}$ II & 3406.94 \\
\hline $\mathrm{Ne}$ II & $*$ \\
\hline$*$ & 3416.91 \\
\hline$*$ & 3417.69 \\
\hline $\mathrm{Ne}$ II & 3542.84 \\
\hline $\mathrm{Ne}_{\text {II }}$ & $*$ \\
\hline$*$ & 3565.82 \\
\hline$*$ & 3568.50 \\
\hline$*$ & 3571.23 \\
\hline$*$ & 3574.61 \\
\hline $\mathrm{Ne}$ II & $*$ \\
\hline$*$ & 3643.93 \\
\hline$*$ & 3644.86 \\
\hline $\mathrm{Ne}$ II & 3664.07 \\
\hline $\mathrm{Ne}$ II & 3694.21 \\
\hline $\mathrm{Ne}$ II & 3709.62 \\
\hline $\mathrm{Ne}$ II & 3713.08 \\
\hline $\mathrm{Ne}$ II & 3727.11 \\
\hline $\mathrm{Ne}$ II & 3766.26 \\
\hline $\mathrm{Ne}$ II & 3777.13 \\
\hline $\mathrm{Ne}$ II & 4150.69 \\
\hline $\mathrm{Ne}$ II & $*$ \\
\hline$*$ & 4217.17 \\
\hline$*$ & 4219.37 \\
\hline$*$ & 4219.74 \\
\hline $\mathrm{Ne}$ II & 4250.64 \\
\hline $\mathrm{Ne}$ II & $*$ \\
\hline$*$ & 4290.37 \\
\hline$*$ & 4290.60 \\
\hline $\mathrm{Ne}$ II & 4379.55 \\
\hline $\mathrm{Ne}$ II & 4397.99 \\
\hline $\mathrm{Ne}$ II & $*$ \\
\hline$*$ & 4409.30 \\
\hline$*$ & 4412.59 \\
\hline $\mathrm{Ne}$ II & $*$ \\
\hline$*$ & 4428.52 \\
\hline$*$ & 4428.63 \\
\hline$*$ & 4430.90 \\
\hline$*$ & 4431.81 \\
\hline $\mathrm{Mg}_{\text {II }}$ & 4242.45 \\
\hline $\mathrm{Mg}_{\text {II }}$ & $*$ \\
\hline$*$ & 4481.13 \\
\hline$*$ & 4481.33 \\
\hline $\mathrm{Mg}_{\text {II }}$ & $*$ \\
\hline$*$ & 5916.43 \\
\hline$*$ & 5918.16 \\
\hline $\mathrm{Mg}_{\text {II }}$ & 5928.23 \\
\hline $\mathrm{Mg}_{\text {II }}$ & $*$ \\
\hline$*$ & 6346.74 \\
\hline$*$ & 6346.96 \\
\hline Al III & 3601.63 \\
\hline $\mathrm{Al}$ III & 4149.96 \\
\hline$*$ & 4159.91 \\
\hline$*$ & 4149.96 \\
\hline$*$ & 4150.17 \\
\hline Al III & $*$ \\
\hline
\end{tabular}


M. Schindewolf et al.: A quantitative NLTE analysis of four helium-rich subdwarf O stars

Table A.4. Continued.

\begin{tabular}{|c|c|}
\hline Element+ion. stage & Wavelength $(\AA)$ \\
\hline$*$ & 4479.83 \\
\hline$*$ & 4479.97 \\
\hline Al III & 4512.57 \\
\hline Al III & 5722.73 \\
\hline Al III & 5696.60 \\
\hline $\mathrm{Si}$ III & 3486.91 \\
\hline Si III & 3791.41 \\
\hline Si III & 3806.54 \\
\hline Si III & 3924.47 \\
\hline Si III & 4552.65 \\
\hline Si III & 4567.87 \\
\hline $\mathrm{Si}$ III & 4574.78 \\
\hline Si III & 4716.66 \\
\hline $\mathrm{Si}$ III & 4813.39 \\
\hline Si III & 4819.72 \\
\hline Si III & 5739.73 \\
\hline Si IV & 3773.15 \\
\hline Si IV & 4088.86 \\
\hline Si IV & 4116.10 \\
\hline Si IV & 4212.41 \\
\hline Si IV & 4314.10 \\
\hline Si IV & 4631.24 \\
\hline Si IV & 4654.32 \\
\hline Si IV & 4950.11 \\
\hline P IV & 3347.74 \\
\hline P IV & 3364.47 \\
\hline P IV & 4249.66 \\
\hline PIV & 4728.37 \\
\hline $\mathrm{Pv}_{\mathrm{v}}$ & 5122.19 \\
\hline $\mathrm{S}_{\text {III }}$ & 3632.02 \\
\hline$S_{\text {III }}$ & 3662.01 \\
\hline
\end{tabular}

Table A.5. UV lines in the FUSE spectra used for the abundance determination.

\begin{tabular}{|c|c|}
\hline Element+ion. stage & Wavelength $(\AA)$ \\
\hline $\mathrm{S}_{\text {III }}$ & 3717.77 \\
\hline S III & 3747.85 \\
\hline $\mathrm{S}_{\text {III }}$ & 3794.68 \\
\hline $\mathrm{S}_{\text {III }}$ & 3837.79 \\
\hline S III & 3928.61 \\
\hline S III & 3961.51 \\
\hline S III & 4099.15 \\
\hline$S_{\text {III }}$ & 4253.59 \\
\hline S III & 4284.99 \\
\hline $\mathrm{S}_{\text {III }}$ & 4361.47 \\
\hline S IV & 5488.25 \\
\hline S IV & 5497.75 \\
\hline $\mathrm{C}_{\text {III }}$ & 1051.15 \\
\hline $\mathrm{C}_{\text {III }}$ & $*$ \\
\hline$*$ & 1070.17 \\
\hline$*$ & 1070.33 \\
\hline $\mathrm{C}_{\text {III }}$ & $*$ \\
\hline$*$ & 1125.63 \\
\hline$*$ & 1125.64 \\
\hline$*$ & 1125.65 \\
\hline$*$ & 1125.67 \\
\hline
\end{tabular}

Table A.5. Continued.

\begin{tabular}{|c|c|}
\hline Element + ion. stage & Wavelength $(\AA)$ \\
\hline$*$ & 1125.68 \\
\hline $\mathrm{C}_{\text {III }}$ & 1139.90 \\
\hline C III & $*$ \\
\hline$*$ & 1148.89 \\
\hline$*$ & 1148.90 \\
\hline$*$ & 1148.91 \\
\hline$*$ & 1148.92 \\
\hline$*$ & 1148.94 \\
\hline $\mathrm{C}_{\text {III }}$ & $*$ \\
\hline$*$ & 1165.62 \\
\hline$*$ & 1165.70 \\
\hline $\mathrm{C}_{\text {III }}$ & $*$ \\
\hline$*$ & 1174.93 \\
\hline$*$ & 1175.26 \\
\hline$*$ & 1175.59 \\
\hline$*$ & 1175.71 \\
\hline$*$ & 1175.99 \\
\hline$*$ & 1176.37 \\
\hline $\mathrm{C}_{\mathrm{IV}}$ & 1107.98 \\
\hline $\mathrm{N}_{\text {III }}$ & $*$ \\
\hline$*$ & 1005.99 \\
\hline$*$ & 1006.04 \\
\hline $\mathrm{N}_{\text {III }}$ & * \\
\hline$*$ & 1038.61 \\
\hline$*$ & 1038.98 \\
\hline$*$ & 1039.00 \\
\hline $\mathrm{N}_{\text {III }}$ & $*$ \\
\hline$*$ & 1055.95 \\
\hline$*$ & 1055.97 \\
\hline $\mathrm{N}_{\text {III }}$ & 1103.05 \\
\hline $\mathrm{N}_{\text {IIII }}+\mathrm{N}_{\text {IV }}$ & * \\
\hline$*$ & 1104.07 \\
\hline$*$ & 1104.12 \\
\hline$*$ & 1104.54 \\
\hline $\mathrm{N}_{\text {III }}$ & 1106.34 \\
\hline $\mathrm{N}_{\text {III }}$ & $*$ \\
\hline$*$ & 1120.60 \\
\hline$*$ & 1120.81 \\
\hline $\mathrm{N}_{\text {III }}$ & $*$ \\
\hline$*$ & 1140.05 \\
\hline$*$ & 1140.12 \\
\hline \multicolumn{2}{|l|}{$\mathrm{N}_{\text {III }}$} \\
\hline$*$ & 1182.97 \\
\hline$*$ & 1183.03 \\
\hline $\mathrm{N}_{\text {III }}$ & $*$ \\
\hline$*$ & 1184.51 \\
\hline$*$ & 1184.55 \\
\hline N IV & 955.34 \\
\hline $\mathrm{N}_{\text {IV }}$ & $*$ \\
\hline$*$ & 1036.12 \\
\hline$*$ & 1036.15 \\
\hline$*$ & 1036.20 \\
\hline$*$ & 1036.23 \\
\hline$*$ & 1036.24 \\
\hline$*$ & 1036.33 \\
\hline N IV & 1117.93 \\
\hline N IV & $*$ \\
\hline$*$ & 1132.02 \\
\hline$*$ & 1132.23 \\
\hline
\end{tabular}


Table A.5. Continued.

\begin{tabular}{|c|c|}
\hline Element+ion. stage & Wavelength $(\AA)$ \\
\hline $\mathrm{N}_{\text {IV }}$ & * \\
\hline$*$ & 1132.68 \\
\hline$*$ & 1132.94 \\
\hline$*$ & 1133.12 \\
\hline $\mathrm{N}_{\text {IV }}$ & 1135.25 \\
\hline Niv & 1188.01 \\
\hline $\mathrm{O}_{\text {III }}$ & * \\
\hline$*$ & 1007.88 \\
\hline$*$ & 1008.10 \\
\hline$*$ & 1008.40 \\
\hline O III & 1033.15 \\
\hline $\mathrm{O}_{\text {III }}$ & 1040.32 \\
\hline O III & 1138.54 \\
\hline $\mathrm{O}_{\text {III }}$ & $*$ \\
\hline$*$ & 1150.88 \\
\hline$*$ & 1150.99 \\
\hline $\mathrm{O}_{\text {III }}$ & * \\
\hline$*$ & 1153.02 \\
\hline$*$ & 1153.21 \\
\hline O III & 1153.77 \\
\hline $\mathrm{O}_{\text {III }}$ & 1157.64 \\
\hline O IV & 1106.93 \\
\hline O IV & 1164.55 \\
\hline $\mathrm{Ne}$ II & 1133.92 \\
\hline $\mathrm{Ne}_{\text {II }}$ & $*$ \\
\hline$*$ & 1139.11 \\
\hline$*$ & 1139.36 \\
\hline $\mathrm{Ne}$ II & 1143.89 \\
\hline $\mathrm{Ne}$ III & $*$ \\
\hline$*$ & 1044.94 \\
\hline$*$ & 1044.96 \\
\hline $\mathrm{Ne}$ III & $*$ \\
\hline$*$ & 1076.71 \\
\hline$*$ & 1076.79 \\
\hline$*$ & 1077.02 \\
\hline Al III & 1071.74 \\
\hline Al III & * \\
\hline$*$ & 1162.59 \\
\hline$*$ & 1162.62 \\
\hline Si III & 997.39 \\
\hline Si III & $*$ \\
\hline$*$ & 1113.17 \\
\hline$*$ & 1113.20 \\
\hline$*$ & 1113.23 \\
\hline Si III & 1140.50 \\
\hline Si III & 1144.31 \\
\hline Si III & 1161.58 \\
\hline Si IV & 996.87 \\
\hline Si IV & * \\
\hline$*$ & 1154.62 \\
\hline$*$ & 1154.62 \\
\hline PIV & $*$ \\
\hline$*$ & 1030.51 \\
\hline$*$ & 1030.52 \\
\hline PIV & 1033.10 \\
\hline PIV & 1064.61 \\
\hline
\end{tabular}

Table A.5. Continued.

\begin{tabular}{|c|c|}
\hline Element+ion. stage & Wavelength $(\AA)$ \\
\hline PIV & 1118.55 \\
\hline$P_{\text {IV }}$ & 1187.57 \\
\hline $\mathrm{Pv}$ & 997.60 \\
\hline $\mathrm{P}_{\mathrm{V}}$ & 1117.98 \\
\hline $\mathrm{Pv}_{\mathrm{V}}$ & 1128.01 \\
\hline $\mathrm{S}_{\text {IV }}$ & 1072.97 \\
\hline S IV & 1073.52 \\
\hline $\mathrm{S}_{\text {IV }}$ & 1098.36 \\
\hline S IV & 1098.92 \\
\hline $\mathrm{S} \mathrm{v}$ & 1122.04 \\
\hline S VI & 933.38 \\
\hline S VI & 944.53 \\
\hline $\mathrm{Fe}$ III & 1141.27 \\
\hline $\mathrm{Fe}$ III & 1142.96 \\
\hline Fe IV & 1156.53 \\
\hline $\mathrm{Fe} \mathrm{IV}+2 \mathrm{Fe} \mathrm{v}$ & 1112.18 \\
\hline$*$ & 1111.89 \\
\hline$*$ & 1112.17 \\
\hline$*$ & 1112.21 \\
\hline $\mathrm{Fe} v$ & $*$ \\
\hline$*$ & 1116.05 \\
\hline * & 1116.16 \\
\hline $\mathrm{Fe} \mathrm{IV}+\mathrm{Fe} \mathrm{v}$ & $*$ \\
\hline$*$ & 1124.83 \\
\hline$*$ & 1124.89 \\
\hline $\mathrm{Fe} v$ & 1118.38 \\
\hline $\mathrm{Fe} \mathrm{v}$ & 1133.57 \\
\hline $\mathrm{Fe} v$ & 1149.12 \\
\hline $\mathrm{Fe} v$ & 1153.80 \\
\hline $\mathrm{Fe} \mathrm{v}$ & 1161.92 \\
\hline $\mathrm{Fe} v$ & 1165.71 \\
\hline Ni IV & 1126.33 \\
\hline $\mathrm{Ni}$ IV+Ni v & 1152.68 \\
\hline$*$ & 1152.67 \\
\hline$*$ & 1152.68 \\
\hline $\mathrm{Ni}$ IV $+4 \mathrm{xNi} \mathrm{V}$ & $*$ \\
\hline$*$ & 1158.86 \\
\hline$*$ & 1158.98 \\
\hline$*$ & 1158.02 \\
\hline$*$ & 1159.04 \\
\hline$*$ & 1159.09 \\
\hline $\mathrm{Ni}$ IV $+2 x \mathrm{Ni} \mathrm{V}$ & * \\
\hline$*$ & 1187.84 \\
\hline$*$ & 1187.67 \\
\hline$*$ & 1187.79 \\
\hline $\mathrm{Niv}$ & $*$ \\
\hline$*$ & 1178.80 \\
\hline$*$ & 1178.81 \\
\hline$*$ & 1178.82 \\
\hline$*$ & 1178.92 \\
\hline$*$ & 1178.94 \\
\hline$*$ & 1179.16 \\
\hline $\mathrm{Ni} \mathrm{v}$ & * \\
\hline$*$ & 1182.54 \\
\hline$*$ & 1182.62 \\
\hline$*$ & 1182.71 \\
\hline
\end{tabular}

